\title{
Angewandte
}

Supporting Information

(c) Wiley-VCH 2013

69451 Weinheim, Germany

\section{Preparation of the Elusive [(por)Fe(NO)(O-ligand)] Complex by Diffusion of Nitric Oxide into a Crystal of the Precursor**}

Nan Xu,* Lauren E. Goodrich, Nicolai Lehnert,* Douglas R. Powell, and George B. RichterAddo*

anie_201208063_sm_miscellaneous_information.pdf 
Part A: Density Functional Theory (DFT) calculations p S2 (Methodology, Tables S1-S4)

Part B: X-ray Crystallographic Determination of $(\mathrm{TPP}) \mathrm{Fe}\left(\mathrm{OC}(=\mathrm{O}) \mathrm{CF}_{3}\right)(\mathbf{1}) \quad$ p S5 (Experimental, Figures S1-S3, Tables S5-S10)

Part C: X-ray Crystallographic Determination of (TPP)Fe(NO) $\left(\mathrm{OC}(=\mathrm{O}) \mathrm{CF}_{3}\right)(2)$ p S29 (Experimental, Figures S4-S5, Tables S11-S16) 


\section{PART A: Density Functional Theory (DFT) Calculations}

All geometry optimizations and frequency calculations were performed with the program package Gaussian $03^{1}$ using the $\mathrm{BP}^{2-3} 6^{2-3}$ functional and $\mathrm{TZVP}^{4-5}$ basis set. In Gaussian calculations, convergence was reached when the relative change in the density matrix between subsequent iterations was less that $1 \times 10^{-8}$. Molecular orbitals were obtained from BP86/TZVP single point calculations using ORCA. ${ }^{6}$ Molecular orbitals were plotted with the program orca_plot included in the ORCA package and visualized using GaussView. Force constants in internal coordinates were extracted from the Gaussian output using a modified version of the program Redong (QCPE 628). ${ }^{7-8}$

\section{References}

1. Frisch, M. J.; Trucks, G. W.; Schlegel, H. B.; Scuseria, G. E.; Robb, M. A.; Cheeseman, J. R.; Montgomery, J., J. A.; Vreven, T.; Kudin, K. N.; Burant, J. C.; Millam, J. M.; Iyengar, S. S.; Tomasi, J.; Barone, V.; Mennucci, B.; Cossi, M.; Scalmani, G.; Rega, N.; Petersson, G. A.; Nakatsuji, H.; Hada, M.; Ehara, M.; Toyota, K.; Fukuda, R.; Hasegawa, J.; Ishida, M.; Nakajima, T.; Honda, Y.; Kitao, O.; Nakai, H.; Klene, M.; Li, X.; Knox, J. E.; Hratchian, H. P.; Cross, J. B.; Bakken, V.; Adamo, C.; Jaramillo, J.; Gomperts, R.; Stratmann, R. E.; Yazyev, O.; Ausin, A. J.; Cammi, R.; Pomelli, C.; Octerski, J. W.; Ayala, P. Y.; Morokuma, K.; Voth, G. A.; Salvador, P.; Dannenberg, J. J.; Zakrzewski, V. G.; Dapprich, S.; Daniels, A. D.; Strain, M. C.; Farkas, O.; Makick, D. K.; Rabuck, A. D.; Raghavachair, K.; Foresman, J. B.; Ortiz, J. V.; Cui, Q.; Baboul, A. G.; Clifford, S.; Cioslowski, J.; Stefanov, B. B.; Lui, G.; Laishenko, A.; Piskorz, R.; Komaromi, I.; Martin, R. L.; Fox, D. J.; Keith, T.; Al-Laham, M. A.; Peng, C. Y.; Nanayakkara, A.; Challacombe, M.; Gill, P. M. W.; Johnson, B.; Chen, W.; Wong, M. W.; Gonzalez, C.; Pople, J. A. Gaussin 03, Gaussian, Inc.: Pittsburgh, PA, 2003.

2. $\quad$ Perdew, J. P., Phys. Rev. B 1986, 33, 8822-8824.

3. Becke, A. D., Phys. Rev. A 1988, 38, 3098-3100.

4. $\quad$ Schaefer, A.; Horn, H.; Ahlrichs, R., J. Chem. Phys. 1992, 97, 2571-2577.

5. Schaefer, A.; Huber, C.; Ahlrichs, R., J. Chem. Phys. 1994, 100, 5829-5835.

6. Neese, F. ORCA, 2.2; Max-Planck Institut feur Bioanorganische Chemie: Meulheim/Ruhr, Germany, 2004.

7. Allouche, A.; Pourcin, J., Spectrochim. Acta 1993, 49, 571.

8. $\quad$ Praneeth, V. K. K.; Näther, C.; Peters, G.; Lehnert, N., Inorg. Chem. 2006, 45, 27952811. 
Table S1. Geometric and vibrational parameters of selected $(\mathrm{P}) \mathrm{Fe}(\mathrm{NO})(\mathrm{X})^{0 / 1+}$ complexes. All data is experimental unless otherwise indicated.

\begin{tabular}{|c|c|c|c|c|c|c|c|}
\hline \multirow[b]{2}{*}{ Complex } & \multicolumn{4}{|c|}{ Geometric Parameters $[\AA \AA]\left[{ }^{\circ}\right]$} & \multirow[b]{2}{*}{$\Delta \mathrm{Fe}-\mathrm{Np}$} & \multicolumn{2}{|c|}{ Frequencies $\left[\mathrm{cm}^{-1}\right]$} \\
\hline & $\Delta \mathrm{Fe}-\mathrm{NO}$ & $\Delta \mathrm{N}-\mathrm{O}$ & $<\mathrm{Fe}-\mathrm{X}-\mathrm{O}$ & $\Delta \mathrm{Fe}-\mathrm{X}$ & & $v(\mathrm{~N}-\mathrm{O})$ & $v(\mathrm{Fe}-\mathrm{NO})$ \\
\hline$\overline{c a l c . ~(\mathrm{P}) \mathrm{Fe}(\mathrm{NO})(\mathrm{Melm})^{1+}}$ & 1.644 & 1.147 & 180 & 2.018 & 2.022 & 1933 & 639 \\
\hline$(\mathrm{OEP}) \mathrm{Fe}(\mathrm{NO})(\mathrm{Melm})\left(\mathrm{ClO}_{4}\right)$ & 1.646 & 1.135 & 177 & 1.988 & 2.003 & 1921 & - \\
\hline (TPP)Fe(NO)(Melm) $\left(\mathrm{BF}_{4}\right)$ & - & - & - & - & - & 1896 & 580 \\
\hline calc. $(\mathrm{P}) \mathrm{Fe}(\mathrm{NO})\left(\mathrm{OC}(=\mathrm{O}) \mathrm{CF}_{3}\right)$ & 1.637 & 1.155 & 175.8 & 1.936 & 2.025 & 1898 & 649 \\
\hline calc. $(\mathrm{P}) \mathrm{Fe}(\mathrm{NO})\left(\mathrm{OC}(=\mathrm{O}) \mathrm{CH}_{3}\right)$ & 1.656 & 1.158 & 170.6 & 1.905 & 2.027 & 1871 & 644 \\
\hline$(\mathrm{TPP}) \mathrm{Fe}(\mathrm{NO})\left(\mathrm{OC}(=\mathrm{O}) \mathrm{CF}_{3}\right)$ & 1.618 & 1.151 & 175.8 & 1.899 & 2.011 & 1907 & - \\
\hline calc. $(\mathrm{P}) \mathrm{Fe}(\mathrm{NO})\left(\mathrm{NO}_{2}\right)$ & 1.676 & 1.158 & 165.4 & 2.067 & 2.025 & 1854 & 596 \\
\hline$(\mathrm{TPP}) \mathrm{Fe}(\mathrm{NO})\left(\mathrm{NO}_{2}\right)$ & 1.671 & 1.144 & 169 & 1.998 & 1.996 & 1874 & - \\
\hline calc. (P)Fe(NO)(SPh) & 1.685 & 1.162 & 164.4 & 2.343 & 2.027 & 1829 & 584 \\
\hline$(\mathrm{OEP}) \mathrm{Fe}(\mathrm{NO})\left(\mathrm{SR}-\mathrm{H}_{2}\right)$ & 1.671 & 1.187 & 160 & 2.356 & 2.01 & 1850 & 549 \\
\hline
\end{tabular}

Table S2. Charge contributions of important molecular orbital of $(\mathrm{P}) \mathrm{Fe}(\mathrm{NO})\left(\mathrm{OC}(=\mathrm{O}) \mathrm{CF}_{3}\right)$ calculated with BP86/TZVP.

\begin{tabular}{|c|c|c|c|c|c|c|c|c|}
\hline \multirow[b]{2}{*}{ MO \# } & \multirow[b]{2}{*}{ label } & \multirow[b]{2}{*}{ energy } & \multirow{2}{*}{$\begin{array}{c}\text { Fe } \\
d \\
\end{array}$} & \multicolumn{2}{|c|}{$\mathbf{N}$} & \multicolumn{2}{|c|}{0} & \multirow{2}{*}{$\begin{array}{c}\mathrm{OC}(=0) \mathrm{CF}_{3}{ }^{-} \\
\mathrm{s}+\mathrm{p}\end{array}$} \\
\hline & & & & $\mathbf{s}$ & $\mathbf{p}$ & $\mathbf{s}$ & $\mathbf{p}$ & \\
\hline$<129>$ & $\pi^{*}{ }_{y} d_{y z}$ & -0.15192 & 28.3 & 0 & 40.8 & 0 & 24.3 & 0.8 \\
\hline$<128>$ & $\pi^{*}{ }_{x} d_{x z}(L U M O)$ & -0.15303 & 31.8 & 0.5 & 36.1 & 0.1 & 21.9 & 3.0 \\
\hline$<127>$ & $\mathrm{A}_{2 \mathrm{u}}(\mathrm{HOMO})$ & -0.19883 & 0.7 & 0.2 & 0.1 & 0 & 0 & 1.5 \\
\hline$<125>$ & $\mathrm{OC}(=\mathrm{O}) \mathrm{CF}_{3}^{-}(\sigma)+\mathrm{d}_{\mathrm{z}^{2}-\sigma^{*}}$ & -0.2196 & 3.1 & 0.3 & 0.1 & 0 & 0.1 & 4.0 \\
\hline$<115>$ & $d_{y z} \pi^{*}{ }_{y}$ & -0.2998 & 46.9 & 0 & 3.6 & 0 & 10.6 & 3.0 \\
\hline$<114>$ & $d_{x z} \pi_{x}^{*}$ & -0.30115 & 48.2 & 0 & 4.0 & 0 & 12.2 & 6.0 \\
\hline$<71>$ & $\sigma_{\mathrm{nb} \_} \mathrm{d}_{\mathrm{z} 2}$ & -0.53473 & 4.8 & 2.6 & 18.2 & 11.4 & 32.3 & 1.9 \\
\hline
\end{tabular}


Table S3. BP86/TZVP calculated force constants and stretching frequencies of $(\mathrm{P}) \mathrm{Fe}(\mathrm{NO})(\mathrm{X})^{0 / 1+}$ complexes.

\begin{tabular}{|c|c|c|c|c|c|}
\hline \multirow[b]{2}{*}{ Complex } & \multicolumn{3}{|c|}{ Calculated Force Constants (mdyn/Å) } & \multicolumn{2}{|c|}{ Calculated Frequencies $\left(\mathrm{cm}^{-1}\right)$} \\
\hline & $\mathrm{N}-\mathrm{O}$ & Fe-NO & $\mathrm{Fe}-\mathrm{X}^{a}$ & $v(\mathrm{~N}-\mathrm{O})$ & $v(\mathrm{Fe}-\mathrm{NO})$ \\
\hline$(\mathrm{P}) \mathrm{Fe}(\mathrm{NO})(\mathrm{Melm})^{1+}$ & 15.62 & 4.82 & 1.48 & 1933 & 639 \\
\hline$(\mathrm{P}) \mathrm{Fe}(\mathrm{NO})\left(\mathrm{OC}(=\mathrm{O}) \mathrm{CF}_{3}\right)$ & 14.87 & 5.14 & 1.62 & 1898 & 649 \\
\hline$(\mathrm{P}) \mathrm{Fe}(\mathrm{NO})\left(\mathrm{OC}(=\mathrm{O}) \mathrm{CH}_{3}\right)$ & 14.54 & 4.67 & 1.90 & 1871 & 644 \\
\hline$(\mathrm{P}) \mathrm{Fe}(\mathrm{NO})\left(\mathrm{NO}_{2}\right)$ & 14.43 & 4.24 & 1.29 & 1854 & 596 \\
\hline$(\mathrm{P}) \mathrm{Fe}(\mathrm{NO})(\mathrm{SPh})$ & 14.03 & 3.99 & 1.16 & 1829 & 584 \\
\hline
\end{tabular}

Table S4. Charge contributions of important molecular orbital of $(\mathrm{P}) \mathrm{Fe}(\mathrm{NO})(\mathrm{X})^{0 / 1+}$ calculated with BP86/TZVP.

\begin{tabular}{|c|c|c|c|c|c|c|c|c|c|c|c|c|c|c|c|}
\hline \multirow[b]{3}{*}{ Complex } & \multirow[b]{3}{*}{ MO } & \multicolumn{6}{|c|}{$\pi$-backbond } & \multirow[b]{3}{*}{ MO } & \multicolumn{6}{|c|}{$d z^{2} / d x z \_\sigma^{*}$} & \multirow[b]{3}{*}{ ref } \\
\hline & & \multirow{2}{*}{$\begin{array}{c}\mathrm{Fe} \\
\mathrm{d}\end{array}$} & \multicolumn{2}{|c|}{$\mathbf{N}$} & \multicolumn{2}{|c|}{0} & \multirow{2}{*}{$\begin{array}{c}X \\
s+p\end{array}$} & & \multirow{2}{*}{$\begin{array}{c}\mathrm{Fe} \\
\mathrm{d}\end{array}$} & \multicolumn{2}{|c|}{$\mathbf{N}$} & \multicolumn{2}{|c|}{0} & \multirow{2}{*}{$\begin{array}{c}X \\
s+p\end{array}$} & \\
\hline & & & $\mathbf{s}$ & $\mathbf{p}$ & $\mathbf{s}$ & $\mathbf{p}$ & & & & $\mathbf{s}$ & p & $\mathbf{s}$ & $\mathbf{p}$ & & \\
\hline$(\mathrm{P}) \mathrm{Fe}(\mathrm{NO})(\mathrm{Melm})^{1+}$ & $\begin{array}{l}<124>\pi^{*} \text { dyz } \\
<123>\pi^{*} d x z\end{array}$ & $\begin{array}{l}27 \\
27\end{array}$ & $\begin{array}{l}0 \\
0\end{array}$ & $\begin{array}{l}42 \\
42\end{array}$ & $\begin{array}{l}0 \\
0\end{array}$ & $\begin{array}{l}26 \\
26\end{array}$ & $\begin{array}{l}0 \\
0\end{array}$ & $<122>$ & 1 & 0.2 & 0.1 & 0 & 0 & 2 & 1 \\
\hline \multirow[t]{2}{*}{$(\mathrm{P}) \mathrm{Fe}(\mathrm{NO})\left(\mathrm{OC}(=\mathrm{O}) \mathrm{CF}_{3}\right)$} & $<129>\pi^{*}{ }^{*}$ dyz & 28.3 & 0 & 40.8 & 0 & 24.3 & 0.8 & $<125>$ & 3.1 & 0.3 & 0.1 & 0 & 0.1 & 4 & tw \\
\hline & $<128>\pi^{*}{ }^{*} d x z$ & 31.8 & 0.5 & 36.1 & 0.1 & 21.9 & 3 & & & & & & & & \\
\hline$(\mathrm{P}) \mathrm{Fe}(\mathrm{NO})\left(\mathrm{NO}_{2}\right)$ & $<113>\pi^{*}{ }^{-} d x z$ & 27.5 & 0 & 41.5 & 0 & 24.6 & 0 & $<108>+<109>$ & 14.6 & 1.4 & 1 & 0.1 & 0.9 & 8 & tw \\
\hline \multirow[t]{2}{*}{ (P)Fe(NO)(SPh) } & $<130>\pi^{*}{ }^{*} d z^{2} / d x z$ & 25.4 & 0 & 42.2 & 0 & 25 & 0.1 & $<124>+<127>$ & 14.6 & 1.5 & 1.3 & 0 & 1.1 & 26.6 & 2 \\
\hline & $<129>\pi^{*} \_\bar{d} y z \_p z(S)$ & 31.8 & 0.8 & 28.3 & 0.2 & 17.4 & 10.8 & & & & & & & & \\
\hline
\end{tabular}

1. Praneeth et al. J. Am. Chem. Soc. 2008, 130, 15288-15303

2. Paulat et al. Inorg. Chem. 2007, 46, 1547-1549 
Part B: X-ray Crystallographic Determination of $(\mathrm{TPP}) \mathrm{Fe}\left(\mathrm{OC}(=\mathbf{O}) \mathrm{CF}_{3}\right)(1)$

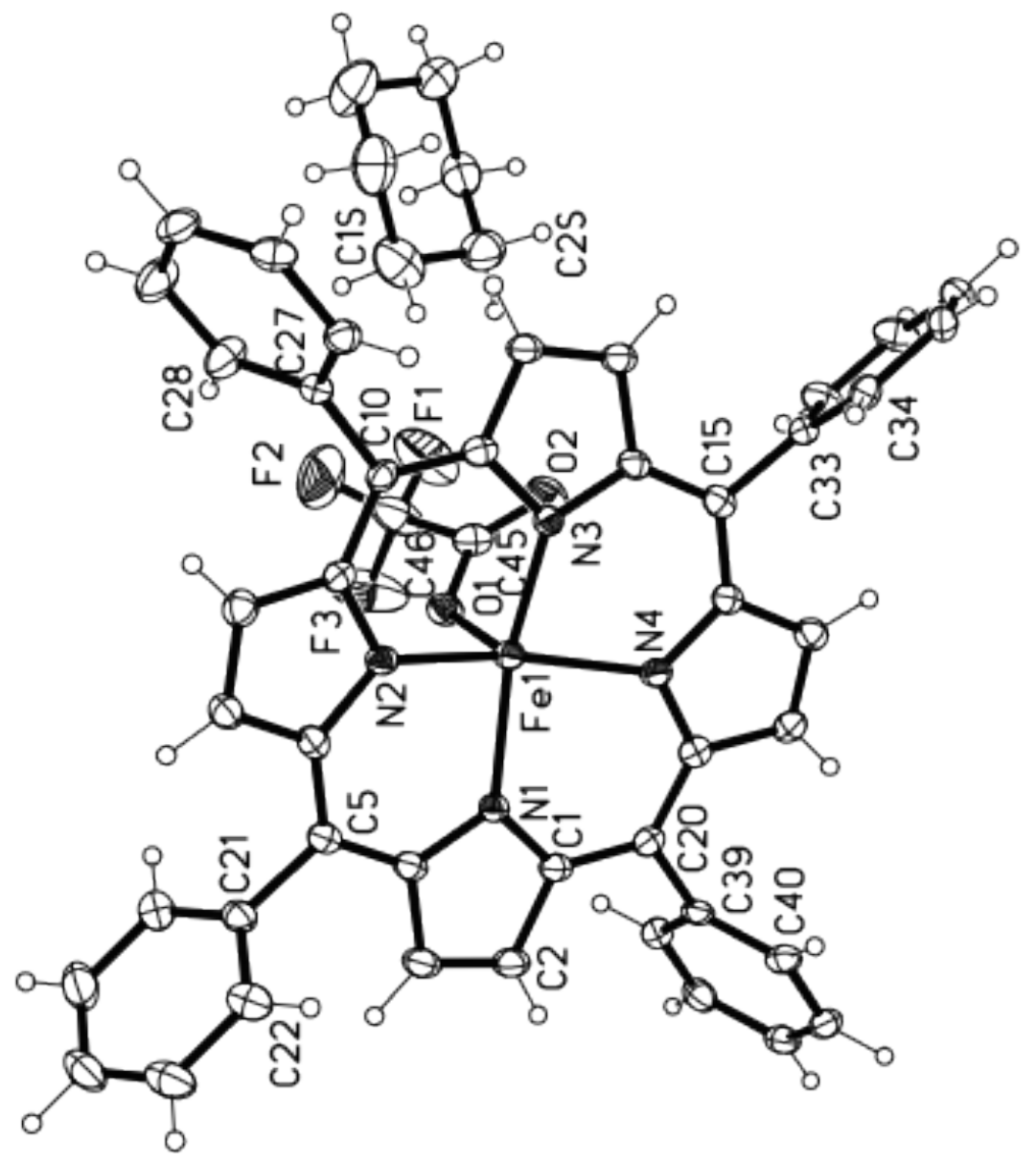

Figure S1. Molecular structure of (TPP) $\mathrm{Fe}\left(\mathrm{OC}(=\mathrm{O}) \mathrm{CF}_{3}\right)(\mathbf{1})$. 


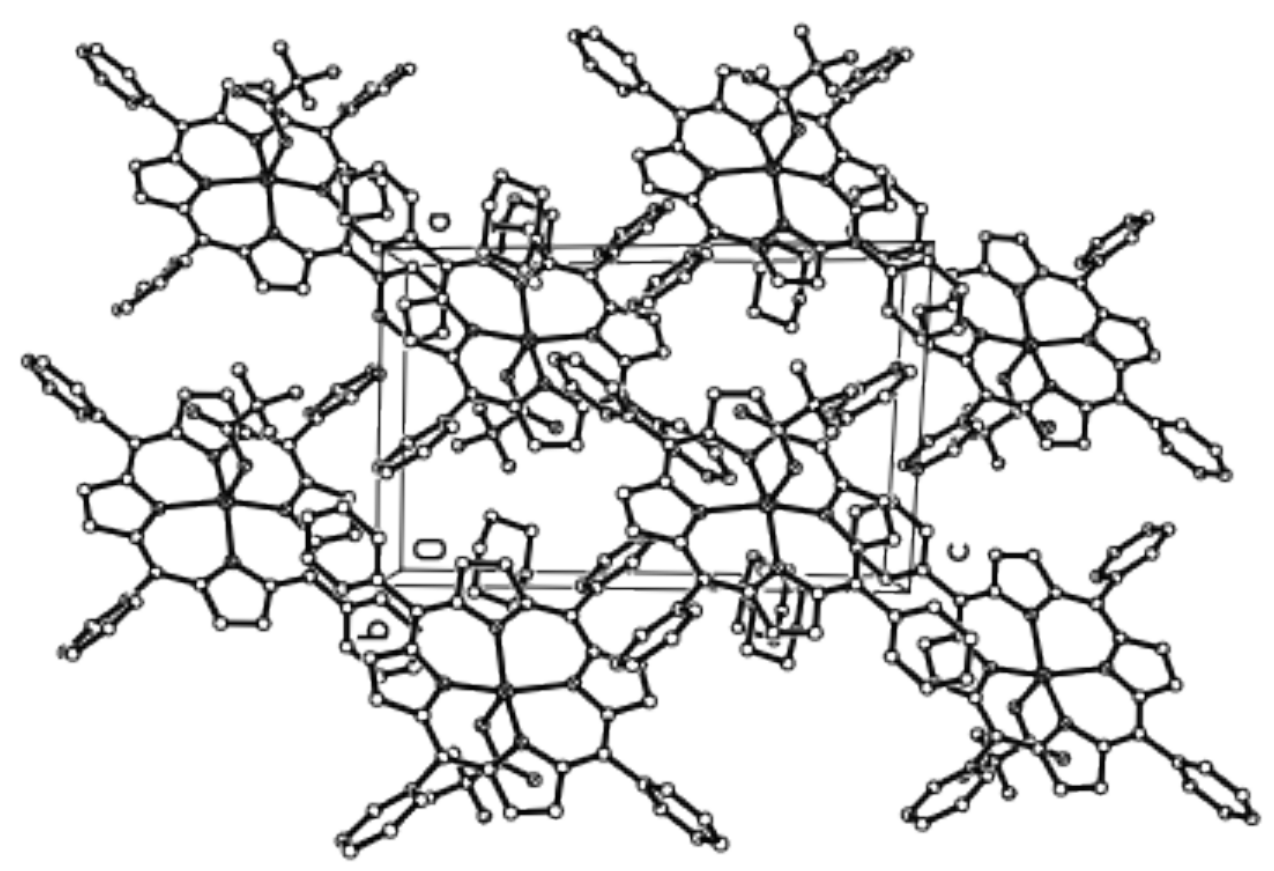

Figure S2. Crystal packing of (TPP $) \mathrm{Fe}\left(\mathrm{OC}(=\mathrm{O}) \mathrm{CF}_{3}\right)(\mathbf{1})$, with displacement ellipsoids drawn at the $50 \%$ probability level. 

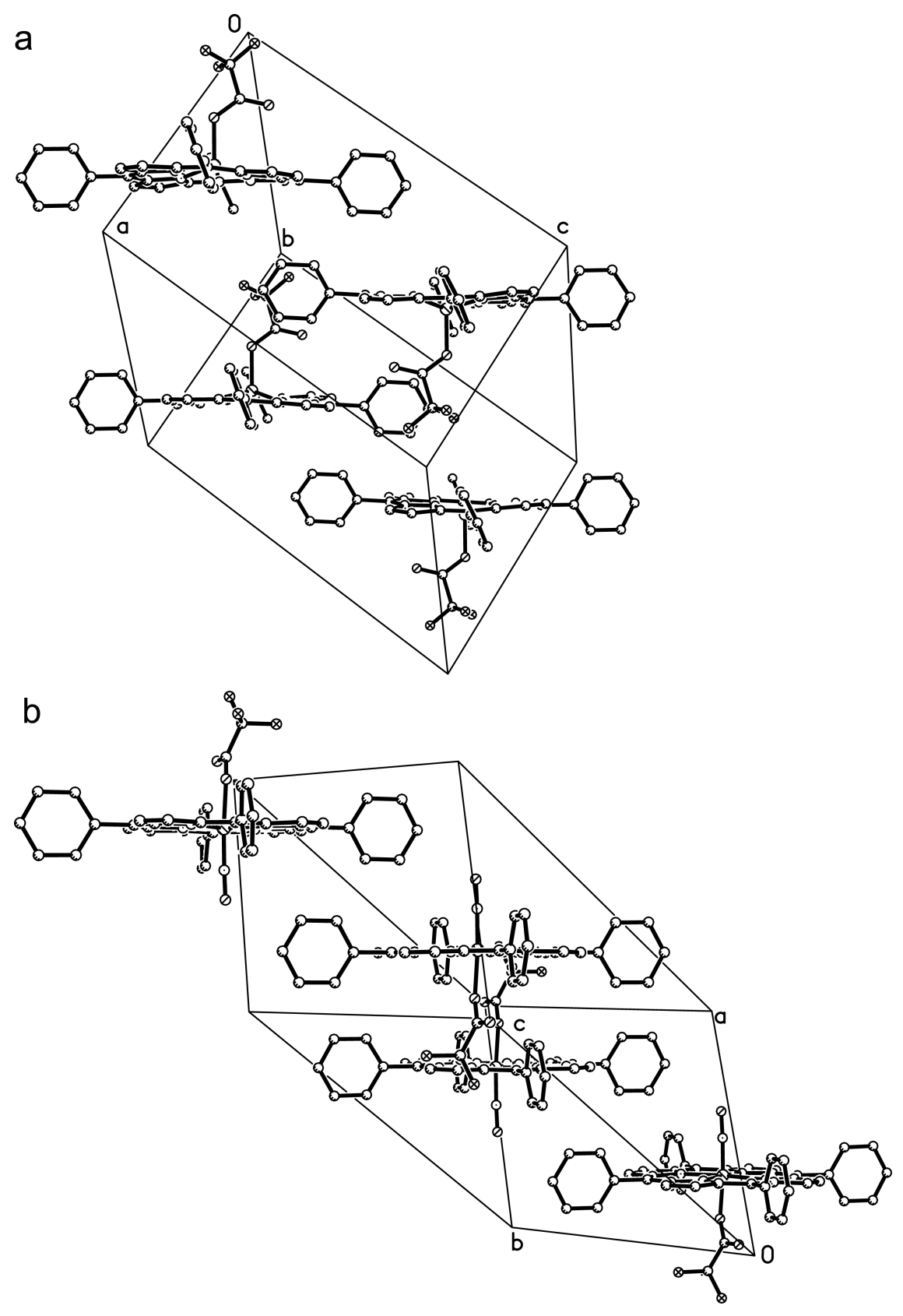

Figure S3. Comparison of the unit cell of $(\mathrm{TPP}) \mathrm{Fe}\left(\mathrm{OC}(=\mathrm{O}) \mathrm{CF}_{3}\right)(\mathbf{1}$; a) with that of (TPP) $\mathrm{Fe}(\mathrm{NO})\left(\mathrm{OC}(=\mathrm{O}) \mathrm{CF}_{3}\right)(2 ; \mathrm{b})$ 


\section{Experimental}

A black prism-shaped crystal of dimensions $0.30 \times 0.30 \times 0.26 \mathrm{~mm}$ was selected for structural analysis. Intensity data for this compound were collected using a diffractometer with a Bruker APEX ccd area detector (1) and graphite-monochromated Mo $\mathrm{K} \alpha$ radiation $(\lambda=0.71073 \AA)$. The sample was cooled to $100(2) \mathrm{K}$. Cell parameters were determined from a non-linear least squares fit of 8966 peaks in the range $2.25<\theta<28.29^{\circ}$. A total of 24156 data were measured in the range $1.86<\theta<28.31^{\circ}$ using $\Phi$ and $\omega$ oscillation frames. The data were corrected for absorption by the Semi-empirical from equivalents method (2) giving minimum and maximum transmission factors of 0.8840 and 0.8983 . The data were merged to form a set of 10322 independent data with $\mathrm{R}$ (int) $=0.0371$ and a coverage of $100.0 \%$.

The Triclinic space group $P \overline{1}$ was determined by systematic absences and statistical tests and verified by subsequent refinement. The structure was solved by direct methods and refined by full-matrix least-squares methods on $F^{2}(3)$. Hydrogen atom positions of hydrogens bonded to carbons were initially determined by geometry and refined by a riding model. Hydrogens bonded to nitrogens and oxygens were located on a difference map, and their positions were refined independently. Non-hydrogen atoms were refined with anisotropic displacement parameters. Hydrogen atom displacement parameters were set to 1.2 (1.5 for methyl) times the isotropic equivalent displacement parameters of the bonded atoms. A total of 563 parameters were refined against 0 restraints and 10322 data to give $\mathrm{wR}\left(\mathrm{F}^{2}\right)=0.1407$ and $\mathrm{S}=0.986$ for weights of $\mathrm{w}=1 /\left[\sigma^{2}\left(\mathrm{~F}^{2}\right)+(? \mathrm{P}) 2+? \mathrm{P}\right]$, where $\mathrm{P}=\left[\mathrm{Fo}^{2}\right.$

$\left.+2 \mathrm{Fc}^{2}\right] / 3$. The final $\mathrm{R}(\mathrm{F})$ was 0.0524 for the 7973 observed, $[\mathrm{F}>4 \sigma(\mathrm{F})]$, data. The largest shift/s.u. was 0.000 in the final refinement cycle. The final difference map had maxima and minima of 0.564 and $-0.513 \mathrm{e} / \AA^{3}$, respectively. The absolute structure was determined by refinement of the Flack parameter (4). The polar axis restraints were taken from Flack and Schwarzenbach (5).

Note: In the difference map before this model, a large $\left(>3 \mathrm{e}-/ \AA^{3}\right)$ peak was located near ( $0.99 \AA$ from the iron, across the TPP ring). In this model, this extra peak is modeled as disordered Fe (i.e., Fel in the Tables). Note that the trifluoroacetate bonded to this alternate Fe could not be located in the subsequent difference map.

\section{References}

(1) (a) Data Collection: SMART Software Reference Manual (2007). Bruker-AXS, 5465 E. Cheryl Parkway, Madison, WI 53711-5373, USA. (b) Data Reduction: SAINT 
Software Reference Manual (2007). B ruker-AXS, 5465 E. Cheryl Parkway, Madison, WI 53711-5373 USA.

(2) G. M. Sheldrick (2001). SADABS. Program for Empirical Absorption Correction of Area Detector Data. University of Göttingen, Germany.

(3) G. M. Sheldrick (2008). Acta Cryst., A64, 112-122.

(4) H. D. Flack (1983). Acta Cryst. A39, 876-881.

(5) H. D. Flack and D. Schwarzenbach (1988). Acta Cryst. A44, 499-506.

(6) P. van der Sluis and A. L. Spek (1990). Acta Cryst. A46, 194-201. 
Table S5. Crystal data and structure refinement for $\mathbf{1} \bullet\left(\mathrm{C}_{6} \mathrm{H}_{12}\right)$

Empirical formula

Formula weight

Crystal system

Space group

Unit cell dimensions

Volume

Z, Z'

Density (calculated)

Wavelength

Temperature

$\mathrm{F}(000)$

Absorption coefficient

Absorption correction

Max. and min. transmission

Theta range for data collection

Reflections collected

Independent reflections

Data / restraints / parameters

wR(F2 all data)

$\mathrm{R}$ (F obsd data)

Goodness-of-fit on F2

Observed data [I $>2 \mathrm{~s}(\mathrm{I})]$

Largest and mean shift / s.u.

Largest diff. peak and hole
$\mathrm{C}_{52} \mathrm{H}_{40} \mathrm{~F}_{3} \mathrm{Fe}_{1} \mathrm{~N}_{4} \mathrm{O}_{2}$

865.73

Triclinic

$P-1$

$a=11.2285(9) \AA \quad \alpha=72.885(2)^{\circ}$

$b=12.0522(9) \AA \quad \beta=82.233(2)^{\circ}$

$c=17.1643(13) \AA \quad Y=70.680(2)^{\circ}$

2093.1(3) $\AA^{3}$

2

$1.374 \mathrm{Mg} / \mathrm{m} 3$

$0.71073 \AA$

$100(2) \mathrm{K}$

898

$0.421 \mathrm{~mm}^{-1}$

Semi-empirical from equivalents

0.8983 and 0.8840

1.86 to $28.31^{\circ}$

24156

$10322[R$ (int) $=0.0371]$

10322 / 0 / 563

$w R 2=0.1407$

$\mathrm{R} 1=0.0524$

0.986

7973

0.000 and 0.000

0.564 and $-0.513 \mathrm{e} / \AA^{3}$

$\mathrm{wR} 2=\left\{\Sigma\left[\mathrm{w}\left(\mathrm{F}_{\mathrm{o}}^{2}-\mathrm{F}_{\mathrm{c}}^{2}\right)^{2}\right] / \Sigma\left[\mathrm{w}\left(\mathrm{F}_{\mathrm{o}}^{2}\right)^{2}\right]\right\}^{1 / 2}$

$\mathrm{R} 1=\Sigma|| \mathrm{F}_{\mathrm{o}}|-| \mathrm{F}_{\mathrm{c}}|| / \Sigma\left|\mathrm{F}_{\mathrm{o}}\right|$ 
Table S6. Atomic coordinates and equivalent isotropic displacement parameters for 1. $U(e q)$ is defined as one third of the trace of the orthogonalized $U_{i j}$ tensor.

\begin{tabular}{|c|c|c|c|c|}
\hline & $x$ & $y$ & $\mathrm{U}(\mathrm{eq})$ & \\
\hline $\mathrm{Fe}(1)$ & $0.26209(3)$ & $0.30443(3)$ & $0.726616(19)$ & $0.01530(10)$ \\
\hline $\mathrm{Fe}\left(1^{\prime}\right)$ & $0.1973(6)$ & $0.2668(5)$ & $0.7193(4)$ & $0.01530(10)$ \\
\hline$F(1)$ & $0.64297(19)$ & $0.4450(2)$ & $0.76183(14)$ & $0.0734(7)$ \\
\hline$F(2)$ & $0.5785(2)$ & $0.3210(2)$ & $0.85968(13)$ & $0.0791(7)$ \\
\hline$F(3)$ & $0.46639(19)$ & $0.5069(2)$ & $0.82427(17)$ & $0.0887(9)$ \\
\hline $\mathrm{O}(1)$ & $0.36672(15)$ & $0.37140(15)$ & $0.76814(10)$ & $0.0253(4)$ \\
\hline $\mathrm{O}(2)$ & $0.52313(17)$ & $0.37749(16)$ & $0.67049(11)$ & $0.0332(4)$ \\
\hline $\mathrm{N}(1)$ & $0.08486(17)$ & $0.41629(16)$ & $0.74828(11)$ & $0.0183(4)$ \\
\hline $\mathrm{N}(2)$ & $0.23232(17)$ & $0.17847(16)$ & $0.83318(11)$ & $0.0185(4)$ \\
\hline $\mathrm{N}(3)$ & $0.39282(17)$ & $0.16133(16)$ & $0.69140(11)$ & $0.0180(4)$ \\
\hline $\mathrm{N}(4)$ & $0.24192(17)$ & $0.39711(16)$ & $0.60553(11)$ & $0.0187(4)$ \\
\hline$C(1)$ & $0.0230(2)$ & $0.52865(19)$ & $0.69817(14)$ & $0.0202(4)$ \\
\hline$C(2)$ & $-0.0868(2)$ & $0.5897(2)$ & $0.74096(15)$ & $0.0257(5)$ \\
\hline$C(3)$ & $-0.0913(2)$ & $0.5150(2)$ & $0.81645(15)$ & $0.0272(5)$ \\
\hline$C(4)$ & $0.0147(2)$ & $0.4063(2)$ & $0.82117(14)$ & $0.0207(5)$ \\
\hline$C(5)$ & $0.0381(2)$ & $0.3052(2)$ & $0.88918(14)$ & $0.0201(5)$ \\
\hline$C(6)$ & $0.1375(2)$ & $0.1978(2)$ & $0.89275(13)$ & $0.0196(4)$ \\
\hline$C(7)$ & $0.1578(2)$ & $0.0912(2)$ & $0.96069(14)$ & $0.0229(5)$ \\
\hline $\mathrm{C}(8)$ & $0.2656(2)$ & $0.0080(2)$ & $0.94329(14)$ & $0.0236(5)$ \\
\hline$C(9)$ & $0.3129(2)$ & $0.06231(19)$ & $0.86421(13)$ & $0.0191(4)$ \\
\hline$C(10)$ & $0.4236(2)$ & $0.00525(19)$ & $0.82462(13)$ & $0.0190(4)$ \\
\hline$C(11)$ & $0.4607(2)$ & $0.05363(19)$ & $0.74393(14)$ & $0.0193(4)$ \\
\hline$C(12)$ & $0.5745(2)$ & $-0.0031(2)$ & $0.70220(14)$ & $0.0220(5)$ \\
\hline$C(13)$ & $0.5743(2)$ & $0.0676(2)$ & $0.62538(14)$ & $0.0212(5)$ \\
\hline$C(14)$ & $0.4616(2)$ & $0.17028(19)$ & $0.61746(13)$ & $0.0186(4)$ \\
\hline$C(15)$ & $0.4295(2)$ & $0.26527(19)$ & $0.54664(14)$ & $0.0194(4)$ \\
\hline$C(16)$ & $0.3261(2)$ & 0.37087(19) & $0.54161(13)$ & $0.0186(4)$ \\
\hline$C(17)$ & $0.2946(2)$ & $0.4698(2)$ & $0.46913(14)$ & $0.0222(5)$ \\
\hline$C(18)$ & $0.1924(2)$ & $0.5566(2)$ & $0.48917(14)$ & $0.0225(5)$ \\
\hline$C(19)$ & $0.1588(2)$ & $0.51157(19)$ & $0.57342(13)$ & $0.0194(4)$ \\
\hline$C(20)$ & $0.0578(2)$ & $0.57556(19)$ & $0.61663(14)$ & $0.0194(4)$ \\
\hline$C(21)$ & $-0.0518(2)$ & $0.3136(2)$ & $0.96179(14)$ & $0.0228(5)$ \\
\hline $\mathrm{C}(22)$ & $-0.1691(2)$ & $0.2981(2)$ & $0.96111(17)$ & $0.0311(6)$ \\
\hline $\mathrm{C}(23)$ & $-0.2558(3)$ & $0.3105(2)$ & $1.02647(18)$ & $0.0360(6)$ \\
\hline $\mathrm{C}(24)$ & $-0.2256(3)$ & $0.3395(2)$ & $1.09174(17)$ & $0.0388(7)$ \\
\hline$C(25)$ & $-0.1095(3)$ & $0.3564(3)$ & $1.09240(17)$ & $0.0400(7)$ \\
\hline$C(26)$ & $-0.0227(3)$ & $0.3431(2)$ & $1.02706(15)$ & $0.0316(6)$ \\
\hline $\mathrm{C}(27)$ & $0.5059(2)$ & $-0.11513(19)$ & $0.87179(14)$ & $0.0204(5)$ \\
\hline
\end{tabular}




\begin{tabular}{lllll}
$\mathrm{C}(28)$ & $0.5674(3)$ & $-0.1210(2)$ & $0.93822(16)$ & $0.0307(6)$ \\
$\mathrm{C}(29)$ & $0.6389(3)$ & $-0.2322(2)$ & $0.98601(17)$ & $0.0351(6)$ \\
$\mathrm{C}(30)$ & $0.6511(2)$ & $-0.3393(2)$ & $0.96630(15)$ & $0.0282(5)$ \\
$\mathrm{C}(31)$ & $0.5922(2)$ & $-0.3339(2)$ & $0.89910(15)$ & $0.0253(5)$ \\
$\mathrm{C}(32)$ & $0.5195(2)$ & $-0.2230(2)$ & $0.85206(14)$ & $0.0221(5)$ \\
$\mathrm{C}(33)$ & $0.5128(2)$ & $0.25403(19)$ & $0.47176(13)$ & $0.0189(4)$ \\
$\mathrm{C}(34)$ & $0.5166(2)$ & $0.1662(2)$ & $0.43250(14)$ & $0.0211(5)$ \\
$\mathrm{C}(35)$ & $0.5966(2)$ & $0.1533(2)$ & $0.36469(13)$ & $0.0233(5)$ \\
$\mathrm{C}(36)$ & $0.6737(2)$ & $0.2268(2)$ & $0.33462(14)$ & $0.0255(5)$ \\
$\mathrm{C}(37)$ & $0.6693(2)$ & $0.3152(2)$ & $0.37272(16)$ & $0.0294(6)$ \\
$\mathrm{C}(38)$ & $0.5900(2)$ & $0.3280(2)$ & $0.44107(15)$ & $0.0268(5)$ \\
$\mathrm{C}(39)$ & $-0.0180(2)$ & $0.7023(2)$ & $0.57448(14)$ & $0.0206(5)$ \\
$\mathrm{C}(40)$ & $-0.0926(2)$ & $0.7235(2)$ & $0.51008(14)$ & $0.0229(5)$ \\
$\mathrm{C}(41)$ & $-0.1637(2)$ & $0.8421(2)$ & $0.47189(15)$ & $0.0257(5)$ \\
$\mathrm{C}(42)$ & $-0.1595(2)$ & $0.9391(2)$ & $0.49772(15)$ & $0.0271(5)$ \\
$\mathrm{C}(43)$ & $-0.0863(2)$ & $0.9186(2)$ & $0.56255(15)$ & $0.0273(5)$ \\
$\mathrm{C}(44)$ & $-0.0162(2)$ & $0.8006(2)$ & $0.60069(14)$ & $0.0242(5)$ \\
$\mathrm{C}(45)$ & $0.4737(2)$ & $0.3855(2)$ & $0.73635(16)$ & $0.0259(5)$ \\
$\mathrm{C}(46)$ & $0.5410(3)$ & $0.4162(3)$ & $0.7949(2)$ & $0.0449(8)$ \\
$\mathrm{C}(1 \mathrm{~S})$ & $0.7910(3)$ & $0.0978(3)$ & $0.7835(2)$ & $0.0490(8)$ \\
$\mathrm{C}(2 \mathrm{~S})$ & $0.8362(3)$ & $0.1798(3)$ & $0.70737(19)$ & $0.0425(7)$ \\
$\mathrm{C}(3 \mathrm{~S})$ & $0.9688(3)$ & $0.1793(3)$ & $0.71612(18)$ & $0.0391(7)$ \\
$\mathrm{C}(4 \mathrm{~S})$ & $1.0603(3)$ & $0.0508(3)$ & $0.73388(18)$ & $0.0419(7)$ \\
$\mathrm{C}(5 \mathrm{~S})$ & $1.0142(3)$ & $-0.0320(3)$ & $0.8087(2)$ & $0.0581(9)$ \\
$\mathrm{C}(6 \mathrm{~S})$ & $0.8817(3)$ & $-0.0316(3)$ & $0.8002(2)$ & $0.0502(8)$ \\
& & & & \\
\hline
\end{tabular}


Table S7. Bond lengths $[\AA]]$ and angles $\left[^{\circ}\right]$ for 1 .

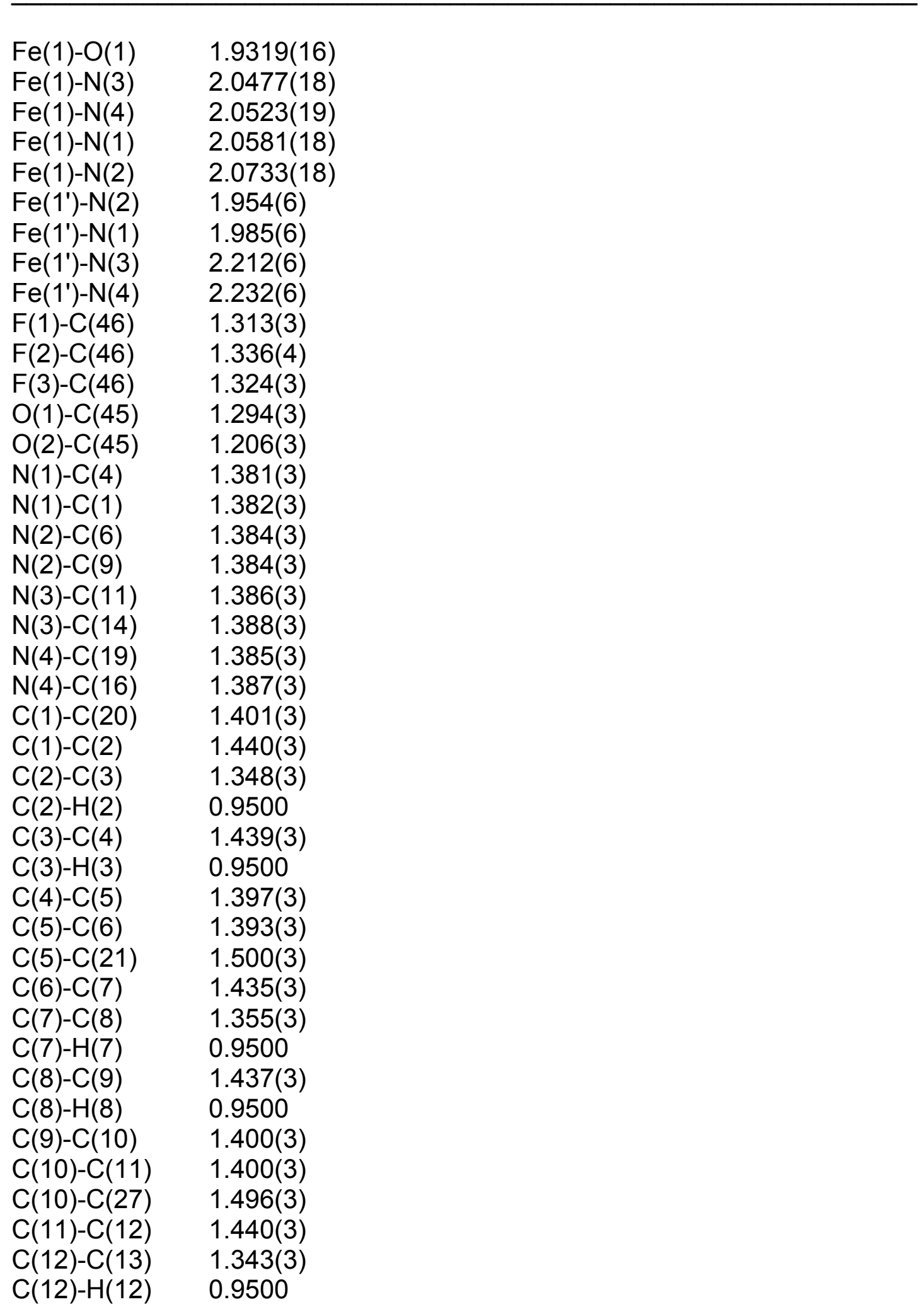




$\begin{array}{ll}\mathrm{C}(13)-\mathrm{C}(14) & 1.436(3) \\ \mathrm{C}(13)-\mathrm{H}(13) & 0.9500 \\ \mathrm{C}(14)-\mathrm{C}(15) & 1.394(3) \\ \mathrm{C}(15)-\mathrm{C}(16) & 1.400(3) \\ \mathrm{C}(15)-\mathrm{C}(33) & 1.496(3) \\ \mathrm{C}(16)-\mathrm{C}(17) & 1.436(3) \\ \mathrm{C}(17)-\mathrm{C}(18) & 1.357(3) \\ \mathrm{C}(17)-\mathrm{H}(17) & 0.9500 \\ \mathrm{C}(18)-\mathrm{C}(19) & 1.433(3) \\ \mathrm{C}(18)-\mathrm{H}(18) & 0.9500 \\ \mathrm{C}(19)-\mathrm{C}(20) & 1.401(3) \\ \mathrm{C}(20)-\mathrm{C}(39) & 1.495(3) \\ \mathrm{C}(21)-\mathrm{C}(26) & 1.378(4) \\ \mathrm{C}(21)-\mathrm{C}(22) & 1.391(3) \\ \mathrm{C}(22)-\mathrm{C}(23) & 1.392(3) \\ \mathrm{C}(22)-\mathrm{H}(22) & 0.9500 \\ \mathrm{C}(23)-\mathrm{C}(24) & 1.380(4) \\ \mathrm{C}(23)-\mathrm{H}(23) & 0.9500 \\ \mathrm{C}(24)-\mathrm{C}(25) & 1.386(4) \\ \mathrm{C}(24)-\mathrm{H}(24) & 0.9500 \\ \mathrm{C}(25)-\mathrm{C}(26) & 1.393(4) \\ \mathrm{C}(25)-\mathrm{H}(25) & 0.9500 \\ \mathrm{C}(26)-\mathrm{H}(26) & 0.9500 \\ \mathrm{C}(27)-\mathrm{C}(28) & 1.384(3) \\ \mathrm{C}(27)-\mathrm{C}(32) & 1.393(3) \\ \mathrm{C}(28)-\mathrm{C}(29) & 1.390(3) \\ \mathrm{C}(28)-\mathrm{H}(28) & 0.9500 \\ \mathrm{C}(29)-\mathrm{C}(30) & 1.388(3) \\ \mathrm{C}(29)-\mathrm{H}(29) & 0.9500 \\ \mathrm{C}(30)-\mathrm{C}(31) & 1.381(3) \\ \mathrm{C}(30)-\mathrm{H}(30) & 0.9500 \\ \mathrm{C}(31)-\mathrm{C}(32) & 1.388(3) \\ \mathrm{C}(31)-\mathrm{H}(31) & 0.9500 \\ \mathrm{C}(32)-\mathrm{H}(32) & 0.9500 \\ \mathrm{C}(33)-\mathrm{C}(38) & 1.392(3) \\ \mathrm{C}(33)-\mathrm{C}(34) & 1.399(3) \\ \mathrm{C}(34)-\mathrm{C}(35) & 1.384(3) \\ \mathrm{C}(34)-\mathrm{H}(34) & 0.9500 \\ \mathrm{C}(35)-\mathrm{C}(36) & 1.385(3) \\ \mathrm{C}(35)-\mathrm{H}(35) & 0.9500 \\ \mathrm{C}(36)-\mathrm{C}(37) & 1.389(3) \\ \mathrm{C}(36)-\mathrm{H}(36) & 0.9500 \\ \mathrm{C}(37)-\mathrm{C}(38) & 1.388(3) \\ \mathrm{C}(37)-\mathrm{H}(37) & 0.9500 \\ \mathrm{C}(38)-\mathrm{H}(38) & 0.9500 \\ & \end{array}$




\begin{tabular}{|c|c|c|}
\hline$C(39)-C(40)$ & $1.390(3)$ & \\
\hline$C(39)-C(44)$ & $1.393(3)$ & \\
\hline$C(40)-C(41)$ & $1.395(3)$ & \\
\hline $\mathrm{C}(40)-\mathrm{H}(40)$ & 0.9500 & \\
\hline$C(41)-C(42)$ & $1.383(4)$ & \\
\hline $\mathrm{C}(41)-\mathrm{H}(41)$ & 0.9500 & \\
\hline$C(42)-C(43)$ & $1.387(4)$ & \\
\hline $\mathrm{C}(42)-\mathrm{H}(42)$ & 0.9500 & \\
\hline$C(43)-C(44)$ & $1.387(3)$ & \\
\hline $\mathrm{C}(43)-\mathrm{H}(43)$ & 0.9500 & \\
\hline $\mathrm{C}(44)-\mathrm{H}(44)$ & 0.9500 & \\
\hline$C(45)-C(46)$ & $1.526(4)$ & \\
\hline$C(1 S)-C(6 S)$ & $1.522(5)$ & \\
\hline$C(1 S)-C(2 S)$ & $1.527(4)$ & \\
\hline $\mathrm{C}(1 \mathrm{~S})-\mathrm{H}(1 \mathrm{~S} 1)$ & 0.9900 & \\
\hline $\mathrm{C}(1 \mathrm{~S})-\mathrm{H}(1 \mathrm{~S} 2)$ & 0.9900 & \\
\hline$C(2 S)-C(3 S)$ & $1.515(4)$ & \\
\hline $\mathrm{C}(2 \mathrm{~S})-\mathrm{H}(2 \mathrm{~S} 1)$ & 0.9900 & \\
\hline $\mathrm{C}(2 \mathrm{~S})-\mathrm{H}(2 \mathrm{~S} 2)$ & 0.9900 & \\
\hline $\mathrm{C}(3 S)-\mathrm{C}(4 S)$ & $1.517(4)$ & \\
\hline $\mathrm{C}(3 \mathrm{~S})-\mathrm{H}(3 \mathrm{~S} 1)$ & 0.9900 & \\
\hline $\mathrm{C}(3 \mathrm{~S})-\mathrm{H}(3 \mathrm{~S} 2)$ & 0.9900 & \\
\hline$C(4 S)-C(5 S)$ & $1.523(4)$ & \\
\hline $\mathrm{C}(4 \mathrm{~S})-\mathrm{H}(4 \mathrm{~S} 1)$ & 0.9900 & \\
\hline $\mathrm{C}(4 \mathrm{~S})-\mathrm{H}(4 \mathrm{~S} 2)$ & 0.9900 & \\
\hline$C(5 S)-C(6 S)$ & $1.512(5)$ & \\
\hline $\mathrm{C}(5 \mathrm{~S})-\mathrm{H}(5 \mathrm{~S} 1)$ & 0.9900 & \\
\hline $\mathrm{C}(5 \mathrm{~S})-\mathrm{H}(5 \mathrm{~S} 2)$ & 0.9900 & \\
\hline $\mathrm{C}(6 \mathrm{~S})-\mathrm{H}(6 \mathrm{SA})$ & 0.9900 & \\
\hline $\mathrm{C}(6 \mathrm{~S})-\mathrm{H}(6 \mathrm{SB})$ & 0.9900 & \\
\hline $\mathrm{O}(1)-\mathrm{Fe}(1)-\mathrm{N}(3)$ & & $102.21(7)$ \\
\hline $\mathrm{O}(1)-\mathrm{Fe}(1)-\mathrm{N}(4)$ & & $105.86(7)$ \\
\hline $\mathrm{N}(3)-\mathrm{Fe}(1)-\mathrm{N}(4)$ & & $88.21(7)$ \\
\hline $\mathrm{O}(1)-\mathrm{Fe}(1)-\mathrm{N}(1)$ & & $100.93(7)$ \\
\hline $\mathrm{N}(3)-\mathrm{Fe}(1)-\mathrm{N}(1)$ & & $156.78(7)$ \\
\hline $\mathrm{N}(4)-\mathrm{Fe}(1)-\mathrm{N}(1)$ & & $87.39(7)$ \\
\hline $\mathrm{O}(1)-\mathrm{Fe}(1)-\mathrm{N}(2)$ & & $98.90(7)$ \\
\hline $\mathrm{N}(3)-\mathrm{Fe}(1)-\mathrm{N}(2)$ & & $87.20(7)$ \\
\hline $\mathrm{N}(4)-\mathrm{Fe}(1)-\mathrm{N}(2)$ & & $155.23(7)$ \\
\hline $\mathrm{N}(1)-\mathrm{Fe}(1)-\mathrm{N}(2)$ & & $87.31(7)$ \\
\hline $\mathrm{N}(2)-\mathrm{Fe}\left(1^{\prime}\right)-\mathrm{N}\left(1^{\prime}\right)$ & & $92.8(3)$ \\
\hline $\mathrm{N}(2)-\mathrm{Fe}\left(1^{\prime}\right)-\mathrm{N}(3)$ & & $85.8(2)$ \\
\hline $\mathrm{N}(1)-\mathrm{Fe}\left(1^{\prime}\right)-\mathrm{N}(3)$ & & $146.7(3)$ \\
\hline $\mathrm{N}(2)-\mathrm{Fe}\left(1^{\prime}\right)-\mathrm{N}(4)$ & & 148.6(3) \\
\hline
\end{tabular}




\begin{tabular}{|c|c|}
\hline $\mathrm{N}(1)-\mathrm{Fe}\left(1^{\prime}\right)-\mathrm{N}(4)$ & $84.4(2)$ \\
\hline $\mathrm{N}(3)-\mathrm{Fe}\left(1^{\prime}\right)-\mathrm{N}(4)$ & $79.9(2)$ \\
\hline $\mathrm{C}(45)-\mathrm{O}(1)-\mathrm{Fe}(1)$ & 126.38(16) \\
\hline$C(4)-N(1)-C(1)$ & 105.62(17) \\
\hline $\mathrm{C}(4)-\mathrm{N}(1)-\mathrm{Fe}\left(1^{\prime}\right)$ & $119.7(2)$ \\
\hline $\mathrm{C}(1)-\mathrm{N}(1)-\mathrm{Fe}\left(1^{\prime}\right)$ & $129.7(2)$ \\
\hline $\mathrm{C}(4)-\mathrm{N}(1)-\mathrm{Fe}(1)$ & $126.87(15)$ \\
\hline $\mathrm{C}(1)-\mathrm{N}(1)-\mathrm{Fe}(1)$ & $126.46(14)$ \\
\hline$C(6)-N(2)-C(9)$ & $105.71(17)$ \\
\hline$C(6)-N(2)-F e\left(1^{\prime}\right)$ & $119.3(2)$ \\
\hline $\mathrm{C}(9)-\mathrm{N}(2)-\mathrm{Fe}\left(1^{\prime}\right)$ & $128.5(2)$ \\
\hline $\mathrm{C}(6)-\mathrm{N}(2)-\mathrm{Fe}(1)$ & $127.35(14)$ \\
\hline $\mathrm{C}(9)-\mathrm{N}(2)-\mathrm{Fe}(1)$ & $126.46(14)$ \\
\hline $\mathrm{C}(11)-\mathrm{N}(3)-\mathrm{C}(14)$ & 105.78(17) \\
\hline $\mathrm{C}(11)-\mathrm{N}(3)-\mathrm{Fe}(1)$ & $125.23(14)$ \\
\hline $\mathrm{C}(14)-\mathrm{N}(3)-\mathrm{Fe}(1)$ & $125.13(14)$ \\
\hline $\mathrm{C}(11)-\mathrm{N}(3)-\mathrm{Fe}\left(1^{\prime}\right)$ & $123.5(2)$ \\
\hline $\mathrm{C}(14)-\mathrm{N}(3)-\mathrm{Fe}\left(1^{\prime}\right)$ & $129.4(2)$ \\
\hline$C(19)-N(4)-C(16)$ & $105.43(18)$ \\
\hline $\mathrm{C}(19)-\mathrm{N}(4)-\mathrm{Fe}(1)$ & $126.83(14)$ \\
\hline $\mathrm{C}(16)-\mathrm{N}(4)-\mathrm{Fe}(1)$ & $126.34(14)$ \\
\hline $\mathrm{C}(19)-\mathrm{N}(4)-\mathrm{Fe}\left(1^{\prime}\right)$ & $122.9(2)$ \\
\hline $\mathrm{C}(16)-\mathrm{N}(4)-\mathrm{Fe}\left(1^{\prime}\right)$ & $128.1(2)$ \\
\hline$N(1)-C(1)-C(20)$ & $125.6(2)$ \\
\hline$N(1)-C(1)-C(2)$ & 109.99(19) \\
\hline$C(20)-C(1)-C(2)$ & $124.3(2)$ \\
\hline$C(3)-C(2)-C(1)$ & $107.1(2)$ \\
\hline $\mathrm{C}(3)-\mathrm{C}(2)-\mathrm{H}(2)$ & 126.4 \\
\hline $\mathrm{C}(1)-\mathrm{C}(2)-\mathrm{H}(2)$ & 126.4 \\
\hline$C(2)-C(3)-C(4)$ & $107.3(2)$ \\
\hline $\mathrm{C}(2)-\mathrm{C}(3)-\mathrm{H}(3)$ & 126.3 \\
\hline $\mathrm{C}(4)-\mathrm{C}(3)-\mathrm{H}(3)$ & 126.3 \\
\hline$N(1)-C(4)-C(5)$ & $126.1(2)$ \\
\hline $\mathrm{N}(1)-\mathrm{C}(4)-\mathrm{C}(3)$ & 109.92(19) \\
\hline$C(5)-C(4)-C(3)$ & $123.9(2)$ \\
\hline$C(6)-C(5)-C(4)$ & $124.1(2)$ \\
\hline$C(6)-C(5)-C(21)$ & 118.75(19) \\
\hline$C(4)-C(5)-C(21)$ & 117.12(19) \\
\hline$N(2)-C(6)-C(5)$ & $125.7(2)$ \\
\hline $\mathrm{N}(2)-\mathrm{C}(6)-\mathrm{C}(7)$ & $109.95(19)$ \\
\hline$C(5)-C(6)-C(7)$ & $124.3(2)$ \\
\hline$C(8)-C(7)-C(6)$ & $107.3(2)$ \\
\hline $\mathrm{C}(8)-\mathrm{C}(7)-\mathrm{H}(7)$ & 126.4 \\
\hline $\mathrm{C}(6)-\mathrm{C}(7)-\mathrm{H}(7)$ & 126.4 \\
\hline $\mathrm{C}(7)-\mathrm{C}(8)-\mathrm{C}(9)$ & $107.2(2)$ \\
\hline
\end{tabular}




\begin{tabular}{|c|c|}
\hline $\mathrm{C}(7)-\mathrm{C}(8)-\mathrm{H}(8)$ & 126.4 \\
\hline $\mathrm{C}(9)-\mathrm{C}(8)-\mathrm{H}(8)$ & 126.4 \\
\hline$N(2)-C(9)-C(10)$ & $125.4(2)$ \\
\hline $\mathrm{N}(2)-\mathrm{C}(9)-\mathrm{C}(8)$ & $109.82(19)$ \\
\hline$C(10)-C(9)-C(8)$ & $124.8(2)$ \\
\hline$C(9)-C(10)-C(11)$ & $124.0(2)$ \\
\hline$C(9)-C(10)-C(27)$ & $117.48(19)$ \\
\hline$C(11)-C(10)-C(27)$ & 118.55(19) \\
\hline$N(3)-C(11)-C(10)$ & $125.5(2)$ \\
\hline$N(3)-C(11)-C(12)$ & 109.56(19) \\
\hline$C(10)-C(11)-C(12)$ & $124.9(2)$ \\
\hline$C(13)-C(12)-C(11)$ & $107.4(2)$ \\
\hline $\mathrm{C}(13)-\mathrm{C}(12)-\mathrm{H}(12)$ & 126.3 \\
\hline $\mathrm{C}(11)-\mathrm{C}(12)-\mathrm{H}(12)$ & 126.3 \\
\hline$C(12)-C(13)-C(14)$ & $107.8(2)$ \\
\hline $\mathrm{C}(12)-\mathrm{C}(13)-\mathrm{H}(13)$ & 126.1 \\
\hline $\mathrm{C}(14)-\mathrm{C}(13)-\mathrm{H}(13)$ & 126.1 \\
\hline$N(3)-C(14)-C(15)$ & $126.0(2)$ \\
\hline$N(3)-C(14)-C(13)$ & 109.44(19) \\
\hline$C(15)-C(14)-C(13)$ & $124.6(2)$ \\
\hline$C(14)-C(15)-C(16)$ & $124.3(2)$ \\
\hline$C(14)-C(15)-C(33)$ & 117.57(19) \\
\hline$C(16)-C(15)-C(33)$ & 118.17(19) \\
\hline $\mathrm{N}(4)-\mathrm{C}(16)-\mathrm{C}(15)$ & $125.5(2)$ \\
\hline $\mathrm{N}(4)-\mathrm{C}(16)-\mathrm{C}(17)$ & $110.20(19)$ \\
\hline$C(15)-C(16)-C(17)$ & $124.3(2)$ \\
\hline$C(18)-C(17)-C(16)$ & $106.9(2)$ \\
\hline $\mathrm{C}(18)-\mathrm{C}(17)-\mathrm{H}(17)$ & 126.6 \\
\hline $\mathrm{C}(16)-\mathrm{C}(17)-\mathrm{H}(17)$ & 126.6 \\
\hline$C(17)-C(18)-C(19)$ & $107.5(2)$ \\
\hline $\mathrm{C}(17)-\mathrm{C}(18)-\mathrm{H}(18)$ & 126.2 \\
\hline $\mathrm{C}(19)-\mathrm{C}(18)-\mathrm{H}(18)$ & 126.2 \\
\hline $\mathrm{N}(4)-\mathrm{C}(19)-\mathrm{C}(20)$ & $125.4(2)$ \\
\hline $\mathrm{N}(4)-\mathrm{C}(19)-\mathrm{C}(18)$ & 109.99(19) \\
\hline$C(20)-C(19)-C(18)$ & $124.6(2)$ \\
\hline$C(19)-C(20)-C(1)$ & $124.0(2)$ \\
\hline$C(19)-C(20)-C(39)$ & 118.86(19) \\
\hline$C(1)-C(20)-C(39)$ & 117.12(19) \\
\hline$C(26)-C(21)-C(22)$ & $119.6(2)$ \\
\hline$C(26)-C(21)-C(5)$ & $120.8(2)$ \\
\hline$C(22)-C(21)-C(5)$ & $119.4(2)$ \\
\hline$C(21)-C(22)-C(23)$ & $120.3(3)$ \\
\hline $\mathrm{C}(21)-\mathrm{C}(22)-\mathrm{H}(22)$ & 119.9 \\
\hline $\mathrm{C}(23)-\mathrm{C}(22)-\mathrm{H}(22)$ & 119.9 \\
\hline$C(24)-C(23)-C(22)$ & $119.7(3)$ \\
\hline
\end{tabular}




\begin{tabular}{|c|c|}
\hline $\mathrm{C}(24)-\mathrm{C}(23)-\mathrm{H}(23)$ & 120.1 \\
\hline $\mathrm{C}(22)-\mathrm{C}(23)-\mathrm{H}(23)$ & 120.1 \\
\hline$C(23)-C(24)-C(25)$ & $120.3(2)$ \\
\hline $\mathrm{C}(23)-\mathrm{C}(24)-\mathrm{H}(24)$ & 119.8 \\
\hline $\mathrm{C}(25)-\mathrm{C}(24)-\mathrm{H}(24)$ & 119.8 \\
\hline$C(24)-C(25)-C(26)$ & $119.7(3)$ \\
\hline $\mathrm{C}(24)-\mathrm{C}(25)-\mathrm{H}(25)$ & 120.1 \\
\hline $\mathrm{C}(26)-\mathrm{C}(25)-\mathrm{H}(25)$ & 120.1 \\
\hline$C(21)-C(26)-C(25)$ & $120.3(3)$ \\
\hline $\mathrm{C}(21)-\mathrm{C}(26)-\mathrm{H}(26)$ & 119.8 \\
\hline $\mathrm{C}(25)-\mathrm{C}(26)-\mathrm{H}(26)$ & 119.8 \\
\hline $\mathrm{C}(28)-\mathrm{C}(27)-\mathrm{C}(32)$ & $118.7(2)$ \\
\hline $\mathrm{C}(28)-\mathrm{C}(27)-\mathrm{C}(10)$ & $119.7(2)$ \\
\hline $\mathrm{C}(32)-\mathrm{C}(27)-\mathrm{C}(10)$ & $121.6(2)$ \\
\hline$C(27)-C(28)-C(29)$ & $121.1(2)$ \\
\hline $\mathrm{C}(27)-\mathrm{C}(28)-\mathrm{H}(28)$ & 119.5 \\
\hline $\mathrm{C}(29)-\mathrm{C}(28)-\mathrm{H}(28)$ & 119.5 \\
\hline$C(30)-C(29)-C(28)$ & $119.8(2)$ \\
\hline $\mathrm{C}(30)-\mathrm{C}(29)-\mathrm{H}(29)$ & 120.1 \\
\hline $\mathrm{C}(28)-\mathrm{C}(29)-\mathrm{H}(29)$ & 120.1 \\
\hline$C(31)-C(30)-C(29)$ & $119.4(2)$ \\
\hline $\mathrm{C}(31)-\mathrm{C}(30)-\mathrm{H}(30)$ & 120.3 \\
\hline $\mathrm{C}(29)-\mathrm{C}(30)-\mathrm{H}(30)$ & 120.3 \\
\hline$C(30)-C(31)-C(32)$ & $120.7(2)$ \\
\hline $\mathrm{C}(30)-\mathrm{C}(31)-\mathrm{H}(31)$ & 119.7 \\
\hline $\mathrm{C}(32)-\mathrm{C}(31)-\mathrm{H}(31)$ & 119.7 \\
\hline$C(31)-C(32)-C(27)$ & $120.3(2)$ \\
\hline $\mathrm{C}(31)-\mathrm{C}(32)-\mathrm{H}(32)$ & 119.9 \\
\hline $\mathrm{C}(27)-\mathrm{C}(32)-\mathrm{H}(32)$ & 119.9 \\
\hline$C(38)-C(33)-C(34)$ & $118.8(2)$ \\
\hline$C(38)-C(33)-C(15)$ & $120.9(2)$ \\
\hline$C(34)-C(33)-C(15)$ & $120.28(19)$ \\
\hline$C(35)-C(34)-C(33)$ & $120.3(2)$ \\
\hline $\mathrm{C}(35)-\mathrm{C}(34)-\mathrm{H}(34)$ & 119.9 \\
\hline $\mathrm{C}(33)-\mathrm{C}(34)-\mathrm{H}(34)$ & 119.9 \\
\hline$C(34)-C(35)-C(36)$ & $120.8(2)$ \\
\hline $\mathrm{C}(34)-\mathrm{C}(35)-\mathrm{H}(35)$ & 119.6 \\
\hline $\mathrm{C}(36)-\mathrm{C}(35)-\mathrm{H}(35)$ & 119.6 \\
\hline$C(35)-C(36)-C(37)$ & $119.3(2)$ \\
\hline $\mathrm{C}(35)-\mathrm{C}(36)-\mathrm{H}(36)$ & 120.4 \\
\hline $\mathrm{C}(37)-\mathrm{C}(36)-\mathrm{H}(36)$ & 120.4 \\
\hline$C(38)-C(37)-C(36)$ & $120.3(2)$ \\
\hline $\mathrm{C}(38)-\mathrm{C}(37)-\mathrm{H}(37)$ & 119.9 \\
\hline $\mathrm{C}(36)-\mathrm{C}(37)-\mathrm{H}(37)$ & 119.9 \\
\hline$C(37)-C(38)-C(33)$ & $120.7(2)$ \\
\hline
\end{tabular}




\begin{tabular}{|c|c|}
\hline $\mathrm{C}(37)-\mathrm{C}(38)-\mathrm{H}(38)$ & 119.7 \\
\hline $\mathrm{C}(33)-\mathrm{C}(38)-\mathrm{H}(38)$ & 119.7 \\
\hline$C(40)-C(39)-C(44)$ & $119.2(2)$ \\
\hline$C(40)-C(39)-C(20)$ & 120.8(2) \\
\hline$C(44)-C(39)-C(20)$ & $119.9(2)$ \\
\hline$C(39)-C(40)-C(41)$ & 120.2(2) \\
\hline $\mathrm{C}(39)-\mathrm{C}(40)-\mathrm{H}(40)$ & 119.9 \\
\hline $\mathrm{C}(41)-\mathrm{C}(40)-\mathrm{H}(40)$ & 119.9 \\
\hline$C(42)-C(41)-C(40)$ & $120.0(2)$ \\
\hline $\mathrm{C}(42)-\mathrm{C}(41)-\mathrm{H}(41)$ & 120.0 \\
\hline $\mathrm{C}(40)-\mathrm{C}(41)-\mathrm{H}(41)$ & 120.0 \\
\hline$C(41)-C(42)-C(43)$ & $120.2(2$ \\
\hline $\mathrm{C}(41)-\mathrm{C}(42)-\mathrm{H}(42)$ & 119.9 \\
\hline $\mathrm{C}(43)-\mathrm{C}(42)-\mathrm{H}(42)$ & 119.9 \\
\hline$C(44)-C(43)-C(42)$ & $119.7(2)$ \\
\hline $\mathrm{C}(44)-\mathrm{C}(43)-\mathrm{H}(43)$ & 120.1 \\
\hline $\mathrm{C}(42)-\mathrm{C}(43)-\mathrm{H}(43)$ & 120.1 \\
\hline$C(43)-C(44)-C(39)$ & $120.7(2)$ \\
\hline $\mathrm{C}(43)-\mathrm{C}(44)-\mathrm{H}(44)$ & 119.6 \\
\hline $\mathrm{C}(39)-\mathrm{C}(44)-\mathrm{H}(44)$ & 119.6 \\
\hline $\mathrm{O}(2)-\mathrm{C}(45)-\mathrm{O}(1)$ & $129.6(2$ \\
\hline $\mathrm{O}(2)-\mathrm{C}(45)-\mathrm{C}(46)$ & $119.3(2)$ \\
\hline $\mathrm{O}(1)-\mathrm{C}(45)-\mathrm{C}(46)$ & 111.1(2) \\
\hline$F(1)-C(46)-F(3)$ & $108.0(3$ \\
\hline$F(1)-C(46)-F(2)$ & $106.2(3$ \\
\hline$F(3)-C(46)-F(2)$ & $106.0(3$ \\
\hline$F(1)-C(46)-C(45)$ & $113.1(2)$ \\
\hline$F(3)-C(46)-C(45)$ & $112.2(2)$ \\
\hline$F(2)-C(46)-C(45)$ & $110.9(2$ \\
\hline $\mathrm{C}(6 \mathrm{~S})-\mathrm{C}(1 \mathrm{~S})-\mathrm{C}(2 \mathrm{~S})$ & $110.2(3$ \\
\hline $\mathrm{C}(6 \mathrm{~S})-\mathrm{C}(1 \mathrm{~S})-\mathrm{H}(1 \mathrm{~S} 1)$ & 109.6 \\
\hline $\mathrm{C}(2 \mathrm{~S})-\mathrm{C}(1 \mathrm{~S})-\mathrm{H}(1 \mathrm{~S} 1)$ & 109.6 \\
\hline $\mathrm{C}(6 \mathrm{~S})-\mathrm{C}(1 \mathrm{~S})-\mathrm{H}(1 \mathrm{~S} 2)$ & 109.6 \\
\hline $\mathrm{C}(2 \mathrm{~S})-\mathrm{C}(1 \mathrm{~S})-\mathrm{H}(1 \mathrm{~S} 2)$ & 109.6 \\
\hline $\mathrm{H}(1 \mathrm{~S} 1)-\mathrm{C}(1 \mathrm{~S})-\mathrm{H}(1 \mathrm{~S} 2)$ & 108.1 \\
\hline$C(3 S)-C(2 S)-C(1 S)$ & 111.1(3) \\
\hline $\mathrm{C}(3 \mathrm{~S})-\mathrm{C}(2 \mathrm{~S})-\mathrm{H}(2 \mathrm{~S} 1)$ & 109.4 \\
\hline $\mathrm{C}(1 \mathrm{~S})-\mathrm{C}(2 \mathrm{~S})-\mathrm{H}(2 \mathrm{~S} 1)$ & 109.4 \\
\hline $\mathrm{C}(3 \mathrm{~S})-\mathrm{C}(2 \mathrm{~S})-\mathrm{H}(2 \mathrm{~S} 2)$ & 109.4 \\
\hline $\mathrm{C}(1 \mathrm{~S})-\mathrm{C}(2 \mathrm{~S})-\mathrm{H}(2 \mathrm{~S} 2)$ & 109.4 \\
\hline $\mathrm{H}(2 \mathrm{~S} 1)-\mathrm{C}(2 \mathrm{~S})-\mathrm{H}(2 \mathrm{~S} 2)$ & 108.0 \\
\hline$C(2 S)-C(3 S)-C(4 S)$ & $111.3(2$ \\
\hline $\mathrm{C}(2 \mathrm{~S})-\mathrm{C}(3 \mathrm{~S})-\mathrm{H}(3 \mathrm{~S} 1$ & 109.4 \\
\hline $\mathrm{C}(4 \mathrm{~S})-\mathrm{C}(3 \mathrm{~S})-\mathrm{H}(3 \mathrm{~S} 1)$ & 109.4 \\
\hline $\mathrm{C}(2 \mathrm{~S})-\mathrm{C}(3 \mathrm{~S})-\mathrm{H}(3 \mathrm{~S} 2)$ & 109.4 \\
\hline
\end{tabular}




\begin{tabular}{|c|c|}
\hline$(4 S)-C(3 S)-H(3 S 2)$ & 109.4 \\
\hline $\mathrm{H}(3 \mathrm{~S} 1)-\mathrm{C}(3 \mathrm{~S})-\mathrm{H}(3 \mathrm{~S} 2)$ & 108.0 \\
\hline$C(3 S)-C(4 S)-C(5 S)$ & $110.6(3)$ \\
\hline $\mathrm{C}(3 \mathrm{~S})-\mathrm{C}(4 \mathrm{~S})-\mathrm{H}\left(4 \mathrm{~S}_{1}\right)$ & 109.5 \\
\hline $\mathrm{C}(5 \mathrm{~S})-\mathrm{C}(4 \mathrm{~S})-\mathrm{H}(4 \mathrm{~S} 1)$ & 109.5 \\
\hline $\mathrm{C}(3 \mathrm{~S})-\mathrm{C}(4 \mathrm{~S})-\mathrm{H}(4 \mathrm{~S} 2)$ & 109.5 \\
\hline $\mathrm{C}(5 \mathrm{~S})-\mathrm{C}(4 \mathrm{~S})-\mathrm{H}(4 \mathrm{~S} 2)$ & 109.5 \\
\hline $\mathrm{H}(4 \mathrm{~S} 1)-\mathrm{C}(4 \mathrm{~S})-\mathrm{H}(4 \mathrm{~S} 2)$ & 108.1 \\
\hline$C(6 S)-C(5 S)-C(4 S)$ & $112.0(3)$ \\
\hline $\mathrm{C}(6 \mathrm{~S})-\mathrm{C}(5 \mathrm{~S})-\mathrm{H}(5 \mathrm{~S} 1)$ & 109.2 \\
\hline $\mathrm{C}(4 \mathrm{~S})-\mathrm{C}(5 \mathrm{~S})-\mathrm{H}(5 \mathrm{~S} 1)$ & 109.2 \\
\hline $\mathrm{C}(6 \mathrm{~S})-\mathrm{C}(5 \mathrm{~S})-\mathrm{H}(5 \mathrm{~S} 2)$ & 109.2 \\
\hline $\mathrm{C}(4 \mathrm{~S})-\mathrm{C}(5 \mathrm{~S})-\mathrm{H}(5 \mathrm{~S} 2)$ & 109.2 \\
\hline $\mathrm{H}(5 \mathrm{~S} 1)-\mathrm{C}(5 \mathrm{~S})-\mathrm{H}(5 \mathrm{~S} 2)$ & 107.9 \\
\hline$C(5 S)-C(6 S)-C(1 S)$ & $110.5(3)$ \\
\hline $\mathrm{C}(5 \mathrm{~S})-\mathrm{C}(6 \mathrm{~S})-\mathrm{H}(6 \mathrm{SA})$ & 109.5 \\
\hline $\mathrm{C}(1 \mathrm{~S})-\mathrm{C}(6 \mathrm{~S})-\mathrm{H}(6 \mathrm{SA})$ & 109.5 \\
\hline $\mathrm{C}(5 \mathrm{~S})-\mathrm{C}(6 \mathrm{~S})-\mathrm{H}(6 \mathrm{SB})$ & 109.5 \\
\hline $\mathrm{C}(1 \mathrm{~S})-\mathrm{C}(6 \mathrm{~S})-\mathrm{H}(6 \mathrm{SB})$ & 109.5 \\
\hline $\mathrm{H}(6 S A)-\mathrm{C}(6 S)-\mathrm{H}(6 S B)$ & 108.1 \\
\hline
\end{tabular}

Symmetry transformations used to generate equivalent atoms: 
Table S8. Anisotropic displacement parameters $\left(\AA^{2} \times 10^{3}\right)$ for 1 . The anisotropic displacement factor exponent takes the form:

$-2 \pi^{2}\left[h^{2} a^{* 2} U 11+\ldots+2 h k a^{*} b^{*} U_{12}\right]$

\begin{tabular}{|c|c|c|c|c|c|c|}
\hline & $\mathrm{U}_{11}$ & $\mathrm{U}_{22}$ & $\mathrm{U}_{33}$ & $\mathrm{U}_{23}$ & $U_{13}$ & $U_{12}$ \\
\hline $\mathrm{Fe}(1)$ & $13(1)$ & $16(1)$ & 16(1) & $-5(1)$ & $0(1)$ & $-3(1)$ \\
\hline $\mathrm{Fe}\left(1^{\prime}\right)$ & 13(1) & $16(1)$ & $16(1)$ & $-5(1)$ & $0(1)$ & $-3(1)$ \\
\hline$F(1)$ & $47(1)$ & $125(2)$ & $94(2)$ & $-75(2)$ & $26(1)$ & $-57(1)$ \\
\hline$F(2)$ & $67(2)$ & 126(2) & $53(1)$ & $-24(1)$ & $-28(1)$ & $-30(1)$ \\
\hline$F(3)$ & $41(1)$ & $121(2)$ & $151(2)$ & $-120(2)$ & $4(1)$ & $-16(1)$ \\
\hline$O(1)$ & $20(1)$ & $31(1)$ & $28(1)$ & $-12(1)$ & 2(1) & $-9(1)$ \\
\hline$O(2)$ & $30(1)$ & $40(1)$ & $35(1)$ & $-16(1)$ & $7(1)$ & $-16(1)$ \\
\hline$N(1)$ & $16(1)$ & $18(1)$ & $18(1)$ & $-4(1)$ & $0(1)$ & $-4(1)$ \\
\hline$N(2)$ & $17(1)$ & $18(1)$ & $20(1)$ & $-7(1)$ & $-1(1)$ & $-4(1)$ \\
\hline$N(3)$ & $17(1)$ & 19(1) & $18(1)$ & $-7(1)$ & $-2(1)$ & $-4(1)$ \\
\hline$N(4)$ & $16(1)$ & 19(1) & $20(1)$ & $-7(1)$ & $-1(1)$ & $-3(1)$ \\
\hline$C(1)$ & $16(1)$ & $20(1)$ & $23(1)$ & $-7(1)$ & $-1(1)$ & $-3(1)$ \\
\hline$C(2)$ & 19(1) & $23(1)$ & $27(1)$ & $-4(1)$ & 1(1) & $-1(1)$ \\
\hline$C(3)$ & $21(1)$ & $26(1)$ & $28(1)$ & $-7(1)$ & $5(1)$ & $-2(1)$ \\
\hline$C(4)$ & 17(1) & 24(1) & $22(1)$ & $-8(1)$ & 2(1) & $-5(1)$ \\
\hline$C(5)$ & $18(1)$ & $21(1)$ & $21(1)$ & $-8(1)$ & 2(1) & $-5(1)$ \\
\hline$C(6)$ & $20(1)$ & 21(1) & 18(1) & $-7(1)$ & 1(1) & $-6(1)$ \\
\hline$C(7)$ & $24(1)$ & $23(1)$ & $21(1)$ & $-5(1)$ & 3(1) & $-7(1)$ \\
\hline$C(8)$ & $26(1)$ & 21(1) & $22(1)$ & $-6(1)$ & $-1(1)$ & $-5(1)$ \\
\hline$C(9)$ & $20(1)$ & $19(1)$ & $20(1)$ & $-6(1)$ & $-2(1)$ & $-4(1)$ \\
\hline C(10) & $20(1)$ & $18(1)$ & 19(1) & $-6(1)$ & $-5(1)$ & $-3(1)$ \\
\hline$C(11)$ & $17(1)$ & 19(1) & $23(1)$ & $-9(1)$ & $-2(1)$ & $-4(1)$ \\
\hline$C(12)$ & 17(1) & $23(1)$ & $26(1)$ & $-10(1)$ & $-1(1)$ & $-3(1)$ \\
\hline$C(13)$ & 17(1) & $23(1)$ & $24(1)$ & $-9(1)$ & $-1(1)$ & $-4(1)$ \\
\hline$C(14)$ & $17(1)$ & $20(1)$ & $21(1)$ & $-10(1)$ & $0(1)$ & $-5(1)$ \\
\hline$C(15)$ & 19(1) & 21(1) & $22(1)$ & $-10(1)$ & 1(1) & $-8(1)$ \\
\hline$C(16)$ & $18(1)$ & $20(1)$ & $20(1)$ & $-8(1)$ & $-1(1)$ & $-6(1)$ \\
\hline$C(17)$ & $22(1)$ & 23(1) & $22(1)$ & $-7(1)$ & $0(1)^{\prime}$ & $-7(1)$ \\
\hline$C(18)$ & $22(1)$ & $22(1)$ & $22(1)$ & $-4(1)$ & $-3(1)$ & $-6(1)$ \\
\hline$C(19)$ & 19(1) & 19(1) & $20(1)$ & $-5(1)$ & $-2(1)$ & $-6(1)$ \\
\hline$C(20)$ & 18(1) & 18(1) & $22(1)$ & $-6(1)$ & $-3(1)$ & $-3(1)$ \\
\hline$C(21)$ & $21(1)$ & 19(1) & $23(1)$ & $-3(1)$ & 4(1) & $-2(1)$ \\
\hline$C(22)$ & $27(1)$ & $30(1)$ & $36(2)$ & $-11(1)$ & $5(1)$ & $-8(1)$ \\
\hline$C(23)$ & $26(1)$ & $29(1)$ & $45(2)$ & $-6(1)$ & $14(1)$ & $-8(1)$ \\
\hline$C(24)$ & $38(2)$ & $30(1)$ & $31(2)$ & $0(1)$ & $17(1)$ & $-1(1)$ \\
\hline$C(25)$ & $49(2)$ & $40(2)$ & $23(1)$ & $-9(1)$ & $3(1)$ & $-3(1)$ \\
\hline$C(26)$ & $30(1)$ & $38(1)$ & $25(1)$ & $-8(1)$ & 1(1) & $-8(1)$ \\
\hline$C(27)$ & 19(1) & $20(1)$ & 21(1) & $-6(1)$ & $-1(1)$ & $-3(1)$ \\
\hline
\end{tabular}




$\begin{array}{lllllll}\mathrm{C}(28) & 37(2) & 23(1) & 33(1) & -12(1) & -16(1) & -2(1) \\ \mathrm{C}(29) & 39(2) & 32(1) & 33(2) & -10(1) & -17(1) & -2(1) \\ \mathrm{C}(30) & 26(1) & 21(1) & 31(1) & -2(1) & -7(1) & -1(1) \\ \mathrm{C}(31) & 22(1) & 20(1) & 34(1) & -10(1) & 0(1) & -4(1) \\ \mathrm{C}(32) & 22(1) & 22(1) & 23(1) & -7(1) & -3(1) & -5(1) \\ \mathrm{C}(33) & 17(1) & 21(1) & 18(1) & -6(1) & -1(1) & -3(1) \\ \mathrm{C}(34) & 24(1) & 21(1) & 20(1) & -4(1) & -3(1) & -9(1) \\ \mathrm{C}(35) & 27(1) & 26(1) & 17(1) & -8(1) & -3(1) & -6(1) \\ \mathrm{C}(36) & 22(1) & 29(1) & 21(1) & -6(1) & 2(1) & -4(1) \\ \mathrm{C}(37) & 22(1) & 28(1) & 40(2) & -11(1) & 8(1) & -12(1) \\ \mathrm{C}(38) & 25(1) & 27(1) & 35(1) & -17(1) & 6(1) & -12(1) \\ \mathrm{C}(39) & 15(1) & 21(1) & 21(1) & -3(1) & 3(1) & -4(1) \\ \mathrm{C}(40) & 18(1) & 23(1) & 26(1) & -5(1) & -1(1) & -6(1) \\ \mathrm{C}(41) & 17(1) & 31(1) & 23(1) & 0(1) & -3(1) & -5(1) \\ \mathrm{C}(42) & 20(1) & 25(1) & 26(1) & -1(1) & 5(1) & -1(1) \\ \mathrm{C}(43) & 30(1) & 24(1) & 26(1) & -8(1) & 4(1) & -5(1) \\ \mathrm{C}(44) & 21(1) & 24(1) & 24(1) & -6(1) & -3(1) & -2(1) \\ \mathrm{C}(45) & 21(1) & 24(1) & 34(1) & -11(1) & -2(1) & -5(1) \\ \mathrm{C}(46) & 28(2) & 67(2) & 58(2) & -43(2) & 8(1) & -19(1) \\ \mathrm{C}(1 \mathrm{~S}) & 48(2) & 62(2) & 52(2) & -30(2) & 8(2) & -27(2) \\ \mathrm{C}(2 \mathrm{~S}) & 41(2) & 38(2) & 46(2) & -14(1) & -6(1) & -4(1) \\ \mathrm{C}(3 \mathrm{~S}) & 49(2) & 37(1) & 33(2) & -11(1) & 2(1) & -15(1) \\ \mathrm{C}(4 \mathrm{~S}) & 38(2) & 45(2) & 39(2) & -7(1) & -7(1) & -9(1) \\ \mathrm{C}(5 \mathrm{~S}) & 59(2) & 53(2) & 50(2) & 10(2) & -16(2) & -16(2) \\ \mathrm{C}(6 \mathrm{~S}) & 65(2) & 51(2) & 40(2) & -5(1) & -2(2) & -30(2) \\ & & & & & & \end{array}$


Table S9. Hydrogen coordinates and isotropic displacement parameters for 1.

\begin{tabular}{|c|c|c|c|c|}
\hline & $x$ & $y$ & $z$ & $\mathrm{U}(\mathrm{eq})$ \\
\hline$\overline{\mathrm{H}(2)}$ & -0.1451 & 0.6679 & 0.7199 & 0.031 \\
\hline $\mathrm{H}(3)$ & -0.1530 & 0.5312 & 0.8587 & 0.033 \\
\hline $\mathrm{H}(7)$ & 0.1054 & 0.0808 & 1.0089 & 0.027 \\
\hline $\mathrm{H}(8)$ & 0.3030 & -0.0715 & 0.9771 & 0.028 \\
\hline $\mathrm{H}(12)$ & 0.6379 & -0.0769 & 0.7249 & 0.026 \\
\hline$H(13)$ & 0.6375 & 0.0524 & 0.5837 & 0.025 \\
\hline $\mathrm{H}(17)$ & 0.3371 & 0.4737 & 0.4171 & 0.027 \\
\hline $\mathrm{H}(18)$ & 0.1507 & 0.6333 & 0.4538 & 0.027 \\
\hline $\mathrm{H}(22)$ & -0.1900 & 0.2790 & 0.9158 & 0.037 \\
\hline $\mathrm{H}(23)$ & -0.3354 & 0.2990 & 1.0262 & 0.043 \\
\hline $\mathrm{H}(24)$ & -0.2847 & 0.3480 & 1.1364 & 0.047 \\
\hline $\mathrm{H}(25)$ & -0.0893 & 0.3770 & 1.1372 & 0.048 \\
\hline $\mathrm{H}(26)$ & 0.0569 & 0.3545 & 1.0275 & 0.038 \\
\hline $\mathrm{H}(28)$ & 0.5607 & -0.0478 & 0.9514 & 0.037 \\
\hline$H(29)$ & 0.6792 & -0.2349 & 1.0320 & 0.042 \\
\hline $\mathrm{H}(30)$ & 0.6996 & -0.4156 & 0.9988 & 0.034 \\
\hline $\mathrm{H}(31)$ & 0.6015 & -0.4069 & 0.8849 & 0.030 \\
\hline $\mathrm{H}(32)$ & 0.4789 & -0.2206 & 0.8062 & 0.027 \\
\hline $\mathrm{H}(34)$ & 0.4642 & 0.1153 & 0.4524 & 0.025 \\
\hline $\mathrm{H}(35)$ & 0.5986 & 0.0934 & 0.3385 & 0.028 \\
\hline $\mathrm{H}(36)$ & 0.7290 & 0.2169 & 0.2884 & 0.031 \\
\hline $\mathrm{H}(37)$ & 0.7207 & 0.3670 & 0.3519 & 0.035 \\
\hline $\mathrm{H}(38)$ & 0.5885 & 0.3879 & 0.4672 & 0.032 \\
\hline $\mathrm{H}(40)$ & -0.0952 & 0.6570 & 0.4920 & 0.028 \\
\hline $\mathrm{H}(41)$ & -0.2150 & 0.8562 & 0.4281 & 0.031 \\
\hline $\mathrm{H}(42)$ & -0.2068 & 1.0199 & 0.4710 & 0.033 \\
\hline $\mathrm{H}(43)$ & -0.0844 & 0.9852 & 0.5807 & 0.033 \\
\hline $\mathrm{H}(44)$ & 0.0337 & 0.7867 & 0.6452 & 0.029 \\
\hline $\mathrm{H}(1 \mathrm{~S} 1)$ & 0.7858 & 0.1303 & 0.8309 & 0.059 \\
\hline $\mathrm{H}(1 \mathrm{~S} 2)$ & 0.7055 & 0.0972 & 0.7755 & 0.059 \\
\hline $\mathrm{H}(2 \mathrm{~S} 1)$ & 0.8347 & 0.1509 & 0.6593 & 0.051 \\
\hline $\mathrm{H}(2 \mathrm{~S} 2)$ & 0.7780 & 0.2642 & 0.6983 & 0.051 \\
\hline $\mathrm{H}(3 \mathrm{~S} 1)$ & 0.9686 & 0.2154 & 0.7610 & 0.047 \\
\hline $\mathrm{H}(3 \mathrm{~S} 2)$ & 0.9971 & 0.2301 & 0.6651 & 0.047 \\
\hline $\mathrm{H}(4 \mathrm{~S} 1)$ & 1.1446 & 0.0525 & 0.7434 & 0.050 \\
\hline $\mathrm{H}(4 \mathrm{~S} 2)$ & 1.0684 & 0.0181 & 0.6862 & 0.050 \\
\hline $\mathrm{H}(5 \mathrm{~S} 1)$ & 1.0723 & -0.1164 & 0.8170 & 0.070 \\
\hline $\mathrm{H}(5 \mathrm{~S} 2)$ & 1.0158 & -0.0047 & 0.8574 & 0.070 \\
\hline $\mathrm{H}(6 \mathrm{SA})$ & 0.8537 & -0.0830 & 0.8510 & 0.060 \\
\hline $\mathrm{H}(6 \mathrm{SB})$ & 0.8812 & -0.0666 & 0.7548 & 0.060 \\
\hline
\end{tabular}


Table S10. Torsion angles $\left[{ }^{\circ}\right]$ for 1.

\begin{tabular}{ll}
\hline $\mathrm{N}(3)-\mathrm{Fe}(1)-\mathrm{O}(1)-\mathrm{C}(45)$ & $41.2(2)$ \\
$\mathrm{N}(4)-\mathrm{Fe}(1)-\mathrm{O}(1)-\mathrm{C}(45)$ & $-50.5(2)$ \\
$\mathrm{N}(1)-\mathrm{Fe}(1)-\mathrm{O}(1)-\mathrm{C}(45)$ & $-140.83(19)$ \\
$\mathrm{N}(2)-\mathrm{Fe}(1)-\mathrm{O}(1)-\mathrm{C}(45)$ & $130.21(19)$ \\
$\mathrm{N}(2)-\mathrm{Fe}\left(1^{\prime}\right)-\mathrm{N}(1)-\mathrm{C}(4)$ & $-31.8(3)$ \\
$\mathrm{N}(3)-\mathrm{Fe}\left(1^{\prime}\right)-\mathrm{N}(1)-\mathrm{C}(4)$ & $-118.4(5)$ \\
$\mathrm{N}(4)-\mathrm{Fe}\left(1^{\prime}\right)-\mathrm{N}(1)-\mathrm{C}(4)$ & $179.56(18)$ \\
$\mathrm{N}(2)-\mathrm{Fe}\left(1^{\prime}\right)-\mathrm{N}(1)-\mathrm{C}(1)$ & $176.92(19)$ \\
$\mathrm{N}(3)-\mathrm{Fe}\left(1^{\prime}\right)-\mathrm{N}(1)-\mathrm{C}(1)$ & $90.3(6)$ \\
$\mathrm{N}(4)-\mathrm{Fe}\left(1^{\prime}\right)-\mathrm{N}(1)-\mathrm{C}(1)$ & $28.3(3)$ \\
$\mathrm{O}(1)-\mathrm{Fe}(1)-\mathrm{N}(1)-\mathrm{C}(4)$ & $-81.51(18)$ \\
$\mathrm{N}(3)-\mathrm{Fe}(1)-\mathrm{N}(1)-\mathrm{C}(4)$ & $93.5(2)$ \\
$\mathrm{N}(4)-\mathrm{Fe}(1)-\mathrm{N}(1)-\mathrm{C}(4)$ & $172.84(18)$ \\
$\mathrm{N}(2)-\mathrm{Fe}(1)-\mathrm{N}(1)-\mathrm{C}(4)$ & $17.04(18)$ \\
$\mathrm{O}(1)-\mathrm{Fe}(1)-\mathrm{N}(1)-\mathrm{C}(1)$ & $85.06(18)$ \\
$\mathrm{N}(3)-\mathrm{Fe}(1)-\mathrm{N}(1)-\mathrm{C}(1)$ & $-99.9(2)$ \\
$\mathrm{N}(4)-\mathrm{Fe}(1)-\mathrm{N}(1)-\mathrm{C}(1)$ & $-20.59(18)$ \\
$\mathrm{N}(2)-\mathrm{Fe}(1)-\mathrm{N}(1)-\mathrm{C}(1)$ & $-176.39(18)$ \\
$\mathrm{N}(1)-\mathrm{Fe}\left(1^{\prime}\right)-\mathrm{N}(2)-\mathrm{C}(6)$ & $35.9(3)$ \\
$\mathrm{N}(3)-\mathrm{Fe}\left(1^{\prime}\right)-\mathrm{N}(2)-\mathrm{C}(6)$ & $-177.36(17)$ \\
$\mathrm{N}(4)-\mathrm{Fe}\left(1^{\prime}\right)-\mathrm{N}(2)-\mathrm{C}(6)$ & $119.8(5)$ \\
$\mathrm{N}(1)-\mathrm{Fe}\left(1^{\prime}\right)-\mathrm{N}(2)-\mathrm{C}(9)$ & $-176.58(19)$ \\
$\mathrm{N}(3)-\mathrm{Fe}\left(1^{\prime}\right)-\mathrm{N}(2)-\mathrm{C}(9)$ & $-29.9(3)$ \\
$\mathrm{N}(4)-\mathrm{Fe}\left(1^{\prime}\right)-\mathrm{N}(2)-\mathrm{C}(9)$ & $-92.7(6)$ \\
$\mathrm{O}(1)-\mathrm{Fe}(1)-\mathrm{N}(2)-\mathrm{C}(6)$ & $87.97(18)$ \\
$\mathrm{N}(3)-\mathrm{Fe}(1)-\mathrm{N}(2)-\mathrm{C}(6)$ & $-170.11(19)$ \\
$\mathrm{N}(4)-\mathrm{Fe}(1)-\mathrm{N}(2)-\mathrm{C}(6)$ & $-90.5(2)$ \\
$\mathrm{N}(1)-\mathrm{Fe}(1)-\mathrm{N}(2)-\mathrm{C}(6)$ & $-12.68(18)$ \\
$\mathrm{O}(1)-\mathrm{Fe}(1)-\mathrm{N}(2)-\mathrm{C}(9)$ & $-82.94(18)$ \\
$\mathrm{N}(3)-\mathrm{Fe}(1)-\mathrm{N}(2)-\mathrm{C}(9)$ & $18.99(18)$ \\
$\mathrm{N}(4)-\mathrm{Fe}(1)-\mathrm{N}(2)-\mathrm{C}(9)$ & $98.6(2)$ \\
$\mathrm{N}(1)-\mathrm{Fe}(1)-\mathrm{N}(2)-\mathrm{C}(9)$ & $176.42(18)$ \\
$\mathrm{O}(1)-\mathrm{Fe}(1)-\mathrm{N}(3)-\mathrm{C}(11)$ & $71.61(18)$ \\
$\mathrm{N}(4)-\mathrm{Fe}(1)-\mathrm{N}(3)-\mathrm{C}(11)$ & $177.45(17)$ \\
$\mathrm{N}(1)-\mathrm{Fe}(1)-\mathrm{N}(3)-\mathrm{C}(11)$ & $-103.4(2)$ \\
$\mathrm{N}(2)-\mathrm{Fe}(1)-\mathrm{N}(3)-\mathrm{C}(11)$ & $-26.90(17)$ \\
$\mathrm{O}(1)-\mathrm{Fe}(1)-\mathrm{N}(3)-\mathrm{C}(14)$ & $-83.09(18)$ \\
$\mathrm{N}(4)-\mathrm{Fe}(1)-\mathrm{N}(3)-\mathrm{C}(14)$ & $22.75(17)$ \\
$\mathrm{N}(1)-\mathrm{Fe}(1)-\mathrm{N}(3)-\mathrm{C}(14)$ & $101.9(2)$ \\
$\mathrm{N}(2)-\mathrm{Fe}(1)-\mathrm{N}(3)-\mathrm{C}(14)$ & $178.40(18)$ \\
$\mathrm{N}(2)-\mathrm{Fe}\left(1^{\prime}\right)-\mathrm{N}(3)-\mathrm{C}(11)$ & $19.9(3)$ \\
$\mathrm{N}(1)-\mathrm{Fe}\left(1^{\prime}\right)-\mathrm{N}(3)-\mathrm{C}(11)$ & $108.5(5)$ \\
$\mathrm{N}(4)-\mathrm{Fe}\left(1^{\prime}\right)-\mathrm{N}(3)-\mathrm{C}(11)$ & $171.75(17)$ \\
& \\
& \\
& \\
& \\
&
\end{tabular}




\begin{tabular}{|c|c|}
\hline$N(2)-F e\left(1^{\prime}\right)-N(3)-C(14)$ & $-174.82(19)$ \\
\hline $\mathrm{N}(1)-\mathrm{Fe}\left(1^{\prime}\right)-\mathrm{N}(3)-\mathrm{C}(14)$ & $-86.2(6)$ \\
\hline $\mathrm{N}(4)-\mathrm{Fe}\left(1^{\prime}\right)-\mathrm{N}(3)-\mathrm{C}(14)$ & $-22.9(3)$ \\
\hline $\mathrm{O}(1)-\mathrm{Fe}(1)-\mathrm{N}(4)-\mathrm{C}(19)$ & $-80.75(18)$ \\
\hline $\mathrm{N}(3)-\mathrm{Fe}(1)-\mathrm{N}(4)-\mathrm{C}(19)$ & $177.06(18)$ \\
\hline $\mathrm{N}(1)-\mathrm{Fe}(1)-\mathrm{N}(4)-\mathrm{C}(19)$ & $19.87(18)$ \\
\hline $\mathrm{N}(2)-\mathrm{Fe}(1)-\mathrm{N}(4)-\mathrm{C}(19)$ & $97.7(2)$ \\
\hline $\mathrm{O}(1)-\mathrm{Fe}(1)-\mathrm{N}(4)-\mathrm{C}(16)$ & $83.73(18)$ \\
\hline $\mathrm{N}(3)-\mathrm{Fe}(1)-\mathrm{N}(4)-\mathrm{C}(16)$ & $-18.46(17)$ \\
\hline $\mathrm{N}(1)-\mathrm{Fe}(1)-\mathrm{N}(4)-\mathrm{C}(16)$ & $-175.65(18)$ \\
\hline$N(2)-F e(1)-N(4)-C(16)$ & $-97.8(2)$ \\
\hline $\mathrm{N}(2)-\mathrm{Fe}\left(1^{\prime}\right)-\mathrm{N}(4)-\mathrm{C}(19)$ & $-112.8(6)$ \\
\hline $\mathrm{N}(1)-\mathrm{Fe}\left(1^{\prime}\right)-\mathrm{N}(4)-\mathrm{C}(19)$ & $-26.6(3)$ \\
\hline $\mathrm{N}(3)-\mathrm{Fe}\left(1^{\prime}\right)-\mathrm{N}(4)-\mathrm{C}(19)$ & $-177.12(18)$ \\
\hline $\mathrm{N}(2)-\mathrm{Fe}\left(1^{\prime}\right)-\mathrm{N}(4)-\mathrm{C}(16)$ & $91.6(6)$ \\
\hline$N(1)-F e\left(1^{\prime}\right)-N(4)-C(16)$ & $177.87(19)$ \\
\hline $\mathrm{N}(3)-\mathrm{Fe}\left(1^{\prime}\right)-\mathrm{N}(4)-\mathrm{C}(16)$ & $27.3(3)$ \\
\hline$C(4)-N(1)-C(1)-C(20)$ & $-177.2(2)$ \\
\hline $\mathrm{Fe}\left(1^{\prime}\right)-\mathrm{N}(1)-\mathrm{C}(1)-\mathrm{C}(20)$ & $-22.9(4)$ \\
\hline $\mathrm{Fe}(1)-\mathrm{N}(1)-\mathrm{C}(1)-\mathrm{C}(20)$ & $14.0(3)^{\prime}$ \\
\hline $\mathrm{C}(4)-\mathrm{N}(1)-\mathrm{C}(1)-\mathrm{C}(2)$ & $0.5(2)$ \\
\hline $\mathrm{Fe}\left(1^{\prime}\right)-\mathrm{N}(1)-\mathrm{C}(1)-\mathrm{C}(2)$ & $154.8(3)$ \\
\hline $\mathrm{Fe}(1)-\mathrm{N}(1)-\mathrm{C}(1)-\mathrm{C}(2)$ & $-168.41(15)$ \\
\hline $\mathrm{N}(1)-\mathrm{C}(1)-\mathrm{C}(2)-\mathrm{C}(3)$ & $0.1(3)$ \\
\hline$C(20)-C(1)-C(2)-C(3)$ & $177.8(2)$ \\
\hline$C(1)-C(2)-C(3)-C(4)$ & $-0.6(3)$ \\
\hline$C(1)-N(1)-C(4)-C(5)$ & $176.9(2)$ \\
\hline $\mathrm{Fe}\left(1^{\prime}\right)-\mathrm{N}(1)-\mathrm{C}(4)-\mathrm{C}(5)$ & $19.6(4)$ \\
\hline $\mathrm{Fe}(1)-\mathrm{N}(1)-\mathrm{C}(4)-\mathrm{C}(5)$ & $-14.2(3)$ \\
\hline$C(1)-N(1)-C(4)-C(3)$ & $-0.9(2)$ \\
\hline $\mathrm{Fe}\left(1^{\prime}\right)-\mathrm{N}(1)-\mathrm{C}(4)-\mathrm{C}(3)$ & $-158.2(3)$ \\
\hline $\mathrm{Fe}(1)-\mathrm{N}(1)-\mathrm{C}(4)-\mathrm{C}(3)$ & $167.96(16)$ \\
\hline$C(2)-C(3)-C(4)-N(1)$ & $0.9(3)$ \\
\hline$C(2)-C(3)-C(4)-C(5)$ & $-176.9(2)$ \\
\hline$N(1)-C(4)-C(5)-C(6)$ & $-0.3(4)$ \\
\hline$C(3)-C(4)-C(5)-C(6)$ & $177.2(2)$ \\
\hline$N(1)-C(4)-C(5)-C(21)$ & $-179.1(2)$ \\
\hline$C(3)-C(4)-C(5)-C(21)$ & $-1.6(3)$ \\
\hline$C(9)-N(2)-C(6)-C(5)$ & $177.1(2)$ \\
\hline $\mathrm{Fe}\left(1^{\prime}\right)-\mathrm{N}(2)-\mathrm{C}(6)-\mathrm{C}(5)$ & $-28.9(4)$ \\
\hline $\mathrm{Fe}(1)-\mathrm{N}(2)-\mathrm{C}(6)-\mathrm{C}(5)$ & $4.7(3)$ \\
\hline$C(9)-N(2)-C(6)-C(7)$ & $-1.5(2)$ \\
\hline $\mathrm{Fe}\left(1^{\prime}\right)-\mathrm{N}(2)-\mathrm{C}(6)-\mathrm{C}(7)$ & $152.6(2)$ \\
\hline $\mathrm{Fe}(1)-\mathrm{N}(2)-\mathrm{C}(6)-\mathrm{C}(7)$ & $-173.90(15)$ \\
\hline$C(4)-C(5)-C(6)-N(2)$ & $5.4(4)$ \\
\hline
\end{tabular}




$\begin{array}{ll}\mathrm{C}(21)-\mathrm{C}(5)-\mathrm{C}(6)-\mathrm{N}(2) & -175.9(2) \\ \mathrm{C}(4)-\mathrm{C}(5)-\mathrm{C}(6)-\mathrm{C}(7) & -176.3(2) \\ \mathrm{C}(21)-\mathrm{C}(5)-\mathrm{C}(6)-\mathrm{C}(7) & 2.5(3) \\ \mathrm{N}(2)-\mathrm{C}(6)-\mathrm{C}(7)-\mathrm{C}(8) & 1.0(3) \\ \mathrm{C}(5)-\mathrm{C}(6)-\mathrm{C}(7)-\mathrm{C}(8) & -177.6(2) \\ \mathrm{C}(6)-\mathrm{C}(7)-\mathrm{C}(8)-\mathrm{C}(9) & -0.1(3) \\ \mathrm{C}(6)-\mathrm{N}(2)-\mathrm{C}(9)-\mathrm{C}(10) & -179.1(2) \\ \mathrm{Fe}\left(1^{\prime}\right)-\mathrm{N}(2)-\mathrm{C}(9)-\mathrm{C}(10) & 30.0(4) \\ \mathrm{Fe}(1)-\mathrm{N}(2)-\mathrm{C}(9)-\mathrm{C}(10) & -6.6(3) \\ \mathrm{C}(6)-\mathrm{N}(2)-\mathrm{C}(9)-\mathrm{C}(8) & 1.4(2) \\ \mathrm{Fe}(1)-\mathrm{N}(2)-\mathrm{C}(9)-\mathrm{C}(8) & -149.5(3) \\ \mathrm{Fe}(1)-\mathrm{N}(2)-\mathrm{C}(9)-\mathrm{C}(8) & 173.90(15) \\ \mathrm{C}(7)-\mathrm{C}(8)-\mathrm{C}(9)-\mathrm{N}(2) & -0.8(3) \\ \mathrm{C}(7)-\mathrm{C}(8)-\mathrm{C}(9)-\mathrm{C}(10) & 179.7(2) \\ \mathrm{N}(2)-\mathrm{C}(9)-\mathrm{C}(10)-\mathrm{C}(11) & -7.1(4) \\ \mathrm{C}(8)-\mathrm{C}(9)-\mathrm{C}(10)-\mathrm{C}(11) & 172.3(2) \\ \mathrm{N}(2)-\mathrm{C}(9)-\mathrm{C}(10)-\mathrm{C}(27) & 173.4(2) \\ \mathrm{C}(8)-\mathrm{C}(9)-\mathrm{C}(10)-\mathrm{C}(27) & -7.2(3) \\ \mathrm{C}(14)-\mathrm{N}(3)-\mathrm{C}(11)-\mathrm{C}(10) & -177.2(2) \\ \mathrm{Fe}(1)-\mathrm{N}(3)-\mathrm{C}(11)-\mathrm{C}(10) & 24.1(3) \\ \mathrm{Fe}(1)-\mathrm{N}(3)-\mathrm{C}(11)-\mathrm{C}(10) & -8.9(4) \\ \mathrm{C}(14)-\mathrm{N}(3)-\mathrm{C}(11)-\mathrm{C}(12) & 1.4(2) \\ \mathrm{Fe}(1)-\mathrm{N}(3)-\mathrm{C}(11)-\mathrm{C}(12) & -157.34(15) \\ \mathrm{Fe}(1)-\mathrm{N}(3)-\mathrm{C}(11)-\mathrm{C}(12) & 169.6(2) \\ \mathrm{C}(9)-\mathrm{C}(10)-\mathrm{C}(11)-\mathrm{N}(3) & -2.1(4) \\ \mathrm{C}(27)-\mathrm{C}(10)-\mathrm{C}(11)-\mathrm{N}(3) & 177.43(19) \\ \mathrm{C}(9)-\mathrm{C}(10)-\mathrm{C}(11)-\mathrm{C}(12) & 179.6(2) \\ \mathrm{C}(27)-\mathrm{C}(10)-\mathrm{C}(11)-\mathrm{C}(12) & -0.9(3) \\ \mathrm{N}(3)-\mathrm{C}(11)-\mathrm{C}(12)-\mathrm{C}(13) & -1.2(3) \\ \mathrm{C}(10)-\mathrm{C}(11)-\mathrm{C}(12)-\mathrm{C}(13) & 177.4(2) \\ \mathrm{C}(11)-\mathrm{C}(12)-\mathrm{C}(13)-\mathrm{C}(14) & 0.5(3) \\ \mathrm{C}(11)-\mathrm{N}(3)-\mathrm{C}(14)-\mathrm{C}(15) & -179.3(2) \\ \mathrm{Fe}(1)-\mathrm{N}(3)-\mathrm{C}(14)-\mathrm{C}(15) & -20.6(3) \\ \mathrm{Fe}(1))-\mathrm{N}(3)-\mathrm{C}(14)-\mathrm{C}(15) & 13.4(4) \\ \mathrm{C}(11)-\mathrm{N}(3)-\mathrm{C}(14)-\mathrm{C}(13) & -1.0(2) \\ \mathrm{Fe}(1)-\mathrm{N}(3)-\mathrm{C}(14)-\mathrm{C}(13) & 157.68(15) \\ \mathrm{Fe}(1)-\mathrm{N}(3)-\mathrm{C}(14)-\mathrm{C}(13) & -168.4(2) \\ \mathrm{C}(12)-\mathrm{C}(13)-\mathrm{C}(14)-\mathrm{N}(3) & 0.3(3) \\ \mathrm{C}(12)-\mathrm{C}(13)-\mathrm{C}(14)-\mathrm{C}(15) & 178.6(2) \\ \mathrm{N}(3)-\mathrm{C}(14)-\mathrm{C}(15)-\mathrm{C}(16) & 4.6(4) \\ \mathrm{C}(13)-\mathrm{C}(14)-\mathrm{C}(15)-\mathrm{C}(16) & -173.4(2) \\ \mathrm{N}(3)-\mathrm{C}(14)-\mathrm{C}(15)-\mathrm{C}(33) & -176.54(19) \\ \mathrm{C}(13)-\mathrm{C}(14)-\mathrm{C}(15)-\mathrm{C}(33) & 5.5(3) \\ \mathrm{C}(19)-\mathrm{N}(4)-\mathrm{C}(16)-\mathrm{C}(15) & 178.0(2) \\ \mathrm{Fe}(1)-\mathrm{N}(4)-\mathrm{C}(16)-\mathrm{C}(15) & 10.8(3) \\ & \\ & \end{array}$




\begin{tabular}{|c|c|}
\hline $\mathrm{Fe}\left(1^{\prime}\right)-\mathrm{N}(4)-\mathrm{C}(16)-\mathrm{C}(15)$ & $-23.1(4)$ \\
\hline $\mathrm{C}(19)-\mathrm{N}(4)-\mathrm{C}(16)-\mathrm{C}(17)$ & $-0.2(2)$ \\
\hline $\mathrm{Fe}(1)-\mathrm{N}(4)-\mathrm{C}(16)-\mathrm{C}(17)$ & $-167.40(15)$ \\
\hline $\mathrm{Fe}\left(1^{\prime}\right)-\mathrm{N}(4)-\mathrm{C}(16)-\mathrm{C}(17)$ & $158.6(3)$ \\
\hline $\mathrm{C}(14)-\mathrm{C}(15)-\mathrm{C}(16)-\mathrm{N}(4)$ & $0.7(4)$ \\
\hline$C(33)-C(15)-C(16)-N(4)$ & $-178.20(19)$ \\
\hline$C(14)-C(15)-C(16)-C(17)$ & $178.7(2)$ \\
\hline$C(33)-C(15)-C(16)-C(17)$ & $-0.2(3)$ \\
\hline$N(4)-C(16)-C(17)-C(18)$ & $0.7(3)$ \\
\hline$C(15)-C(16)-C(17)-C(18)$ & $-177.6(2)$ \\
\hline$C(16)-C(17)-C(18)-C(19)$ & $-0.9(3)$ \\
\hline$C(16)-N(4)-C(19)-C(20)$ & $-179.4(2)$ \\
\hline $\mathrm{Fe}(1)-\mathrm{N}(4)-\mathrm{C}(19)-\mathrm{C}(20)$ & $-12.3(3)$ \\
\hline $\mathrm{Fe}\left(1^{\prime}\right)-\mathrm{N}(4)-\mathrm{C}(19)-\mathrm{C}(20)$ & $20.4(3)$ \\
\hline $\mathrm{C}(16)-\mathrm{N}(4)-\mathrm{C}(19)-\mathrm{C}(18)$ & $-0.3(2)$ \\
\hline $\mathrm{Fe}(1)-\mathrm{N}(4)-\mathrm{C}(19)-\mathrm{C}(18)$ & $166.77(15)$ \\
\hline $\mathrm{Fe}\left(1^{\prime}\right)-\mathrm{N}(4)-\mathrm{C}(19)-\mathrm{C}(18)$ & $-160.5(2)$ \\
\hline $\mathrm{C}(17)-\mathrm{C}(18)-\mathrm{C}(19)-\mathrm{N}(4)$ & $0.8(3)$ \\
\hline$C(17)-C(18)-C(19)-C(20)$ & $179.9(2)$ \\
\hline$N(4)-C(19)-C(20)-C(1)$ & $-3.6(4)$ \\
\hline$C(18)-C(19)-C(20)-C(1)$ & $177.5(2)$ \\
\hline$N(4)-C(19)-C(20)-C(39)$ & $175.8(2)$ \\
\hline$C(18)-C(19)-C(20)-C(39)$ & $-3.1(3)$ \\
\hline$N(1)-C(1)-C(20)-C(19)$ & $2.7(4)$ \\
\hline$C(2)-C(1)-C(20)-C(19)$ & $-174.6(2)$ \\
\hline$N(1)-C(1)-C(20)-C(39)$ & $-176.7(2)$ \\
\hline$C(2)-C(1)-C(20)-C(39)$ & $5.9(3)$ \\
\hline$C(6)-C(5)-C(21)-C(26)$ & $82.5(3)$ \\
\hline$C(4)-C(5)-C(21)-C(26)$ & $-98.6(3)$ \\
\hline$C(6)-C(5)-C(21)-C(22)$ & $-101.2(3)$ \\
\hline$C(4)-C(5)-C(21)-C(22)$ & $77.7(3)$ \\
\hline$C(26)-C(21)-C(22)-C(23)$ & $-1.0(4)$ \\
\hline$C(5)-C(21)-C(22)-C(23)$ & $-177.4(2)$ \\
\hline$C(21)-C(22)-C(23)-C(24)$ & $0.7(4)$ \\
\hline$C(22)-C(23)-C(24)-C(25)$ & $0.0(4)$ \\
\hline$C(23)-C(24)-C(25)-C(26)$ & $-0.5(4)$ \\
\hline$C(22)-C(21)-C(26)-C(25)$ & $0.6(4)$ \\
\hline$C(5)-C(21)-C(26)-C(25)$ & $176.9(2)$ \\
\hline$C(24)-C(25)-C(26)-C(21)$ & $0.1(4)$ \\
\hline$C(9)-C(10)-C(27)-C(28)$ & $-66.9(3)$ \\
\hline$C(11)-C(10)-C(27)-C(28)$ & $113.6(3)$ \\
\hline$C(9)-C(10)-C(27)-C(32)$ & 111.1(3) \\
\hline$C(11)-C(10)-C(27)-C(32)$ & $-68.4(3)$ \\
\hline$C(32)-C(27)-C(28)-C(29)$ & $-1.7(4)$ \\
\hline$C(10)-C(27)-C(28)-C(29)$ & $176.4(2)$ \\
\hline
\end{tabular}




\begin{tabular}{|c|c|}
\hline$C(27)-C(28)-C(29)-C(30)$ & $1.2(4)$ \\
\hline$C(28)-C(29)-C(30)-C(31)$ & $0.2(4)$ \\
\hline$C(29)-C(30)-C(31)-C(32)$ & $-1.0(4)$ \\
\hline$C(30)-C(31)-C(32)-C(27)$ & $0.5(4)$ \\
\hline$C(28)-C(27)-C(32)-C(31)$ & $0.8(4)$ \\
\hline$C(10)-C(27)-C(32)-C(31)$ & $-177.2(2)$ \\
\hline$C(14)-C(15)-C(33)-C(38)$ & $-109.7(3)$ \\
\hline$C(16)-C(15)-C(33)-C(38)$ & $69.3(3)$ \\
\hline$C(14)-C(15)-C(33)-C(34)$ & $68.7(3)$ \\
\hline$C(16)-C(15)-C(33)-C(34)$ & $-112.4(2)$ \\
\hline$C(38)-C(33)-C(34)-C(35)$ & $0.3(3)$ \\
\hline$C(15)-C(33)-C(34)-C(35)$ & $-178.0(2)$ \\
\hline$C(33)-C(34)-C(35)-C(36)$ & $-0.1(3)$ \\
\hline$C(34)-C(35)-C(36)-C(37)$ & $-0.7(4)$ \\
\hline$C(35)-C(36)-C(37)-C(38)$ & $1.2(4)$ \\
\hline$C(36)-C(37)-C(38)-C(33)$ & $-0.9(4)$ \\
\hline$C(34)-C(33)-C(38)-C(37)$ & $0.1(4)$ \\
\hline$C(15)-C(33)-C(38)-C(37)$ & $178.5(2)$ \\
\hline$C(19)-C(20)-C(39)-C(40)$ & $66.9(3)$ \\
\hline$C(1)-C(20)-C(39)-C(40)$ & $-113.6(2)$ \\
\hline$C(19)-C(20)-C(39)-C(44)$ & $-114.3(2)$ \\
\hline$C(1)-C(20)-C(39)-C(44)$ & $65.2(3)$ \\
\hline$C(44)-C(39)-C(40)-C(41)$ & $0.6(3)$ \\
\hline$C(39)-C(40)-C(41)-C(42)$ & $0.4(3)$ \\
\hline$C(40)-C(41)-C(42)-C(43)$ & $-1.1(4)$ \\
\hline$C(41)-C(42)-C(43)-C(44)$ & $0.8(4)$ \\
\hline$C(42)-C(43)-C(44)-C(39)$ & $0.2(4)$ \\
\hline$C(40)-C(39)-C(44)-C(43)$ & $-0.9(3)$ \\
\hline$C(20)-C(39)-C(44)-C(43)$ & $-179.7(2)$ \\
\hline $\mathrm{Fe}(1)-\mathrm{O}(1)-\mathrm{C}(45)-\mathrm{O}(2)$ & $11.5(4)$ \\
\hline $\mathrm{Fe}(1)-\mathrm{O}(1)-\mathrm{C}(45)-\mathrm{C}(46)$ & $-168.30(18)$ \\
\hline $\mathrm{O}(2)-\mathrm{C}(45)-\mathrm{C}(46)-\mathrm{F}(1)$ & $7.5(4)$ \\
\hline $\mathrm{O}(1)-\mathrm{C}(45)-\mathrm{C}(46)-\mathrm{F}(1)$ & $-172.7(2)$ \\
\hline $\mathrm{O}(2)-\mathrm{C}(45)-\mathrm{C}(46)-\mathrm{F}(3)$ & $130.0(3)$ \\
\hline $\mathrm{O}(1)-\mathrm{C}(45)-\mathrm{C}(46)-\mathrm{F}(3)$ & $-50.2(4)$ \\
\hline $\mathrm{O}(2)-\mathrm{C}(45)-\mathrm{C}(46)-\mathrm{F}(2)$ & $-111.7(3)$ \\
\hline $\mathrm{O}(1)-\mathrm{C}(45)-\mathrm{C}(46)-\mathrm{F}(2)$ & $68.1(3)$ \\
\hline$C(6 S)-C(1 S)-C(2 S)-C(3 S)$ & $-57.1(3)$ \\
\hline$C(1 S)-C(2 S)-C(3 S)-C(4 S)$ & $56.6(3)$ \\
\hline$C(2 S)-C(3 S)-C(4 S)-C(5 S)$ & $-54.9(3)$ \\
\hline $\mathrm{C}(3 \mathrm{~S})-\mathrm{C}(4 \mathrm{~S})-\mathrm{C}(5 \mathrm{~S})-\mathrm{C}(6 \mathrm{~S})$ & $55.3(4)$ \\
\hline$C(4 S)-C(5 S)-C(6 S)-C(1 S)$ & $-56.3(4)$ \\
\hline $\mathrm{C}(2 \mathrm{~S})-\mathrm{C}(1 \mathrm{~S})-\mathrm{C}(6 \mathrm{~S})-\mathrm{C}(5 \mathrm{~S})$ & $56.7(4)$ \\
\hline
\end{tabular}

Symmetry transformations used to generate equivalent atoms: 
Part C: X-ray determination of (TPP)Fe(NO) $\left(\mathrm{OC}(=\mathrm{O}) \mathrm{CF}_{3}\right)(2)$

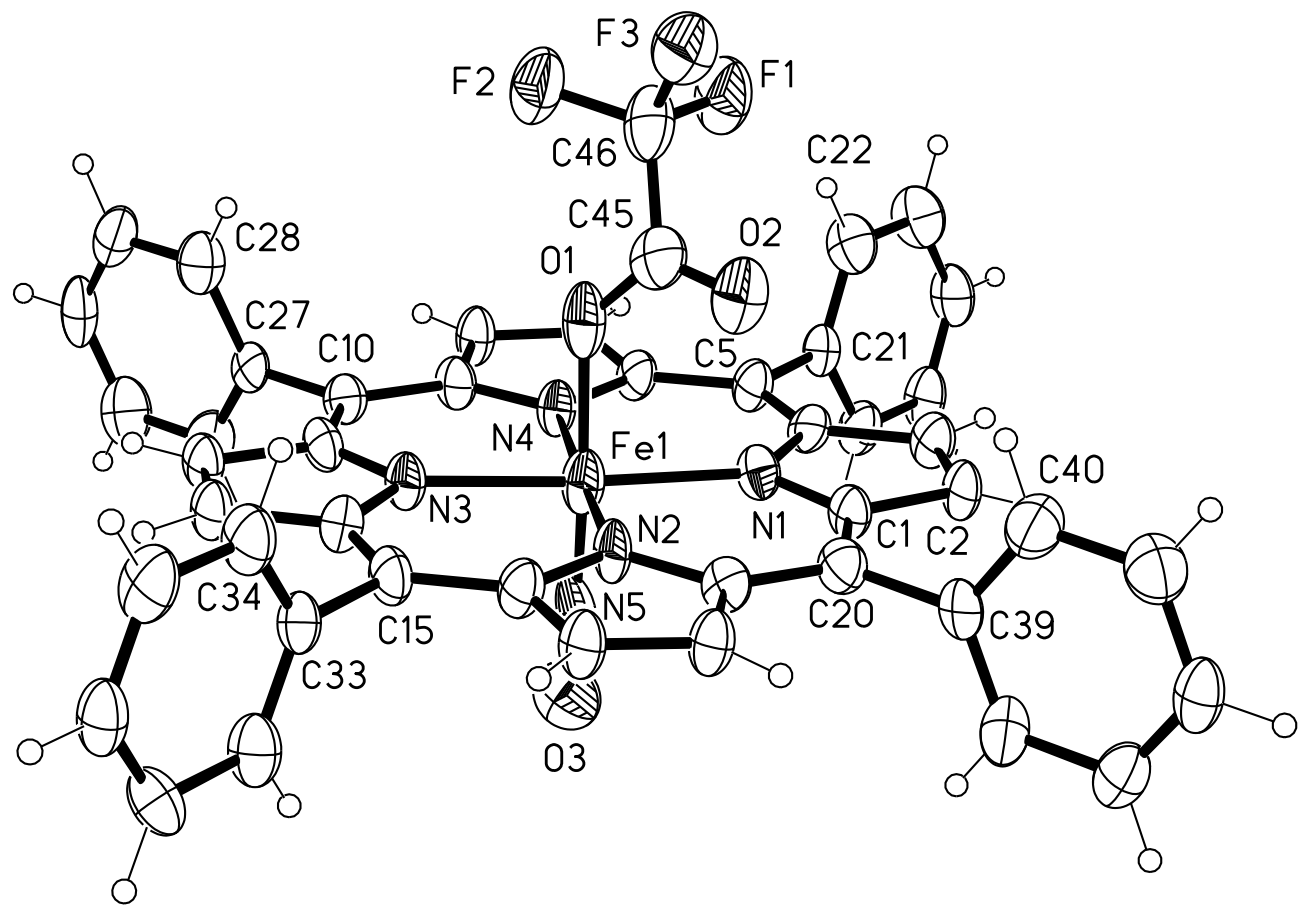

Figure S4. Molecular structure of (TPP) $\mathrm{Fe}(\mathrm{NO})\left(\mathrm{OC}(=\mathrm{O}) \mathrm{CF}_{3}\right)(2)$. 


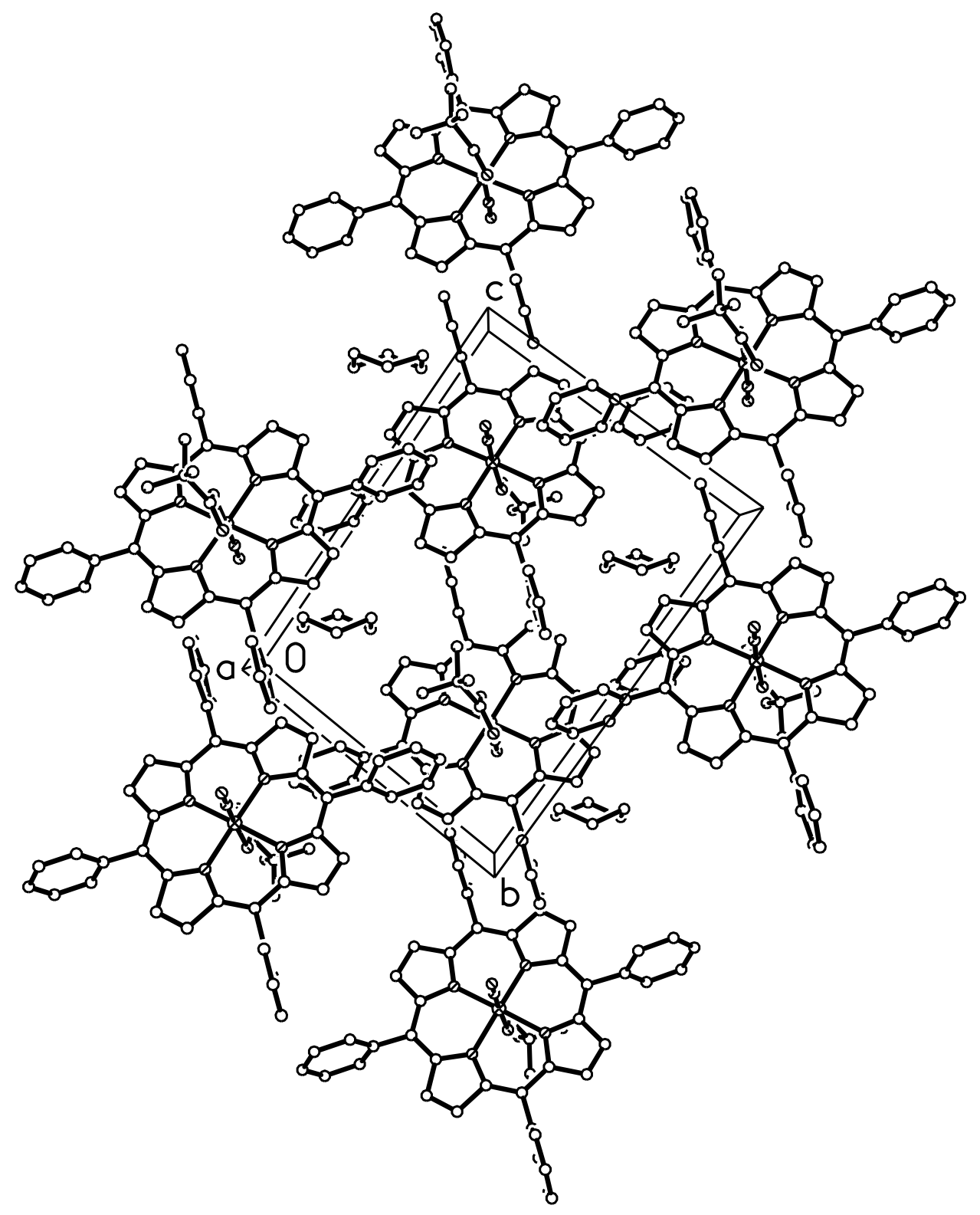

Figure S5. Crystal packing of (TPP) $\mathrm{Fe}(\mathrm{NO})\left(\mathrm{OC}(=\mathrm{O}) \mathrm{CF}_{3}\right)$ (2). 


\section{Comment}

The intensity data were truncated at $0.9 \AA$ because data in higher resolution shells had $\mathrm{R}$ (int) $>0.25$. The asymmetric unit of the cell contained one molecule of interest and one cyclohexane solvent molecule. The displacement ellipsoids were drawn at the 50\% probability level.

\section{Experimental}

A black block-shaped crystal of dimensions $0.42 \times 0.27 \times 0.25 \mathrm{~mm}$ was selected for structural analysis. Intensity data for this compound were collected using a diffractometer with a Bruker APEX ccd area detector (1) and graphite-monochromated Mo K $\alpha$ radiation ( $\lambda$ $=0.71073 \AA$ ). The sample was cooled to $100(2) \mathrm{K}$. Cell parameters were determined from a non-linear least squares fit of 9274 peaks in the range $2.53<\theta<27.34^{\circ}$. A total of 15943 data were measured in the range $2.07<\theta<23.25^{\circ}$ using $\omega$ oscillation frames. The data were corrected for absorption by the semi-empirical method (2) giving minimum and maximum transmission factors of 0.842 and 0.907 . The data were merged to form a set of 6115 independent data with $\mathrm{R}(\mathrm{int})=0.0565$ and a coverage of $99.5 \%$.

The triclinic space group $P \overline{1}$ was determined by statistical tests and verified by subsequent refinement. The structure was solved by direct methods and refined by fullmatrix least-squares methods on $F^{2}$ (3). Hydrogen atom positions were initially determined by geometry and refined by a riding model. Non-hydrogen atoms were refined with anisotropic displacement parameters. Hydrogen atom displacement parameters were set to 1.2 times the displacement parameters of the bonded atoms. A total of 577 parameters were refined against 0 restraints and 6115 data to give $\mathrm{wR}\left(F^{2}\right)=0.2494$ and $\mathrm{S}=1.095$ for weights of $\mathrm{w}=1 /\left[\sigma^{2}\left(F^{2}\right)+(0.0720 \mathrm{P})^{2}+13.8000 \mathrm{P}\right]$, where $\mathrm{P}=\left[F_{\mathrm{o}}{ }^{2}+2 F_{\mathrm{c}}{ }^{2}\right] / 3$. The final $\mathrm{R}(F)$ was 0.0812 for the 3525 observed, $[F>4 \sigma(F)]$, data. The largest shift/s.u. was 0.000 in the final refinement cycle. The final difference map had maxima and minima of 1.111 and $0.765 \mathrm{e} / \AA^{3}$, respectively. 


\section{References}

(1) (a) Data Collection: SMART Software Reference Manual (1998). Bruker-AXS, 5465 E. Cheryl Parkway, Madison, WI 53711-5373 USA. (b) Data Reduction: SAINT Software Reference Manual (1998). Bruker-AXS, 5465 E. Cheryl Parkway, Madison, WI 53711-5373, USA.

(2) G. M. Sheldrick (2002). SADABS. Program for Empirical Absorption Correction of Area Detector Data. University of Göttingen, Germany.

(3) (a) G. M. Sheldrick (2000). SHELXTL Version 6.10 Reference Manual. Bruker-AXS, 5465 E. Cheryl Parkway, Madison, WI 53711-5373 USA. (b) International Tables for Crystallography, Vol C, Tables 6.1.1.4, 4.2.6.8, and 4.2.4.2, Kluwer: Boston (1995). 
Table S11. Crystal data and structure refinement for $\mathbf{2} \cdot\left(\mathrm{C}_{6} \mathrm{H}_{12}\right)$

Empirical formula

Formula weight

Crystal system

Space group

Unit cell dimensions

Volume

Z, Z'

Density (calculated)

Wavelength

Temperature

$F(000)$

Absorption coefficient

Absorption correction

Max. and min. transmission

Theta range for data collection

Reflections collected

Independent reflections

Data / restraints / parameters

$w R\left(F^{2}\right.$ all data $)$

$R$ (F obsd data)

Goodness-of-fit on $F^{2}$

Observed data $[I>2 \sigma(I)]$

Largest and mean shift / s.u.

Largest diff. peak and hole
$\mathrm{C}_{46} \mathrm{H}_{28} \mathrm{~F}_{3} \mathrm{FeN}_{5} \mathrm{O}_{3} \cdot\left(\mathrm{C}_{6} \mathrm{H}_{12}\right)$

$\mathrm{C}_{52} \mathrm{H}_{40} \mathrm{~F}_{3} \mathrm{FeN}_{5} \mathrm{O}_{3}$

895.74

Triclinic

P1

$$
\begin{array}{ll}
a=11.055(9) \AA & \alpha=85.074(9)^{\circ} \\
b=13.204(10) \AA & \beta=72.130(9)^{\circ} \\
c=16.953(13) \AA & \gamma=65.693(8)^{\circ}
\end{array}
$$

2144(3) $\AA^{3}$

2, 1

$1.387 \mathrm{Mg} / \mathrm{m}^{3}$

$0.71073 \AA$

$100(2) \mathrm{K}$

928

$0.416 \mathrm{~mm}^{-1}$

Semi-empirical from equivalents

0.907 and 0.842

2.07 to $23.25^{\circ}$

15943

$6115[R$ (int) $=0.0565]$

6115 / 0 / 577

$w R 2=0.2494$

$R 1=0.0812$

1.095

3525

0.000 and 0.000

1.111 and $-0.765 \mathrm{e} / \AA^{3}$

$$
\begin{aligned}
& w R 2=\left\{\Sigma\left[w\left(F_{\mathrm{o}}^{2}-F_{\mathrm{c}}{ }^{2}\right)^{2}\right] / \Sigma\left[w\left(F_{\mathrm{o}}{ }^{2}\right)^{2}\right]\right\}^{1 / 2} \\
& R 1=\Sigma\left\|F_{\mathrm{o}}|-| F_{\mathrm{c}}\right\| / \Sigma\left|F_{\mathrm{o}}\right|
\end{aligned}
$$


Table S12. Atomic coordinates and equivalent isotropic displacement parameters for 2. $U(e q)$ is defined as one third of the trace of the orthogonalized $U_{i j}$ tensor.

\begin{tabular}{|c|c|c|c|c|}
\hline & $x$ & $y$ & $z$ & $\mathrm{U}(\mathrm{eq})$ \\
\hline $\mathrm{Fe}(1)$ & $0.51519(12)$ & $0.26891(9)$ & $0.74273(6)$ & $0.0387(4)$ \\
\hline$F(1)$ & $0.0539(5)$ & $0.4948(4)$ & $0.7773(3)$ & $0.0615(14)$ \\
\hline$F(2)$ & $0.1637(5)$ & $0.4903(4)$ & $0.6464(3)$ & $0.0600(13)$ \\
\hline$F(3)$ & $0.0300(5)$ & $0.4030(4)$ & $0.6889(3)$ & $0.0622(14)$ \\
\hline $\mathrm{O}(1)$ & $0.3544(6)$ & $0.3402(4)$ & $0.7068(3)$ & $0.0444(14)$ \\
\hline $\mathrm{O}(2)$ & $0.2191(6)$ & $0.2458(5)$ & $0.7520(3)$ & $0.0519(15)$ \\
\hline $\mathrm{O}(3)$ & $0.7527(7)$ & $0.1918(5)$ & $0.7925(4)$ & $0.0596(16)$ \\
\hline $\mathrm{N}(1)$ & $0.3934(6)$ & $0.2639(5)$ & $0.8572(3)$ & $0.0299(13)$ \\
\hline $\mathrm{N}(2)$ & $0.5346(6)$ & $0.1174(5)$ & $0.7151(3)$ & $0.0326(14)$ \\
\hline $\mathrm{N}(3)$ & $0.6265(6)$ & $0.2773(5)$ & $0.6255(3)$ & $0.0316(14)$ \\
\hline $\mathrm{N}(4)$ & $0.4857(6)$ & $0.4255(5)$ & $0.7681(3)$ & $0.0308(14)$ \\
\hline$N(5)$ & $0.6534(7)$ & $0.2204(5)$ & $0.7727(4)$ & $0.0412(16)$ \\
\hline$C(1)$ & $0.3621(7)$ & $0.1773(5)$ & $0.8907(4)$ & $0.0299(16)$ \\
\hline$C(2)$ & $0.2731(7)$ & $0.2064(6)$ & $0.9753(4)$ & $0.0338(17)$ \\
\hline$C(3)$ & $0.2536(7)$ & $0.3109(6)$ & $0.9920(4)$ & $0.0348(17)$ \\
\hline $\mathrm{C}(4)$ & $0.3279(7)$ & $0.3486(6)$ & $0.9188(4)$ & $0.0309(16)$ \\
\hline$C(5)$ & $0.3339(7)$ & $0.4516(6)$ & $0.9125(4)$ & $0.0331(17)$ \\
\hline$C(6)$ & $0.4073(7)$ & $0.4878(6)$ & $0.8413(4)$ & $0.0312(16)$ \\
\hline$C(7)$ & $0.4113(7)$ & $0.5943(6)$ & $0.8348(4)$ & $0.0362(18)$ \\
\hline $\mathrm{C}(8)$ & $0.4922(8)$ & $0.5974(6)$ & $0.7569(4)$ & $0.0367(18)$ \\
\hline $\mathrm{C}(9)$ & $0.5376(7)$ & $0.4913(6)$ & $0.7157(4)$ & $0.0308(16)$ \\
\hline$C(10)$ & $0.6222(7)$ & $0.4652(6)$ & $0.6324(4)$ & $0.0334(17)$ \\
\hline$C(11)$ & $0.6608(7)$ & $0.3637(6)$ & $0.5927(4)$ & $0.0324(17)$ \\
\hline$C(12)$ & $0.7454(7)$ & $0.3354(6)$ & $0.5066(4)$ & $0.0330(17)$ \\
\hline$C(13)$ & $0.7614(7)$ & $0.2329(6)$ & $0.4891(4)$ & $0.0341(17)$ \\
\hline$C(14)$ & $0.6878(7)$ & $0.1950(6)$ & $0.5635(4)$ & $0.0313(16)$ \\
\hline$C(15)$ & $0.6826(7)$ & $0.0919(6)$ & $0.5686(4)$ & $0.0342(17)$ \\
\hline$C(16)$ & $0.6101(7)$ & $0.0564(6)$ & $0.6401(4)$ & $0.0339(17)$ \\
\hline$C(17)$ & $0.6019(8)$ & $-0.0492(6)$ & $0.6475(4)$ & $0.0362(18)$ \\
\hline $\mathrm{C}(18)$ & $0.5263(7)$ & $-0.0545(6)$ & $0.7256(4)$ & $0.0338(17)$ \\
\hline $\mathrm{C}(19)$ & $0.4854(7)$ & $0.0488(5)$ & $0.7678(4)$ & $0.0290(16)$ \\
\hline$C(20)$ & $0.4031(7)$ & $0.0757(6)$ & $0.8517(4)$ & $0.0351(17)$ \\
\hline $\mathrm{C}(21)$ & $0.2582(7)$ & $0.5288(6)$ & $0.9880(4)$ & $0.0323(17)$ \\
\hline $\mathrm{C}(22)$ & $0.1267(8)$ & $0.6083(6)$ & $0.9983(4)$ & $0.043(2)$ \\
\hline $\mathrm{C}(23)$ & $0.0533(9)$ & $0.6765(7)$ & $1.0720(4)$ & $0.048(2)$ \\
\hline $\mathrm{C}(24)$ & $0.1142(8)$ & $0.6619(6)$ & $1.1343(4)$ & $0.043(2)$ \\
\hline$C(25)$ & $0.2457(9)$ & $0.5826(6)$ & $1.1253(4)$ & $0.043(2)$ \\
\hline$C(26)$ & $0.3183(8)$ & $0.5151(6)$ & $1.0518(4)$ & $0.0400(19)$ \\
\hline
\end{tabular}




\begin{tabular}{lllll}
$\mathrm{C}(27)$ & $0.6691(7)$ & $0.5491(6)$ & $0.5866(4)$ & $0.0291(16)$ \\
$\mathrm{C}(28)$ & $0.5957(9)$ & $0.6225(6)$ & $0.5384(4)$ & $0.045(2)$ \\
$\mathrm{C}(29)$ & $0.6407(9)$ & $0.7021(6)$ & $0.4958(4)$ & $0.045(2)$ \\
$\mathrm{C}(30)$ & $0.7592(9)$ & $0.7072(7)$ & $0.5000(4)$ & $0.047(2)$ \\
$\mathrm{C}(31)$ & $0.8333(10)$ & $0.6346(7)$ & $0.5476(5)$ & $0.056(2)$ \\
$\mathrm{C}(32)$ & $0.7891(9)$ & $0.5555(7)$ & $0.5902(5)$ & $0.051(2)$ \\
$\mathrm{C}(33)$ & $0.7574(7)$ & $0.0147(6)$ & $0.4940(4)$ & $0.0298(16)$ \\
$\mathrm{C}(34)$ & $0.6950(8)$ & $0.0159(6)$ & $0.4332(4)$ & $0.0418(19)$ \\
$\mathrm{C}(35)$ & $0.7677(9)$ & $-0.0531(6)$ & $0.3613(4)$ & $0.046(2)$ \\
$\mathrm{C}(36)$ & $0.9036(8)$ & $-0.1249(6)$ & $0.3500(4)$ & $0.0412(19)$ \\
$\mathrm{C}(37)$ & $0.9689(8)$ & $-0.1301(6)$ & $0.4090(4)$ & $0.0411(19)$ \\
$\mathrm{C}(38)$ & $0.8933(8)$ & $-0.0607(6)$ & $0.4812(4)$ & $0.0411(19)$ \\
$\mathrm{C}(39)$ & $0.3591(7)$ & $-0.0106(6)$ & $0.8988(4)$ & $0.0293(16)$ \\
$\mathrm{C}(40)$ & $0.2332(9)$ & $-0.0122(7)$ & $0.9035(5)$ & $0.045(2)$ \\
$\mathrm{C}(41)$ & $0.1922(9)$ & $-0.0916(7)$ & $0.9503(5)$ & $0.050(2)$ \\
$\mathrm{C}(42)$ & $0.2762(8)$ & $-0.1672(6)$ & $0.9925(4)$ & $0.0411(19)$ \\
$\mathrm{C}(43)$ & $0.4010(9)$ & $-0.1663(6)$ & $0.9876(4)$ & $0.044(2)$ \\
$\mathrm{C}(44)$ & $0.4426(9)$ & $-0.0879(7)$ & $0.9414(4)$ & $0.045(2)$ \\
$\mathrm{C}(45)$ & $0.2418(9)$ & $0.3261(7)$ & $0.7226(4)$ & $0.0421(19)$ \\
$\mathrm{C}(46)$ & $0.1246(9)$ & $0.4266(7)$ & $0.7076(4)$ & $0.049(2)$ \\
$\mathrm{C}(1 S)$ & $0.8333(10)$ & $0.9255(8)$ & $0.8085(5)$ & $0.066(3)$ \\
$\mathrm{C}(2 S)$ & $0.8373(10)$ & $0.8140(8)$ & $0.7873(5)$ & $0.069(3)$ \\
$\mathrm{C}(3 S)$ & $0.9697(10)$ & $0.7546(8)$ & $0.7144(5)$ & $0.063(3)$ \\
$\mathrm{C}(4 S)$ & $1.0977(9)$ & $0.7401(8)$ & $0.7373(5)$ & $0.059(2)$ \\
$\mathrm{C}(5 S)$ & $1.0936(10)$ & $0.8499(8)$ & $0.7593(6)$ & $0.069(3)$ \\
$\mathrm{C}(6 S)$ & $0.9583(10)$ & $0.9141(8)$ & $0.8294(6)$ & $0.067(3)$ \\
& & & & \\
\hline
\end{tabular}


Table S13. Bond lengths $[\AA]$ and angles $\left[{ }^{\circ}\right]$ for 2 .

\begin{tabular}{|c|c|c|c|}
\hline $\mathrm{Fe}(1)-\mathrm{N}(5)$ & $1.618(8)$ & $\mathrm{C}(13)-\mathrm{H}(13)$ & 0.9500 \\
\hline $\mathrm{Fe}(1)-\mathrm{O}(1)$ & $1.899(6)$ & $C(14)-C(15)$ & $1.381(10)$ \\
\hline $\mathrm{Fe}(1)-\mathrm{N}(2)$ & $2.004(6)$ & $C(15)-C(16)$ & $1.396(9)$ \\
\hline $\mathrm{Fe}(1)-\mathrm{N}(1)$ & $2.008(5)$ & $C(15)-C(33)$ & $1.486(9)$ \\
\hline $\mathrm{Fe}(1)-\mathrm{N}(3)$ & $2.013(5)$ & $C(16)-C(17)$ & $1.427(10)$ \\
\hline $\mathrm{Fe}(1)-\mathrm{N}(4)$ & $2.019(6)$ & $C(17)-C(18)$ & $1.346(9)$ \\
\hline$F(1)-C(46)$ & $1.361(9)$ & $\mathrm{C}(17)-\mathrm{H}(17)$ & 0.9500 \\
\hline $\mathrm{F}(2)-\mathrm{C}(46)$ & $1.358(9)$ & $C(18)-C(19)$ & $1.428(9)$ \\
\hline$F(3)-C(46)$ & $1.335(9)$ & $\mathrm{C}(18)-\mathrm{H}(18)$ & 0.9500 \\
\hline $\mathrm{O}(1)-\mathrm{C}(45)$ & $1.278(9)$ & $C(19)-C(20)$ & $1.416(9)$ \\
\hline $\mathrm{O}(2)-\mathrm{C}(45)$ & $1.220(9)$ & $C(20)-C(39)$ & $1.495(9)$ \\
\hline $\mathrm{O}(3)-\mathrm{N}(5)$ & $1.151(8)$ & $C(21)-C(22)$ & $1.365(10)$ \\
\hline$N(1)-C(1)$ & $1.358(8)$ & $C(21)-C(26)$ & 1.397(10) \\
\hline$N(1)-C(4)$ & $1.391(8)$ & $C(22)-C(23)$ & 1.414(10) \\
\hline $\mathrm{N}(2)-\mathrm{C}(19)$ & $1.372(8)$ & $\mathrm{C}(22)-\mathrm{H}(22)$ & 0.9500 \\
\hline $\mathrm{N}(2)-\mathrm{C}(16)$ & $1.390(8)$ & $C(23)-C(24)$ & $1.379(11)$ \\
\hline $\mathrm{N}(3)-\mathrm{C}(11)$ & $1.368(9)$ & $\mathrm{C}(23)-\mathrm{H}(23)$ & 0.9500 \\
\hline $\mathrm{N}(3)-\mathrm{C}(14)$ & $1.377(8)$ & $C(24)-C(25)$ & $1.368(11)$ \\
\hline$N(4)-C(9)$ & $1.355(8)$ & $\mathrm{C}(24)-\mathrm{H}(24)$ & 0.9500 \\
\hline$N(4)-C(6)$ & $1.377(8)$ & $C(25)-C(26)$ & $1.407(10)$ \\
\hline$C(1)-C(20)$ & $1.382(9)$ & $\mathrm{C}(25)-\mathrm{H}(25)$ & 0.9500 \\
\hline$C(1)-C(2)$ & $1.445(9)$ & $\mathrm{C}(26)-\mathrm{H}(26)$ & 0.9500 \\
\hline$C(2)-C(3)$ & $1.346(10)$ & $C(27)-C(28)$ & $1.375(10)$ \\
\hline $\mathrm{C}(2)-\mathrm{H}(2)$ & 0.9500 & $C(27)-C(32)$ & 1.382(11) \\
\hline$C(3)-C(4)$ & $1.437(9)$ & $C(28)-C(29)$ & 1.397(10) \\
\hline $\mathrm{C}(3)-\mathrm{H}(3)$ & 0.9500 & $\mathrm{C}(28)-\mathrm{H}(28)$ & 0.9500 \\
\hline$C(4)-C(5)$ & $1.382(10)$ & $C(29)-C(30)$ & $1.362(11)$ \\
\hline$C(5)-C(6)$ & $1.402(9)$ & $\mathrm{C}(29)-\mathrm{H}(29)$ & 0.9500 \\
\hline$C(5)-C(21)$ & $1.497(9)$ & $C(30)-C(31)$ & $1.366(12)$ \\
\hline $\mathrm{C}(6)-\mathrm{C}(7)$ & $1.419(10)$ & $\mathrm{C}(30)-\mathrm{H}(30)$ & 0.9500 \\
\hline$C(7)-C(8)$ & $1.357(9)$ & $C(31)-C(32)$ & $1.388(11)$ \\
\hline $\mathrm{C}(7)-\mathrm{H}(7)$ & 0.9500 & $\mathrm{C}(31)-\mathrm{H}(31)$ & 0.9500 \\
\hline$C(8)-C(9)$ & $1.442(9)$ & $\mathrm{C}(32)-\mathrm{H}(32)$ & 0.9500 \\
\hline $\mathrm{C}(8)-\mathrm{H}(8)$ & 0.9500 & $C(33)-C(38)$ & $1.379(10)$ \\
\hline$C(9)-C(10)$ & $1.411(9)$ & $C(33)-C(34)$ & 1.401(10) \\
\hline$C(10)-C(11)$ & 1.392(9) & $C(34)-C(35)$ & 1.396(10) \\
\hline$C(10)-C(27)$ & $1.477(10)$ & $\mathrm{C}(34)-\mathrm{H}(34)$ & 0.9500 \\
\hline$C(11)-C(12)$ & $1.454(9)$ & $C(35)-C(36)$ & $1.369(11)$ \\
\hline$C(12)-C(13)$ & $1.338(9)$ & $\mathrm{C}(35)-\mathrm{H}(35)$ & 0.9500 \\
\hline $\mathrm{C}(12)-\mathrm{H}(12)$ & 0.9500 & $C(36)-C(37)$ & $1.383(10)$ \\
\hline$C(13)-C(14)$ & $1.448(9)$ & $\mathrm{C}(36)-\mathrm{H}(36)$ & 0.9500 \\
\hline
\end{tabular}




\begin{tabular}{|c|c|c|c|}
\hline$C(37)-C(38)$ & $1.405(10)$ & $\mathrm{C}(1 \mathrm{~S})-\mathrm{H}(1 \mathrm{~S} 1)$ & 0.9900 \\
\hline $\mathrm{C}(37)-\mathrm{H}(37)$ & 0.9500 & $\mathrm{C}(1 \mathrm{~S})-\mathrm{H}(1 \mathrm{~S} 2)$ & 0.9900 \\
\hline $\mathrm{C}(38)-\mathrm{H}(38)$ & 0.9500 & $C(2 S)-C(3 S)$ & $1.548(12)$ \\
\hline$C(39)-C(40)$ & $1.379(10)$ & $\mathrm{C}(2 \mathrm{~S})-\mathrm{H}(2 \mathrm{~S} 1)$ & 0.9900 \\
\hline$C(39)-C(44)$ & 1.386(10) & $\mathrm{C}(2 \mathrm{~S})-\mathrm{H}(2 \mathrm{~S} 2)$ & 0.9900 \\
\hline$C(40)-C(41)$ & $1.400(11)$ & $C(3 S)-C(4 S)$ & $1.514(12)$ \\
\hline $\mathrm{C}(40)-\mathrm{H}(40)$ & 0.9500 & $\mathrm{C}(3 \mathrm{~S})-\mathrm{H}(3 \mathrm{~S} 1)$ & 0.9900 \\
\hline$C(41)-C(42)$ & $1.376(11)$ & $\mathrm{C}(3 \mathrm{~S})-\mathrm{H}(3 \mathrm{~S} 2)$ & 0.9900 \\
\hline $\mathrm{C}(41)-\mathrm{H}(41)$ & 0.9500 & $C(4 S)-C(5 S)$ & $1.508(12)$ \\
\hline$C(42)-C(43)$ & $1.362(11)$ & $\mathrm{C}(4 \mathrm{~S})-\mathrm{H}(4 \mathrm{~S} 1)$ & 0.9900 \\
\hline $\mathrm{C}(42)-\mathrm{H}(42)$ & 0.9500 & $\mathrm{C}(4 \mathrm{~S})-\mathrm{H}(4 \mathrm{~S} 2)$ & 0.9900 \\
\hline$C(43)-C(44)$ & $1.388(10)$ & $C(5 S)-C(6 S)$ & $1.551(12)$ \\
\hline $\mathrm{C}(43)-\mathrm{H}(43)$ & 0.9500 & $\mathrm{C}(5 \mathrm{~S})-\mathrm{H}(5 \mathrm{~S} 1)$ & 0.9900 \\
\hline $\mathrm{C}(44)-\mathrm{H}(44)$ & 0.9500 & $\mathrm{C}(5 \mathrm{~S})-\mathrm{H}(5 \mathrm{~S} 2)$ & 0.9900 \\
\hline$C(45)-C(46)$ & $1.494(12)$ & $\mathrm{C}(6 \mathrm{~S})-\mathrm{H}(6 \mathrm{~S} 1)$ & 0.9900 \\
\hline$C(1 S)-C(6 S)$ & $1.475(13)$ & $\mathrm{C}(6 \mathrm{~S})-\mathrm{H}(6 \mathrm{~S} 2)$ & 0.9900 \\
\hline$C(1 S)-C(2 S)$ & $1.525(13)$ & & \\
\hline $\mathrm{N}(5)-\mathrm{Fe}(1)-\mathrm{O}(1)$ & $174.1(3)$ & $\mathrm{C}(6)-\mathrm{N}(4)-\mathrm{Fe}(1)$ & $127.2(4)$ \\
\hline $\mathrm{N}(5)-\mathrm{Fe}(1)-\mathrm{N}(2)$ & $93.4(3)$ & $\mathrm{O}(3)-\mathrm{N}(5)-\mathrm{Fe}(1)$ & $175.8(6)$ \\
\hline $\mathrm{O}(1)-\mathrm{Fe}(1)-\mathrm{N}(2)$ & $92.2(2)$ & $N(1)-C(1)-C(20)$ & $126.9(6)$ \\
\hline $\mathrm{N}(5)-\mathrm{Fe}(1)-\mathrm{N}(1)$ & $92.2(3)$ & $N(1)-C(1)-C(2)$ & $110.2(6)$ \\
\hline $\mathrm{O}(1)-\mathrm{Fe}(1)-\mathrm{N}(1)$ & $89.6(2)$ & $C(20)-C(1)-C(2)$ & $122.8(6)$ \\
\hline $\mathrm{N}(2)-\mathrm{Fe}(1)-\mathrm{N}(1)$ & $89.6(2)$ & $C(3)-C(2)-C(1)$ & 106.6(6) \\
\hline $\mathrm{N}(5)-\mathrm{Fe}(1)-\mathrm{N}(3)$ & $91.4(3)$ & $\mathrm{C}(3)-\mathrm{C}(2)-\mathrm{H}(2)$ & 126.7 \\
\hline $\mathrm{O}(1)-\mathrm{Fe}(1)-\mathrm{N}(3)$ & $86.8(2)$ & $\mathrm{C}(1)-\mathrm{C}(2)-\mathrm{H}(2)$ & 126.7 \\
\hline $\mathrm{N}(2)-\mathrm{Fe}(1)-\mathrm{N}(3)$ & $90.0(2)$ & $C(2)-C(3)-C(4)$ & $108.0(6)$ \\
\hline $\mathrm{N}(1)-\mathrm{Fe}(1)-\mathrm{N}(3)$ & $176.4(2)$ & $\mathrm{C}(2)-\mathrm{C}(3)-\mathrm{H}(3)$ & 126.0 \\
\hline $\mathrm{N}(5)-\mathrm{Fe}(1)-\mathrm{N}(4)$ & $90.0(3)$ & $\mathrm{C}(4)-\mathrm{C}(3)-\mathrm{H}(3)$ & 126.0 \\
\hline $\mathrm{O}(1)-\mathrm{Fe}(1)-\mathrm{N}(4)$ & $84.3(2)$ & $C(5)-C(4)-N(1)$ & $126.4(6)$ \\
\hline $\mathrm{N}(2)-\mathrm{Fe}(1)-\mathrm{N}(4)$ & $176.6(2)$ & $C(5)-C(4)-C(3)$ & $124.9(6)$ \\
\hline $\mathrm{N}(1)-\mathrm{Fe}(1)-\mathrm{N}(4)$ & $90.4(2)$ & $N(1)-C(4)-C(3)$ & $108.7(6)$ \\
\hline $\mathrm{N}(3)-\mathrm{Fe}(1)-\mathrm{N}(4)$ & $89.7(2)$ & $C(4)-C(5)-C(6)$ & $124.8(6)$ \\
\hline $\mathrm{C}(45)-\mathrm{O}(1)-\mathrm{Fe}(1)$ & $132.7(5)$ & $C(4)-C(5)-C(21)$ & $117.3(6)$ \\
\hline$C(1)-N(1)-C(4)$ & $106.5(5)$ & $C(6)-C(5)-C(21)$ & $118.0(6)$ \\
\hline $\mathrm{C}(1)-\mathrm{N}(1)-\mathrm{Fe}(1)$ & $127.3(4)$ & $N(4)-C(6)-C(5)$ & $125.0(6)$ \\
\hline $\mathrm{C}(4)-\mathrm{N}(1)-\mathrm{Fe}(1)$ & $126.1(4)$ & $N(4)-C(6)-C(7)$ & $110.5(6)$ \\
\hline$C(19)-N(2)-C(16)$ & $105.6(5)$ & $C(5)-C(6)-C(7)$ & $124.5(6)$ \\
\hline $\mathrm{C}(19)-\mathrm{N}(2)-\mathrm{Fe}(1)$ & $127.2(4)$ & $C(8)-C(7)-C(6)$ & $106.7(6)$ \\
\hline $\mathrm{C}(16)-\mathrm{N}(2)-\mathrm{Fe}(1)$ & $127.0(4)$ & $\mathrm{C}(8)-\mathrm{C}(7)-\mathrm{H}(7)$ & 126.7 \\
\hline$C(11)-N(3)-C(14)$ & $106.6(5)$ & $\mathrm{C}(6)-\mathrm{C}(7)-\mathrm{H}(7)$ & 126.7 \\
\hline $\mathrm{C}(11)-\mathrm{N}(3)-\mathrm{Fe}(1)$ & $126.8(4)$ & $C(7)-C(8)-C(9)$ & $106.9(6)$ \\
\hline $\mathrm{C}(14)-\mathrm{N}(3)-\mathrm{Fe}(1)$ & $126.5(4)$ & $\mathrm{C}(7)-\mathrm{C}(8)-\mathrm{H}(8)$ & 126.6 \\
\hline $\mathrm{C}(9)-\mathrm{N}(4)-\mathrm{C}(6)$ & $106.1(5)$ & $\mathrm{C}(9)-\mathrm{C}(8)-\mathrm{H}(8)$ & 126.6 \\
\hline $\mathrm{C}(9)-\mathrm{N}(4)-\mathrm{Fe}(1)$ & $126.7(4)$ & $N(4)-C(9)-C(10)$ & $127.4(6)$ \\
\hline
\end{tabular}




\begin{tabular}{|c|c|c|c|}
\hline$N(4)-C(9)-C(8)$ & $109.9(5)$ & $\mathrm{C}(25)-\mathrm{C}(24)-\mathrm{H}(24)$ & 119.7 \\
\hline$C(10)-C(9)-C(8)$ & $122.7(6)$ & $\mathrm{C}(23)-\mathrm{C}(24)-\mathrm{H}(24)$ & 119.7 \\
\hline$C(11)-C(10)-C(9)$ & $121.9(6)$ & $C(24)-C(25)-C(26)$ & 119.3(7) \\
\hline$C(11)-C(10)-C(27)$ & 119.6(6) & $\mathrm{C}(24)-\mathrm{C}(25)-\mathrm{H}(25)$ & 120.4 \\
\hline$C(9)-C(10)-C(27)$ & $118.5(6)$ & $\mathrm{C}(26)-\mathrm{C}(25)-\mathrm{H}(25)$ & 120.4 \\
\hline$N(3)-C(11)-C(10)$ & 127.3(6) & $C(21)-C(26)-C(25)$ & $120.8(7)$ \\
\hline$N(3)-C(11)-C(12)$ & 109.5(6) & $\mathrm{C}(21)-\mathrm{C}(26)-\mathrm{H}(26)$ & 119.6 \\
\hline$C(10)-C(11)-C(12)$ & $123.2(6)$ & $\mathrm{C}(25)-\mathrm{C}(26)-\mathrm{H}(26)$ & 119.6 \\
\hline$C(13)-C(12)-C(11)$ & $107.0(6)$ & $C(28)-C(27)-C(32)$ & $118.0(7)$ \\
\hline $\mathrm{C}(13)-\mathrm{C}(12)-\mathrm{H}(12)$ & 126.5 & $C(28)-C(27)-C(10)$ & $121.0(7)$ \\
\hline $\mathrm{C}(11)-\mathrm{C}(12)-\mathrm{H}(12)$ & 126.5 & $C(32)-C(27)-C(10)$ & $121.0(6)$ \\
\hline$C(12)-C(13)-C(14)$ & 107.6(6) & $C(27)-C(28)-C(29)$ & $120.4(8)$ \\
\hline$C(12)-C(13)-H(13)$ & 126.2 & $\mathrm{C}(27)-\mathrm{C}(28)-\mathrm{H}(28)$ & 119.8 \\
\hline$C(14)-C(13)-H(13)$ & 126.2 & $\mathrm{C}(29)-\mathrm{C}(28)-\mathrm{H}(28)$ & 119.8 \\
\hline$N(3)-C(14)-C(15)$ & $127.1(6)$ & $C(30)-C(29)-C(28)$ & $120.8(8)$ \\
\hline$N(3)-C(14)-C(13)$ & 109.2(6) & $\mathrm{C}(30)-\mathrm{C}(29)-\mathrm{H}(29)$ & 119.6 \\
\hline$C(15)-C(14)-C(13)$ & $123.7(6)$ & $\mathrm{C}(28)-\mathrm{C}(29)-\mathrm{H}(29)$ & 119.6 \\
\hline$C(14)-C(15)-C(16)$ & $123.5(6)$ & $C(29)-C(30)-C(31)$ & $119.4(8)$ \\
\hline$C(14)-C(15)-C(33)$ & $118.4(6)$ & $\mathrm{C}(29)-\mathrm{C}(30)-\mathrm{H}(30)$ & 120.3 \\
\hline$C(16)-C(15)-C(33)$ & $118.2(6)$ & $\mathrm{C}(31)-\mathrm{C}(30)-\mathrm{H}(30)$ & 120.3 \\
\hline$N(2)-C(16)-C(15)$ & $125.9(6)$ & $C(30)-C(31)-C(32)$ & $120.1(8)$ \\
\hline$N(2)-C(16)-C(17)$ & 109.2(6) & $\mathrm{C}(30)-\mathrm{C}(31)-\mathrm{H}(31)$ & 119.9 \\
\hline$C(15)-C(16)-C(17)$ & $124.9(6)$ & $\mathrm{C}(32)-\mathrm{C}(31)-\mathrm{H}(31)$ & 119.9 \\
\hline$C(18)-C(17)-C(16)$ & 107.9(6) & $C(27)-C(32)-C(31)$ & $121.2(8)$ \\
\hline $\mathrm{C}(18)-\mathrm{C}(17)-\mathrm{H}(17)$ & 126.0 & $\mathrm{C}(27)-\mathrm{C}(32)-\mathrm{H}(32)$ & 119.4 \\
\hline $\mathrm{C}(16)-\mathrm{C}(17)-\mathrm{H}(17)$ & 126.0 & $\mathrm{C}(31)-\mathrm{C}(32)-\mathrm{H}(32)$ & 119.4 \\
\hline$C(17)-C(18)-C(19)$ & $106.9(6)$ & $C(38)-C(33)-C(34)$ & $117.0(6)$ \\
\hline $\mathrm{C}(17)-\mathrm{C}(18)-\mathrm{H}(18)$ & 126.6 & $C(38)-C(33)-C(15)$ & $120.8(6)$ \\
\hline $\mathrm{C}(19)-\mathrm{C}(18)-\mathrm{H}(18)$ & 126.6 & $C(34)-C(33)-C(15)$ & $122.1(7)$ \\
\hline$N(2)-C(19)-C(20)$ & $125.7(6)$ & $C(35)-C(34)-C(33)$ & $121.9(8)$ \\
\hline$N(2)-C(19)-C(18)$ & 110.3(5) & $\mathrm{C}(35)-\mathrm{C}(34)-\mathrm{H}(34)$ & 119.0 \\
\hline$C(20)-C(19)-C(18)$ & $123.9(6)$ & $\mathrm{C}(33)-\mathrm{C}(34)-\mathrm{H}(34)$ & 119.0 \\
\hline$C(1)-C(20)-C(19)$ & $122.9(6)$ & $C(36)-C(35)-C(34)$ & 119.2(7) \\
\hline$C(1)-C(20)-C(39)$ & 119.6(6) & $\mathrm{C}(36)-\mathrm{C}(35)-\mathrm{H}(35)$ & 120.4 \\
\hline$C(19)-C(20)-C(39)$ & $117.6(6)$ & $\mathrm{C}(34)-\mathrm{C}(35)-\mathrm{H}(35)$ & 120.4 \\
\hline$C(22)-C(21)-C(26)$ & $118.9(6)$ & $C(35)-C(36)-C(37)$ & $120.9(7)$ \\
\hline$C(22)-C(21)-C(5)$ & $121.0(6)$ & $\mathrm{C}(35)-\mathrm{C}(36)-\mathrm{H}(36)$ & 119.6 \\
\hline$C(26)-C(21)-C(5)$ & $119.9(7)$ & $\mathrm{C}(37)-\mathrm{C}(36)-\mathrm{H}(36)$ & 119.6 \\
\hline$C(21)-C(22)-C(23)$ & $120.5(7)$ & $C(36)-C(37)-C(38)$ & $119.0(7)$ \\
\hline $\mathrm{C}(21)-\mathrm{C}(22)-\mathrm{H}(22)$ & 119.7 & $\mathrm{C}(36)-\mathrm{C}(37)-\mathrm{H}(37)$ & 120.5 \\
\hline $\mathrm{C}(23)-\mathrm{C}(22)-\mathrm{H}(22)$ & 119.7 & $\mathrm{C}(38)-\mathrm{C}(37)-\mathrm{H}(37)$ & 120.5 \\
\hline$C(24)-C(23)-C(22)$ & 119.8(8) & $C(33)-C(38)-C(37)$ & $121.9(7)$ \\
\hline $\mathrm{C}(24)-\mathrm{C}(23)-\mathrm{H}(23)$ & 120.1 & $\mathrm{C}(33)-\mathrm{C}(38)-\mathrm{H}(38)$ & 119.0 \\
\hline $\mathrm{C}(22)-\mathrm{C}(23)-\mathrm{H}(23)$ & 120.1 & $\mathrm{C}(37)-\mathrm{C}(38)-\mathrm{H}(38)$ & 119.0 \\
\hline$C(25)-C(24)-C(23)$ & $120.7(7)$ & $C(40)-C(39)-C(44)$ & 118.9(7) \\
\hline
\end{tabular}




\begin{tabular}{|c|c|c|c|}
\hline$C(40)-C(39)-C(20)$ & $120.9(6)$ & $\mathrm{H}(1 \mathrm{~S} 1)-\mathrm{C}(1 \mathrm{~S})-\mathrm{H}(1 \mathrm{~S} 2)$ & 107.8 \\
\hline$C(44)-C(39)-C(20)$ & $120.1(6)$ & $C(1 S)-C(2 S)-C(3 S)$ & $108.9(8)$ \\
\hline$C(39)-C(40)-C(41)$ & $119.8(7)$ & $\mathrm{C}(1 \mathrm{~S})-\mathrm{C}(2 \mathrm{~S})-\mathrm{H}(2 \mathrm{~S} 1)$ & 109.9 \\
\hline $\mathrm{C}(39)-\mathrm{C}(40)-\mathrm{H}(40)$ & 120.1 & $\mathrm{C}(3 \mathrm{~S})-\mathrm{C}(2 \mathrm{~S})-\mathrm{H}(2 \mathrm{~S} 1)$ & 109.9 \\
\hline $\mathrm{C}(41)-\mathrm{C}(40)-\mathrm{H}(40)$ & 120.1 & $\mathrm{C}(1 \mathrm{~S})-\mathrm{C}(2 \mathrm{~S})-\mathrm{H}(2 \mathrm{~S} 2)$ & 109.9 \\
\hline$C(42)-C(41)-C(40)$ & $120.4(8)$ & $\mathrm{C}(3 \mathrm{~S})-\mathrm{C}(2 \mathrm{~S})-\mathrm{H}(2 \mathrm{~S} 2)$ & 109.9 \\
\hline $\mathrm{C}(42)-\mathrm{C}(41)-\mathrm{H}(41)$ & 119.8 & $\mathrm{H}(2 \mathrm{~S} 1)-\mathrm{C}(2 \mathrm{~S})-\mathrm{H}(2 \mathrm{~S} 2)$ & 108.3 \\
\hline $\mathrm{C}(40)-\mathrm{C}(41)-\mathrm{H}(41)$ & 119.8 & $C(4 S)-C(3 S)-C(2 S)$ & $110.0(7)$ \\
\hline$C(43)-C(42)-C(41)$ & $119.9(7)$ & $\mathrm{C}(4 \mathrm{~S})-\mathrm{C}(3 \mathrm{~S})-\mathrm{H}(3 \mathrm{~S} 1)$ & 109.7 \\
\hline $\mathrm{C}(43)-\mathrm{C}(42)-\mathrm{H}(42)$ & 120.1 & $\mathrm{C}(2 \mathrm{~S})-\mathrm{C}(3 \mathrm{~S})-\mathrm{H}(3 \mathrm{~S} 1)$ & 109.7 \\
\hline $\mathrm{C}(41)-\mathrm{C}(42)-\mathrm{H}(42)$ & 120.1 & $\mathrm{C}(4 \mathrm{~S})-\mathrm{C}(3 \mathrm{~S})-\mathrm{H}(3 \mathrm{~S} 2)$ & 109.7 \\
\hline$C(42)-C(43)-C(44)$ & $120.2(8)$ & $\mathrm{C}(2 \mathrm{~S})-\mathrm{C}(3 \mathrm{~S})-\mathrm{H}(3 \mathrm{~S} 2)$ & 109.7 \\
\hline $\mathrm{C}(42)-\mathrm{C}(43)-\mathrm{H}(43)$ & 119.9 & $\mathrm{H}(3 \mathrm{~S} 1)-\mathrm{C}(3 \mathrm{~S})-\mathrm{H}(3 \mathrm{~S} 2)$ & 108.2 \\
\hline $\mathrm{C}(44)-\mathrm{C}(43)-\mathrm{H}(43)$ & 119.9 & $C(5 S)-C(4 S)-C(3 S)$ & $111.9(8)$ \\
\hline$C(39)-C(44)-C(43)$ & $120.8(7)$ & $\mathrm{C}(5 \mathrm{~S})-\mathrm{C}(4 \mathrm{~S})-\mathrm{H}(4 \mathrm{~S} 1)$ & 109.2 \\
\hline $\mathrm{C}(39)-\mathrm{C}(44)-\mathrm{H}(44)$ & 119.6 & $\mathrm{C}(3 \mathrm{~S})-\mathrm{C}(4 \mathrm{~S})-\mathrm{H}(4 \mathrm{~S} 1)$ & 109.2 \\
\hline $\mathrm{C}(43)-\mathrm{C}(44)-\mathrm{H}(44)$ & 119.6 & $\mathrm{C}(5 \mathrm{~S})-\mathrm{C}(4 \mathrm{~S})-\mathrm{H}(4 \mathrm{~S} 2)$ & 109.2 \\
\hline $\mathrm{O}(2)-\mathrm{C}(45)-\mathrm{O}(1)$ & $128.1(8)$ & $\mathrm{C}(3 \mathrm{~S})-\mathrm{C}(4 \mathrm{~S})-\mathrm{H}(4 \mathrm{~S} 2)$ & 109.2 \\
\hline $\mathrm{O}(2)-\mathrm{C}(45)-\mathrm{C}(46)$ & 118.8(8) & $\mathrm{H}(4 \mathrm{~S} 1)-\mathrm{C}(4 \mathrm{~S})-\mathrm{H}(4 \mathrm{~S} 2)$ & 107.9 \\
\hline $\mathrm{O}(1)-\mathrm{C}(45)-\mathrm{C}(46)$ & $112.9(7)$ & $C(4 S)-C(5 S)-C(6 S)$ & $110.4(8)$ \\
\hline $\mathrm{F}(3)-\mathrm{C}(46)-\mathrm{F}(2)$ & $106.2(6)$ & $\mathrm{C}(4 \mathrm{~S})-\mathrm{C}(5 \mathrm{~S})-\mathrm{H}(5 \mathrm{~S} 1)$ & 109.6 \\
\hline $\mathrm{F}(3)-\mathrm{C}(46)-\mathrm{F}(1)$ & $105.5(7)$ & $\mathrm{C}(6 \mathrm{~S})-\mathrm{C}(5 \mathrm{~S})-\mathrm{H}(5 \mathrm{~S} 1)$ & 109.6 \\
\hline$F(2)-C(46)-F(1)$ & $105.9(6)$ & $\mathrm{C}(4 \mathrm{~S})-\mathrm{C}(5 \mathrm{~S})-\mathrm{H}(5 \mathrm{~S} 2)$ & 109.6 \\
\hline $\mathrm{F}(3)-\mathrm{C}(46)-\mathrm{C}(45)$ & $113.5(7)$ & $\mathrm{C}(6 \mathrm{~S})-\mathrm{C}(5 \mathrm{~S})-\mathrm{H}(5 \mathrm{~S} 2)$ & 109.6 \\
\hline$F(2)-C(46)-C(45)$ & $114.7(7)$ & $\mathrm{H}(5 \mathrm{~S} 1)-\mathrm{C}(5 \mathrm{~S})-\mathrm{H}(5 \mathrm{~S} 2)$ & 108.1 \\
\hline$F(1)-C(46)-C(45)$ & $110.4(6)$ & $C(1 S)-C(6 S)-C(5 S)$ & $111.8(8)$ \\
\hline$C(6 S)-C(1 S)-C(2 S)$ & $112.8(8)$ & $\mathrm{C}(1 \mathrm{~S})-\mathrm{C}(6 \mathrm{~S})-\mathrm{H}(6 \mathrm{~S} 1)$ & 109.3 \\
\hline $\mathrm{C}(6 \mathrm{~S})-\mathrm{C}(1 \mathrm{~S})-\mathrm{H}(1 \mathrm{~S} 1)$ & 109.0 & $\mathrm{C}(5 \mathrm{~S})-\mathrm{C}(6 \mathrm{~S})-\mathrm{H}(6 \mathrm{~S} 1)$ & 109.3 \\
\hline $\mathrm{C}(2 \mathrm{~S})-\mathrm{C}(1 \mathrm{~S})-\mathrm{H}(1 \mathrm{~S} 1)$ & 109.0 & $\mathrm{C}(1 \mathrm{~S})-\mathrm{C}(6 \mathrm{~S})-\mathrm{H}(6 \mathrm{~S} 2)$ & 109.3 \\
\hline $\mathrm{C}(6 \mathrm{~S})-\mathrm{C}(1 \mathrm{~S})-\mathrm{H}(1 \mathrm{~S} 2)$ & 109.0 & $\mathrm{C}(5 \mathrm{~S})-\mathrm{C}(6 \mathrm{~S})-\mathrm{H}(6 \mathrm{~S} 2)$ & 109.3 \\
\hline $\mathrm{C}(2 \mathrm{~S})-\mathrm{C}(1 \mathrm{~S})-\mathrm{H}(1 \mathrm{~S} 2)$ & 109.0 & $\mathrm{H}(6 \mathrm{~S} 1)-\mathrm{C}(6 \mathrm{~S})-\mathrm{H}(6 \mathrm{~S} 2)$ & 107.9 \\
\hline
\end{tabular}


Table S14. Anisotropic displacement parameters $\left(\AA^{2} \times 10^{3}\right)$ for 2 . The anisotropic displacement factor exponent takes the form:

$-2 \pi^{2}\left[h^{2} a^{* 2} U_{11}+\ldots+2 h k a^{*} b^{*} U_{12}\right]$

\begin{tabular}{|c|c|c|c|c|c|c|}
\hline & $U_{11}$ & $U_{22}$ & $U_{33}$ & $U_{23}$ & $U_{13}$ & $\mathrm{U}_{12}$ \\
\hline $\mathrm{Fe}(1)$ & $52(1)$ & $42(1)$ & $20(1)$ & $-5(1)$ & $0(1)$ & $-23(1)$ \\
\hline$F(1)$ & $74(3)$ & $51(3)$ & $36(2)$ & $-8(2)$ & $5(2)$ & $-16(3)$ \\
\hline$F(2)$ & $76(4)$ & $57(3)$ & $33(2)$ & $4(2)$ & $-3(2)$ & $-24(3)$ \\
\hline$F(3)$ & $63(3)$ & $76(4)$ & $47(3)$ & $7(2)$ & $-16(2)$ & $-28(3)$ \\
\hline$O(1)$ & $62(4)$ & $48(3)$ & $22(2)$ & $-7(2)$ & $5(2)$ & $-32(3)$ \\
\hline $\mathrm{O}(2)$ & $67(4)$ & $52(4)$ & $38(3)$ & $2(3)$ & $-8(3)$ & $-31(3)$ \\
\hline $\mathrm{O}(3)$ & $60(4)$ & $61(4)$ & $54(4)$ & $-14(3)$ & $-13(3)$ & $-21(3)$ \\
\hline $\mathrm{N}(1)$ & $35(3)$ & $37(3)$ & $18(3)$ & $-6(2)$ & $-4(2)$ & $-17(3)$ \\
\hline $\mathrm{N}(2)$ & $43(4)$ & $37(3)$ & $13(3)$ & $-5(2)$ & $3(2)$ & $-19(3)$ \\
\hline $\mathrm{N}(3)$ & $39(4)$ & $35(3)$ & $17(3)$ & $-5(2)$ & $-1(2)$ & $-16(3)$ \\
\hline $\mathrm{N}(4)$ & $41(4)$ & $36(3)$ & $16(3)$ & $-5(2)$ & $-2(2)$ & $-19(3)$ \\
\hline$N(5)$ & $45(4)$ & $49(4)$ & $27(3)$ & $-14(3)$ & $11(3)$ & $-29(4)$ \\
\hline$C(1)$ & $35(4)$ & $30(4)$ & $19(3)$ & $-5(3)$ & $-1(3)$ & $-12(3)$ \\
\hline$C(2)$ & $43(4)$ & $41(4)$ & $13(3)$ & $-3(3)$ & $2(3)$ & $-18(4)$ \\
\hline$C(3)$ & $43(5)$ & $37(4)$ & $20(3)$ & $-4(3)$ & $-3(3)$ & $-15(4)$ \\
\hline$C(4)$ & $36(4)$ & $37(4)$ & $17(3)$ & $2(3)$ & $-8(3)$ & $-12(3)$ \\
\hline$C(5)$ & $41(4)$ & $36(4)$ & $20(3)$ & $0(3)$ & $-5(3)$ & $-16(4)$ \\
\hline$C(6)$ & $39(4)$ & $34(4)$ & $15(3)$ & $-3(3)$ & $-4(3)$ & $-12(3)$ \\
\hline$C(7)$ & $45(5)$ & $33(4)$ & $22(4)$ & $-7(3)$ & $4(3)$ & $-16(4)$ \\
\hline $\mathrm{C}(8)$ & $49(5)$ & $37(4)$ & $22(4)$ & $-4(3)$ & $0(3)$ & $-22(4)$ \\
\hline $\mathrm{C}(9)$ & $37(4)$ & $32(4)$ & $19(3)$ & $-8(3)$ & $-1(3)$ & $-14(3)$ \\
\hline$C(10)$ & $36(4)$ & $37(4)$ & 21(3) & $-4(3)$ & $-5(3)$ & $-10(3)$ \\
\hline$C(11)$ & $31(4)$ & $40(4)$ & $21(3)$ & $-6(3)$ & $1(3)$ & $-13(3)$ \\
\hline$C(12)$ & $39(4)$ & $33(4)$ & 21(3) & $-6(3)$ & $0(3)$ & $-13(3)$ \\
\hline$C(13)$ & $42(4)$ & $36(4)$ & $19(3)$ & $-5(3)$ & $0(3)$ & $-16(4)$ \\
\hline$C(14)$ & $38(4)$ & $35(4)$ & $24(3)$ & $3(3)$ & $-7(3)$ & $-20(3)$ \\
\hline$C(15)$ & $36(4)$ & $40(4)$ & $20(3)$ & $-4(3)$ & $0(3)$ & $-13(4)$ \\
\hline$C(16)$ & $43(5)$ & $35(4)$ & 21(4) & $0(3)$ & $-5(3)$ & $-16(4)$ \\
\hline$C(17)$ & $48(5)$ & $43(4)$ & $16(3)$ & $-5(3)$ & $-2(3)$ & $-21(4)$ \\
\hline$C(18)$ & $46(5)$ & $38(4)$ & $19(3)$ & $-2(3)$ & $-4(3)$ & $-22(4)$ \\
\hline$C(19)$ & $36(4)$ & $35(4)$ & 18(3) & $-6(3)$ & $-8(3)$ & $-14(3)$ \\
\hline$C(20)$ & $34(4)$ & $46(5)$ & $26(4)$ & $2(3)$ & $-9(3)$ & $-17(4)$ \\
\hline $\mathrm{C}(21)$ & $37(4)$ & $38(4)$ & $22(3)$ & $2(3)$ & $-3(3)$ & $-19(4)$ \\
\hline$C(22)$ & $49(5)$ & $53(5)$ & $30(4)$ & $-9(4)$ & $-7(4)$ & $-24(4)$ \\
\hline$C(23)$ & $53(5)$ & $48(5)$ & $35(4)$ & $-16(4)$ & $-2(4)$ & $-18(4)$ \\
\hline$C(24)$ & $52(5)$ & 46(5) & $26(4)$ & $-13(3)$ & $4(4)$ & $-23(4)$ \\
\hline
\end{tabular}




\begin{tabular}{|c|c|c|c|c|c|c|}
\hline$C(25)$ & $62(6)$ & $50(5)$ & $17(3)$ & $-5(3)$ & $-3(3)$ & $-29(5)$ \\
\hline$C(26)$ & $50(5)$ & $40(4)$ & $25(4)$ & $0(3)$ & $-5(3)$ & $-17(4)$ \\
\hline$C(27)$ & $36(4)$ & $33(4)$ & $13(3)$ & $-8(3)$ & $-2(3)$ & $-10(3)$ \\
\hline$C(28)$ & $53(5)$ & $47(5)$ & $33(4)$ & $-2(4)$ & $-6(4)$ & $-24(4)$ \\
\hline$C(29)$ & $64(6)$ & $32(4)$ & $28(4)$ & $3(3)$ & $-4(4)$ & $-15(4)$ \\
\hline$C(30)$ & $66(6)$ & $43(5)$ & $25(4)$ & $-7(3)$ & $10(4)$ & $-31(5)$ \\
\hline$C(31)$ & $62(6)$ & $65(6)$ & $46(5)$ & $-1(5)$ & $-4(4)$ & $-39(5)$ \\
\hline$C(32)$ & $59(6)$ & $54(5)$ & $38(4)$ & $5(4)$ & $-10(4)$ & $-25(5)$ \\
\hline C(33) & $35(4)$ & $35(4)$ & $25(3)$ & $2(3)$ & $-4(3)$ & $-23(4)$ \\
\hline$C(34)$ & $51(5)$ & $43(5)$ & $24(4)$ & $-7(3)$ & $-3(3)$ & $-17(4)$ \\
\hline$C(35)$ & $66(6)$ & $47(5)$ & $23(4)$ & $-7(3)$ & $-11(4)$ & $-22(5)$ \\
\hline$C(36)$ & $56(6)$ & $39(4)$ & $25(4)$ & $-5(3)$ & $0(4)$ & $-23(4)$ \\
\hline$C(37)$ & $36(4)$ & $44(5)$ & $35(4)$ & $-14(3)$ & $1(3)$ & $-13(4)$ \\
\hline$C(38)$ & $53(5)$ & $45(5)$ & $28(4)$ & $-6(3)$ & $-4(3)$ & $-26(4)$ \\
\hline C(39) & $36(4)$ & $34(4)$ & $13(3)$ & $-11(3)$ & $3(3)$ & $-14(3)$ \\
\hline$C(40)$ & $50(5)$ & $47(5)$ & $44(4)$ & $11(4)$ & $-16(4)$ & $-24(4)$ \\
\hline$C(41)$ & $48(5)$ & $56(5)$ & $45(5)$ & $2(4)$ & $-10(4)$ & $-23(4)$ \\
\hline$C(42)$ & $58(6)$ & $40(5)$ & $23(4)$ & $-4(3)$ & $1(4)$ & $-25(4)$ \\
\hline$C(43)$ & $55(6)$ & $44(5)$ & $35(4)$ & $8(4)$ & $-13(4)$ & $-22(4)$ \\
\hline$C(44)$ & $51(5)$ & $56(5)$ & $34(4)$ & $10(4)$ & $-8(4)$ & $-31(4)$ \\
\hline$C(45)$ & $51(5)$ & $49(5)$ & $25(4)$ & $-6(4)$ & $-11(4)$ & $-18(5)$ \\
\hline$C(46)$ & $64(6)$ & $67(6)$ & $25(4)$ & $-1(4)$ & $-7(4)$ & $-38(5)$ \\
\hline$C(1 S)$ & $71(7)$ & $62(6)$ & $45(5)$ & $-10(4)$ & $0(5)$ & $-16(5)$ \\
\hline$C(2 S)$ & $78(7)$ & $83(7)$ & $48(5)$ & $9(5)$ & $-24(5)$ & $-32(6)$ \\
\hline $\mathrm{C}(3 \mathrm{~S})$ & $78(7)$ & $73(6)$ & $32(4)$ & $-1(4)$ & $-6(4)$ & $-31(6)$ \\
\hline$C(4 S)$ & $59(6)$ & $69(6)$ & $39(5)$ & $3(4)$ & $-7(4)$ & $-22(5)$ \\
\hline$C(5 S)$ & $70(7)$ & $76(7)$ & $54(6)$ & $1(5)$ & $-4(5)$ & $-33(6)$ \\
\hline$C(6 S)$ & $66(7)$ & $72(7)$ & $58(6)$ & $-10(5)$ & $-10(5)$ & $-27(5)$ \\
\hline
\end{tabular}


Table S15. Hydrogen coordinates and isotropic displacement parameters for 2.

\begin{tabular}{|c|c|c|c|c|}
\hline & $x$ & $y$ & z & $U(e q)$ \\
\hline$H(2)$ & 0.2357 & 0.1608 & 1.0119 & 0.041 \\
\hline $\mathrm{H}(3)$ & 0.2002 & 0.3524 & 1.0431 & 0.042 \\
\hline $\mathrm{H}(7)$ & 0.3661 & 0.6522 & 0.8768 & 0.043 \\
\hline$H(8)$ & 0.5146 & 0.6579 & 0.7339 & 0.044 \\
\hline $\mathrm{H}(12)$ & 0.7824 & 0.3811 & 0.4697 & 0.040 \\
\hline$H(13)$ & 0.8118 & 0.1924 & 0.4374 & 0.041 \\
\hline $\mathrm{H}(17)$ & 0.6427 & -0.1059 & 0.6047 & 0.043 \\
\hline $\mathrm{H}(18)$ & 0.5043 & -0.1153 & 0.7483 & 0.041 \\
\hline $\mathrm{H}(22)$ & 0.0843 & 0.6179 & 0.9557 & 0.052 \\
\hline $\mathrm{H}(23)$ & -0.0378 & 0.7322 & 1.0785 & 0.057 \\
\hline $\mathrm{H}(24)$ & 0.0643 & 0.7073 & 1.1839 & 0.052 \\
\hline$H(25)$ & 0.2875 & 0.5732 & 1.1682 & 0.051 \\
\hline $\mathrm{H}(26)$ & 0.4094 & 0.4595 & 1.0454 & 0.048 \\
\hline $\mathrm{H}(28)$ & 0.5138 & 0.6191 & 0.5339 & 0.054 \\
\hline $\mathrm{H}(29)$ & 0.5881 & 0.7533 & 0.4636 & 0.054 \\
\hline $\mathrm{H}(30)$ & 0.7900 & 0.7606 & 0.4701 & 0.057 \\
\hline $\mathrm{H}(31)$ & 0.9152 & 0.6384 & 0.5516 & 0.067 \\
\hline $\mathrm{H}(32)$ & 0.8423 & 0.5047 & 0.6223 & 0.061 \\
\hline $\mathrm{H}(34)$ & 0.6005 & 0.0651 & 0.4411 & 0.050 \\
\hline$H(35)$ & 0.7234 & -0.0502 & 0.3207 & 0.055 \\
\hline $\mathrm{H}(36)$ & 0.9538 & -0.1717 & 0.3010 & 0.049 \\
\hline $\mathrm{H}(37)$ & 1.0633 & -0.1800 & 0.4007 & 0.049 \\
\hline $\mathrm{H}(38)$ & 0.9372 & -0.0657 & 0.5225 & 0.049 \\
\hline $\mathrm{H}(40)$ & 0.1743 & 0.0404 & 0.8750 & 0.054 \\
\hline $\mathrm{H}(41)$ & 0.1060 & -0.0932 & 0.9529 & 0.060 \\
\hline $\mathrm{H}(42)$ & 0.2471 & -0.2200 & 1.0250 & 0.049 \\
\hline $\mathrm{H}(43)$ & 0.4598 & -0.2194 & 1.0158 & 0.053 \\
\hline $\mathrm{H}(44)$ & 0.5292 & -0.0872 & 0.9389 & 0.054 \\
\hline $\mathrm{H}(1 \mathrm{~S} 1)$ & 0.8245 & 0.9736 & 0.7606 & 0.080 \\
\hline $\mathrm{H}(1 \mathrm{~S} 2)$ & 0.7501 & 0.9627 & 0.8562 & 0.080 \\
\hline $\mathrm{H}(2 \mathrm{~S} 1)$ & 0.7535 & 0.8261 & 0.7714 & 0.082 \\
\hline $\mathrm{H}(2 \mathrm{~S} 2)$ & 0.8385 & 0.7674 & 0.8362 & 0.082 \\
\hline $\mathrm{H}(3 \mathrm{~S} 1)$ & 0.9751 & 0.6809 & 0.7013 & 0.076 \\
\hline $\mathrm{H}(3 \mathrm{~S} 2)$ & 0.9653 & 0.7991 & 0.6645 & 0.076 \\
\hline $\mathrm{H}(4 \mathrm{~S} 1)$ & 1.1045 & 0.6918 & 0.7852 & 0.071 \\
\hline $\mathrm{H}(4 \mathrm{~S} 2)$ & 1.1815 & 0.7027 & 0.6901 & 0.071 \\
\hline $\mathrm{H}(5 \mathrm{~S} 1)$ & 1.0981 & 0.8951 & 0.7096 & 0.082 \\
\hline $\mathrm{H}(5 \mathrm{~S} 2)$ & 1.1754 & 0.8368 & 0.7777 & 0.082 \\
\hline $\mathrm{H}(6 \mathrm{~S} 1)$ & 0.9611 & 0.8741 & 0.8814 & 0.080 \\
\hline
\end{tabular}


$\mathrm{H}(6 \mathrm{~S} 2)$

0.9529

0.9889

0.8390

0.080 
Table S16. Torsion angles $\left[{ }^{\circ}\right]$ for 2.

\begin{tabular}{|c|c|c|c|}
\hline $\mathrm{N}(5)-\mathrm{Fe}(1)-\mathrm{O}(1)-\mathrm{C}(45)$ & $-147(2)$ & $\mathrm{N}(2)-\mathrm{Fe}(1)-\mathrm{N}(4)-\mathrm{C}(6)$ & $91(4)$ \\
\hline $\mathrm{N}(2)-\mathrm{Fe}(1)-\mathrm{O}(1)-\mathrm{C}(45)$ & $49.7(6)$ & $\mathrm{N}(1)-\mathrm{Fe}(1)-\mathrm{N}(4)-\mathrm{C}(6)$ & $0.2(6)$ \\
\hline $\mathrm{N}(1)-\mathrm{Fe}(1)-\mathrm{O}(1)-\mathrm{C}(45)$ & $-39.9(6)$ & $\mathrm{N}(3)-\mathrm{Fe}(1)-\mathrm{N}(4)-\mathrm{C}(6)$ & $176.6(6)$ \\
\hline $\mathrm{N}(3)-\mathrm{Fe}(1)-\mathrm{O}(1)-\mathrm{C}(45)$ & $139.6(6)$ & $\mathrm{O}(1)-\mathrm{Fe}(1)-\mathrm{N}(5)-\mathrm{O}(3)$ & $-12(9)$ \\
\hline $\mathrm{N}(4)-\mathrm{Fe}(1)-\mathrm{O}(1)-\mathrm{C}(45)$ & $-130.3(6)$ & $\mathrm{N}(2)-\mathrm{Fe}(1)-\mathrm{N}(5)-\mathrm{O}(3)$ & $151(8)$ \\
\hline $\mathrm{N}(5)-\mathrm{Fe}(1)-\mathrm{N}(1)-\mathrm{C}(1)$ & $-88.4(6)$ & $\mathrm{N}(1)-\mathrm{Fe}(1)-\mathrm{N}(5)-\mathrm{O}(3)$ & $-119(8)$ \\
\hline $\mathrm{O}(1)-\mathrm{Fe}(1)-\mathrm{N}(1)-\mathrm{C}(1)$ & $97.2(6)$ & $\mathrm{N}(3)-\mathrm{Fe}(1)-\mathrm{N}(5)-\mathrm{O}(3)$ & 61(8) \\
\hline $\mathrm{N}(2)-\mathrm{Fe}(1)-\mathrm{N}(1)-\mathrm{C}(1)$ & $4.9(6)$ & $\mathrm{N}(4)-\mathrm{Fe}(1)-\mathrm{N}(5)-\mathrm{O}(3)$ & $-29(8)$ \\
\hline $\mathrm{N}(3)-\mathrm{Fe}(1)-\mathrm{N}(1)-\mathrm{C}(1)$ & $89(4)$ & $C(4)-N(1)-C(1)-C(20)$ & $178.9(7)$ \\
\hline $\mathrm{N}(4)-\mathrm{Fe}(1)-\mathrm{N}(1)-\mathrm{C}(1)$ & $-178.5(6)$ & $\mathrm{Fe}(1)-\mathrm{N}(1)-\mathrm{C}(1)-\mathrm{C}(20)$ & $-2.9(10)$ \\
\hline $\mathrm{N}(5)-\mathrm{Fe}(1)-\mathrm{N}(1)-\mathrm{C}(4)$ & $89.4(6)$ & $\mathrm{C}(4)-\mathrm{N}(1)-\mathrm{C}(1)-\mathrm{C}(2)$ & $0.8(8)$ \\
\hline $\mathrm{O}(1)-\mathrm{Fe}(1)-\mathrm{N}(1)-\mathrm{C}(4)$ & $-85.0(5)$ & $\mathrm{Fe}(1)-\mathrm{N}(1)-\mathrm{C}(1)-\mathrm{C}(2)$ & $178.9(5)$ \\
\hline $\mathrm{N}(2)-\mathrm{Fe}(1)-\mathrm{N}(1)-\mathrm{C}(4)$ & $-177.3(5)$ & $N(1)-C(1)-C(2)-C(3)$ & $-0.9(8)$ \\
\hline $\mathrm{N}(3)-\mathrm{Fe}(1)-\mathrm{N}(1)-\mathrm{C}(4)$ & $-93(4)$ & $C(20)-C(1)-C(2)-C(3)$ & $-179.1(7)$ \\
\hline$N(4)-F e(1)-N(1)-C(4)$ & $-0.7(5)$ & $C(1)-C(2)-C(3)-C(4)$ & $0.6(8)$ \\
\hline $\mathrm{N}(5)-\mathrm{Fe}(1)-\mathrm{N}(2)-\mathrm{C}(19)$ & $86.7(6)$ & $\mathrm{C}(1)-\mathrm{N}(1)-\mathrm{C}(4)-\mathrm{C}(5)$ & $178.5(7)$ \\
\hline $\mathrm{O}(1)-\mathrm{Fe}(1)-\mathrm{N}(2)-\mathrm{C}(19)$ & $-95.1(6)$ & $\mathrm{Fe}(1)-\mathrm{N}(1)-\mathrm{C}(4)-\mathrm{C}(5)$ & $0.3(10)$ \\
\hline $\mathrm{N}(1)-\mathrm{Fe}(1)-\mathrm{N}(2)-\mathrm{C}(19)$ & $-5.5(6)$ & $C(1)-N(1)-C(4)-C(3)$ & $-0.4(7)$ \\
\hline $\mathrm{N}(3)-\mathrm{Fe}(1)-\mathrm{N}(2)-\mathrm{C}(19)$ & 178.1(6) & $\mathrm{Fe}(1)-\mathrm{N}(1)-\mathrm{C}(4)-\mathrm{C}(3)$ & $-178.6(4)$ \\
\hline $\mathrm{N}(4)-\mathrm{Fe}(1)-\mathrm{N}(2)-\mathrm{C}(19)$ & $-96(4)$ & $C(2)-C(3)-C(4)-C(5)$ & $-179.1(7)$ \\
\hline $\mathrm{N}(5)-\mathrm{Fe}(1)-\mathrm{N}(2)-\mathrm{C}(16)$ & $-87.8(6)$ & $C(2)-C(3)-C(4)-N(1)$ & $-0.1(8)$ \\
\hline $\mathrm{O}(1)-\mathrm{Fe}(1)-\mathrm{N}(2)-\mathrm{C}(16)$ & $90.4(6)$ & $N(1)-C(4)-C(5)-C(6)$ & $0.7(12)$ \\
\hline $\mathrm{N}(1)-\mathrm{Fe}(1)-\mathrm{N}(2)-\mathrm{C}(16)$ & $-180.0(6)$ & $C(3)-C(4)-C(5)-C(6)$ & $179.5(7)$ \\
\hline $\mathrm{N}(3)-\mathrm{Fe}(1)-\mathrm{N}(2)-\mathrm{C}(16)$ & $3.6(6)$ & $N(1)-C(4)-C(5)-C(21)$ & $-178.2(6)$ \\
\hline $\mathrm{N}(4)-\mathrm{Fe}(1)-\mathrm{N}(2)-\mathrm{C}(16)$ & $89(4)$ & $C(3)-C(4)-C(5)-C(21)$ & $0.6(10)$ \\
\hline$N(5)-F e(1)-N(3)-C(11)$ & $-86.9(6)$ & $\mathrm{C}(9)-\mathrm{N}(4)-\mathrm{C}(6)-\mathrm{C}(5)$ & $179.6(7)$ \\
\hline $\mathrm{O}(1)-\mathrm{Fe}(1)-\mathrm{N}(3)-\mathrm{C}(11)$ & $87.4(6)$ & $\mathrm{Fe}(1)-\mathrm{N}(4)-\mathrm{C}(6)-\mathrm{C}(5)$ & $0.6(10)$ \\
\hline $\mathrm{N}(2)-\mathrm{Fe}(1)-\mathrm{N}(3)-\mathrm{C}(11)$ & 179.7(6) & $\mathrm{C}(9)-\mathrm{N}(4)-\mathrm{C}(6)-\mathrm{C}(7)$ & $-0.6(8)$ \\
\hline $\mathrm{N}(1)-\mathrm{Fe}(1)-\mathrm{N}(3)-\mathrm{C}(11)$ & $96(4)$ & $\mathrm{Fe}(1)-\mathrm{N}(4)-\mathrm{C}(6)-\mathrm{C}(7)$ & $-179.6(5)$ \\
\hline $\mathrm{N}(4)-\mathrm{Fe}(1)-\mathrm{N}(3)-\mathrm{C}(11)$ & $3.1(6)$ & $C(4)-C(5)-C(6)-N(4)$ & $-1.3(12)$ \\
\hline$N(5)-F e(1)-N(3)-C(14)$ & $90.3(6)$ & $\mathrm{C}(21)-\mathrm{C}(5)-\mathrm{C}(6)-\mathrm{N}(4)$ & $177.7(6)$ \\
\hline $\mathrm{O}(1)-\mathrm{Fe}(1)-\mathrm{N}(3)-\mathrm{C}(14)$ & $-95.4(6)$ & $C(4)-C(5)-C(6)-C(7)$ & $179.0(7)$ \\
\hline $\mathrm{N}(2)-\mathrm{Fe}(1)-\mathrm{N}(3)-\mathrm{C}(14)$ & $-3.1(6)$ & $\mathrm{C}(21)-\mathrm{C}(5)-\mathrm{C}(6)-\mathrm{C}(7)$ & $-2.1(11)$ \\
\hline $\mathrm{N}(1)-\mathrm{Fe}(1)-\mathrm{N}(3)-\mathrm{C}(14)$ & $-87(4)$ & $N(4)-C(6)-C(7)-C(8)$ & $0.3(8)$ \\
\hline $\mathrm{N}(4)-\mathrm{Fe}(1)-\mathrm{N}(3)-\mathrm{C}(14)$ & $-179.7(6)$ & $C(5)-C(6)-C(7)-C(8)$ & $-179.9(7)$ \\
\hline $\mathrm{N}(5)-\mathrm{Fe}(1)-\mathrm{N}(4)-\mathrm{C}(9)$ & $89.3(6)$ & $\mathrm{C}(6)-\mathrm{C}(7)-\mathrm{C}(8)-\mathrm{C}(9)$ & $0.1(8)$ \\
\hline $\mathrm{O}(1)-\mathrm{Fe}(1)-\mathrm{N}(4)-\mathrm{C}(9)$ & $-89.0(6)$ & $\mathrm{C}(6)-\mathrm{N}(4)-\mathrm{C}(9)-\mathrm{C}(10)$ & $-178.6(7)$ \\
\hline $\mathrm{N}(2)-\mathrm{Fe}(1)-\mathrm{N}(4)-\mathrm{C}(9)$ & $-88(4)$ & $\mathrm{Fe}(1)-\mathrm{N}(4)-\mathrm{C}(9)-\mathrm{C}(10)$ & $0.4(10)$ \\
\hline $\mathrm{N}(1)-\mathrm{Fe}(1)-\mathrm{N}(4)-\mathrm{C}(9)$ & $-178.6(6)$ & $\mathrm{C}(6)-\mathrm{N}(4)-\mathrm{C}(9)-\mathrm{C}(8)$ & $0.6(8)$ \\
\hline $\mathrm{N}(3)-\mathrm{Fe}(1)-\mathrm{N}(4)-\mathrm{C}(9)$ & $-2.2(6)$ & $\mathrm{Fe}(1)-\mathrm{N}(4)-\mathrm{C}(9)-\mathrm{C}(8)$ & 179.6(5) \\
\hline $\mathrm{N}(5)-\mathrm{Fe}(1)-\mathrm{N}(4)-\mathrm{C}(6)$ & $-92.0(6)$ & $\mathrm{C}(7)-\mathrm{C}(8)-\mathrm{C}(9)-\mathrm{N}(4)$ & $-0.4(8)$ \\
\hline $\mathrm{O}(1)-\mathrm{Fe}(1)-\mathrm{N}(4)-\mathrm{C}(6)$ & $89.8(6)$ & $C(7)-C(8)-C(9)-C(10)$ & $178.8(7)$ \\
\hline
\end{tabular}




\begin{tabular}{|c|c|c|c|}
\hline$N(4)-C(9)-C(10)-C(11)$ & $1.6(12)$ & $C(2)-C(1)-C(20)-C(39)$ & $-2.4(10)$ \\
\hline$C(8)-C(9)-C(10)-C(11)$ & $-177.6(7)$ & $N(2)-C(19)-C(20)-C(1)$ & $0.1(11)$ \\
\hline $\mathrm{N}(4)-\mathrm{C}(9)-\mathrm{C}(10)-\mathrm{C}(27)$ & $-179.1(7)$ & $C(18)-C(19)-C(20)-C(1)$ & $-178.5(7)$ \\
\hline$C(8)-C(9)-C(10)-C(27)$ & $1.8(10)$ & $\mathrm{N}(2)-\mathrm{C}(19)-\mathrm{C}(20)-\mathrm{C}(39)$ & $179.7(6)$ \\
\hline $\mathrm{C}(14)-\mathrm{N}(3)-\mathrm{C}(11)-\mathrm{C}(10)$ & $180.0(7)$ & $C(18)-C(19)-C(20)-C(39)$ & $1.1(10)$ \\
\hline $\mathrm{Fe}(1)-\mathrm{N}(3)-\mathrm{C}(11)-\mathrm{C}(10)$ & $-2.4(10)$ & $C(4)-C(5)-C(21)-C(22)$ & $-95.7(8)$ \\
\hline $\mathrm{C}(14)-\mathrm{N}(3)-\mathrm{C}(11)-\mathrm{C}(12)$ & $0.7(8)$ & $C(6)-C(5)-C(21)-C(22)$ & $85.3(9)$ \\
\hline $\mathrm{Fe}(1)-\mathrm{N}(3)-\mathrm{C}(11)-\mathrm{C}(12)$ & $178.3(5)$ & $C(4)-C(5)-C(21)-C(26)$ & $79.8(9)$ \\
\hline$C(9)-C(10)-C(11)-N(3)$ & $-0.5(12)$ & $C(6)-C(5)-C(21)-C(26)$ & $-99.2(8)$ \\
\hline $\mathrm{C}(27)-\mathrm{C}(10)-\mathrm{C}(11)-\mathrm{N}(3)$ & $-179.8(7)$ & $C(26)-C(21)-C(22)-C(23)$ & $0.7(11)$ \\
\hline$C(9)-C(10)-C(11)-C(12)$ & $178.7(7)$ & $C(5)-C(21)-C(22)-C(23)$ & $176.3(7)$ \\
\hline$C(27)-C(10)-C(11)-C(12)$ & $-0.6(11)$ & $C(21)-C(22)-C(23)-C(24)$ & $-0.7(12)$ \\
\hline$N(3)-C(11)-C(12)-C(13)$ & $-0.4(8)$ & $C(22)-C(23)-C(24)-C(25)$ & $0.6(12)$ \\
\hline$C(10)-C(11)-C(12)-C(13)$ & $-179.7(7)$ & $C(23)-C(24)-C(25)-C(26)$ & $-0.5(11)$ \\
\hline$C(11)-C(12)-C(13)-C(14)$ & $-0.1(8)$ & $C(22)-C(21)-C(26)-C(25)$ & $-0.6(11)$ \\
\hline$C(11)-N(3)-C(14)-C(15)$ & $179.2(7)$ & $C(5)-C(21)-C(26)-C(25)$ & $-176.3(7)$ \\
\hline $\mathrm{Fe}(1)-\mathrm{N}(3)-\mathrm{C}(14)-\mathrm{C}(15)$ & $1.6(10)$ & $C(24)-C(25)-C(26)-C(21)$ & $0.5(11)$ \\
\hline$C(11)-N(3)-C(14)-C(13)$ & $-0.7(8)$ & $C(11)-C(10)-C(27)-C(28)$ & $85.6(8)$ \\
\hline $\mathrm{Fe}(1)-\mathrm{N}(3)-\mathrm{C}(14)-\mathrm{C}(13)$ & $-178.4(5)$ & $C(9)-C(10)-C(27)-C(28)$ & $-93.8(8)$ \\
\hline$C(12)-C(13)-C(14)-N(3)$ & $0.5(8)$ & $C(11)-C(10)-C(27)-C(32)$ & $-93.6(8)$ \\
\hline$C(12)-C(13)-C(14)-C(15)$ & $-179.5(7)$ & $C(9)-C(10)-C(27)-C(32)$ & $87.0(9)$ \\
\hline$N(3)-C(14)-C(15)-C(16)$ & $0.8(12)$ & $C(32)-C(27)-C(28)-C(29)$ & $-1.3(10)$ \\
\hline$C(13)-C(14)-C(15)-C(16)$ & $-179.2(7)$ & $C(10)-C(27)-C(28)-C(29)$ & $179.5(6)$ \\
\hline$N(3)-C(14)-C(15)-C(33)$ & $-179.6(7)$ & $\mathrm{C}(27)-\mathrm{C}(28)-\mathrm{C}(29)-\mathrm{C}(30)$ & $1.3(11)$ \\
\hline$C(13)-C(14)-C(15)-C(33)$ & $0.4(11)$ & $C(28)-C(29)-C(30)-C(31)$ & $-1.1(11)$ \\
\hline$C(19)-N(2)-C(16)-C(15)$ & $-178.1(7)$ & $C(29)-C(30)-C(31)-C(32)$ & $1.0(12)$ \\
\hline $\mathrm{Fe}(1)-\mathrm{N}(2)-\mathrm{C}(16)-\mathrm{C}(15)$ & $-2.7(10)$ & $\mathrm{C}(28)-\mathrm{C}(27)-\mathrm{C}(32)-\mathrm{C}(31)$ & $1.1(11)$ \\
\hline$C(19)-N(2)-C(16)-C(17)$ & $2.0(8)$ & $C(10)-C(27)-C(32)-C(31)$ & $-179.6(7)$ \\
\hline $\mathrm{Fe}(1)-\mathrm{N}(2)-\mathrm{C}(16)-\mathrm{C}(17)$ & $177.4(5)$ & $C(30)-C(31)-C(32)-C(27)$ & $-1.0(12)$ \\
\hline$C(14)-C(15)-C(16)-N(2)$ & $-0.3(12)$ & $C(14)-C(15)-C(33)-C(38)$ & $91.2(8)$ \\
\hline$C(33)-C(15)-C(16)-N(2)$ & $-179.8(7)$ & $C(16)-C(15)-C(33)-C(38)$ & $-89.2(9)$ \\
\hline$C(14)-C(15)-C(16)-C(17)$ & $179.6(7)$ & $C(14)-C(15)-C(33)-C(34)$ & $-87.8(9)$ \\
\hline$C(33)-C(15)-C(16)-C(17)$ & $0.1(11)$ & $C(16)-C(15)-C(33)-C(34)$ & $91.8(9)$ \\
\hline $\mathrm{N}(2)-\mathrm{C}(16)-\mathrm{C}(17)-\mathrm{C}(18)$ & $-1.5(8)$ & $C(38)-C(33)-C(34)-C(35)$ & $-2.1(11)$ \\
\hline$C(15)-C(16)-C(17)-C(18)$ & $178.5(7)$ & $C(15)-C(33)-C(34)-C(35)$ & $176.9(7)$ \\
\hline$C(16)-C(17)-C(18)-C(19)$ & $0.5(8)$ & $C(33)-C(34)-C(35)-C(36)$ & $0.6(11)$ \\
\hline$C(16)-N(2)-C(19)-C(20)$ & $179.6(7)$ & $C(34)-C(35)-C(36)-C(37)$ & $0.4(11)$ \\
\hline $\mathrm{Fe}(1)-\mathrm{N}(2)-\mathrm{C}(19)-\mathrm{C}(20)$ & $4.2(10)$ & $C(35)-C(36)-C(37)-C(38)$ & $0.2(11)$ \\
\hline $\mathrm{C}(16)-\mathrm{N}(2)-\mathrm{C}(19)-\mathrm{C}(18)$ & $-1.7(8)$ & $C(34)-C(33)-C(38)-C(37)$ & $2.7(10)$ \\
\hline $\mathrm{Fe}(1)-\mathrm{N}(2)-\mathrm{C}(19)-\mathrm{C}(18)$ & $-177.1(5)$ & $C(15)-C(33)-C(38)-C(37)$ & $-176.3(7)$ \\
\hline$C(17)-C(18)-C(19)-N(2)$ & $0.8(8)$ & $C(36)-C(37)-C(38)-C(33)$ & $-1.8(11)$ \\
\hline$C(17)-C(18)-C(19)-C(20)$ & $179.5(7)$ & $C(1)-C(20)-C(39)-C(40)$ & $86.5(8)$ \\
\hline$N(1)-C(1)-C(20)-C(19)$ & $-0.7(12)$ & $C(19)-C(20)-C(39)-C(40)$ & $-93.1(8)$ \\
\hline$C(2)-C(1)-C(20)-C(19)$ & $177.2(7)$ & $C(1)-C(20)-C(39)-C(44)$ & $-90.9(8)$ \\
\hline$N(1)-C(1)-C(20)-C(39)$ & $179.7(6)$ & $C(19)-C(20)-C(39)-C(44)$ & $89.5(8)$ \\
\hline
\end{tabular}




\begin{tabular}{|c|c|c|c|}
\hline$C(44)-C(39)-C(40)-C(41)$ & $-0.4(10)$ & $\mathrm{O}(1)-\mathrm{C}(45)-\mathrm{C}(46)-\mathrm{F}(3)$ & $153.0(6)$ \\
\hline$C(20)-C(39)-C(40)-C(41)$ & $-177.8(6)$ & $\mathrm{O}(2)-\mathrm{C}(45)-\mathrm{C}(46)-\mathrm{F}(2)$ & $-153.8(6)$ \\
\hline$C(39)-C(40)-C(41)-C(42)$ & $0.8(11)$ & $\mathrm{O}(1)-\mathrm{C}(45)-\mathrm{C}(46)-\mathrm{F}(2)$ & 30.6(9) \\
\hline$C(40)-C(41)-C(42)-C(43)$ & $-1.2(11)$ & $\mathrm{O}(2)-\mathrm{C}(45)-\mathrm{C}(46)-\mathrm{F}(1)$ & 86.7(8) \\
\hline$C(41)-C(42)-C(43)-C(44)$ & $1.3(11)$ & $\mathrm{O}(1)-\mathrm{C}(45)-\mathrm{C}(46)-\mathrm{F}(1)$ & $-88.9(7)$ \\
\hline$C(40)-C(39)-C(44)-C(43)$ & $0.5(10)$ & $C(6 S)-C(1 S)-C(2 S)-C(3 S)$ & $-57.2(10)$ \\
\hline$C(20)-C(39)-C(44)-C(43)$ & $177.9(6)$ & $C(1 S)-C(2 S)-C(3 S)-C(4 S)$ & $57.4(10)$ \\
\hline$C(42)-C(43)-C(44)-C(39)$ & $-0.9(11)$ & $C(2 S)-C(3 S)-C(4 S)-C(5 S)$ & $-58.4(10)$ \\
\hline $\mathrm{Fe}(1)-\mathrm{O}(1)-\mathrm{C}(45)-\mathrm{O}(2)$ & $-17.5(11)$ & $C(3 S)-C(4 S)-C(5 S)-C(6 S)$ & $55.2(10)$ \\
\hline $\mathrm{Fe}(1)-\mathrm{O}(1)-\mathrm{C}(45)-\mathrm{C}(46)$ & $157.6(5)$ & $C(2 S)-C(1 S)-C(6 S)-C(5 S)$ & $55.3(11)$ \\
\hline $\mathrm{O}(2)-\mathrm{C}(45)-\mathrm{C}(46)-\mathrm{F}(3)$ & $-31.4(9)$ & $C(4 S)-C(5 S)-C(6 S)-C(1 S)$ & $-53.2(11)$ \\
\hline
\end{tabular}

